\title{
The tunnel magneto-Seebeck effect in magnetic tunnel junctions
}

\section{Dissertation}

zur Erlangung des mathematisch-naturwissenschaftlichen Doktorgrades

"Doctor rerum naturalium"

der Georg-August-Universität Göttingen

im Promotionsprogramm ProPhys

der Georg-August University School of Science (GAUSS)

vorgelegt von

\section{Marvin Walter}

aus Göttingen

Göttingen, 2013 


\section{Betreuungsausschuss:}

Prof. Dr. Markus Münzenberg, I. Physikalisches Institut

Prof. Dr. Christian Jooß, Institut für Materialphysik

Mitglieder der Prüfungskommission:

Referent: Prof. Dr. Markus Münzenberg, I. Physikalisches Institut

Korreferent: Prof. Dr. Christian Jooß, Institut für Materialphysik

weitere Mitglieder der Prüfungskommission:

Prof. Dr. Konrad Samwer, I. Physikalisches Institut

Prof. Dr. Hans-Ulrich Krebs, Institut für Materialphysik

Prof. Dr. Thomas Pruschke, Institut für Theoretische Physik

Prof. Dr. Angela Rizzi, IV. Physikalisches Institut

Tag der mündlichen Prüfung: 14. November 2013 


\section{Contents}

1 Introduction $\quad \mathbf{1}$

1.1 Thermodynamical kinetic equations and the thermoelectric effects . 3

1.1.1 The Seebeck effect . . . . . . . . . . . . . . 4

1.1.2 The Peltier effect . . . . . . . . . . . . . 6

1.1.3 The Thomson effect. . . . . . . . . . . . . 7

1.1.4 Thermomagnetic effects . . . . . . . . . . . . 7

1.1.5 Spin-dependent effects in metallic magnetic multilayers . . . 9

1.2 The spin Seebeck effect . . . . . . . . . . . . . . . . . . 10

1.3 Spin-dependent Seebeck and Peltier effects in spin valves . . . . . . 12

1.4 Spin-dependent Seebeck effects in tunneling systems . . . . . . . . . 14

1.4.1 Seebeck spin tunneling into silicon . . . . . . . . . . . . . . . 14

1.4.2 The tunnel magneto-Seebeck effect . . . . . . . . . . . . . 16

1.4.3 Thermal spin-transfer torque . . . . . . . . . . . . 19

2 Seebeck effect in magnetic tunnel junctions 23

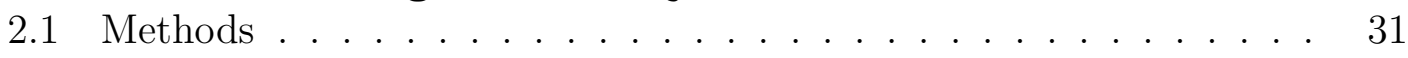

2.1.1 Fabrication ...................... 31

2.1.2 Experimental set-up . . . . . . . . . . . . . . 32

2.1.3 Thermal modelling of parameters (COMSOL) . . . . . . . 32

2.2 Acknowledgements . . . . . . . . . . . . . . . . . . . . . . 33

2.3 Author contributions . . . . . . . . . . . . . . . . . . . . 33

2.4 Additional information . . . . . . . . . . . . . . . . . 33

3 Time-resolved measurement of the tunnel magneto-Seebeck effect 35

3.1 Introduction . . . . . . . . . . . . . . . . . . . 35

3.2 Description of the experimental setup . . . . . . . . . . . . . . . . . 36

3.2.1 Determination of temperatures . . . . . . . . . . . . 38

3.3 Sample preparation . . . . . . . . . . . . . . . . 39

3.4 Experiments on magnesium oxide and silicon substrates . . . . . . . 39

3.4.1 TMR and TMS measurements . . . . . . . . . . . . . . . . 39

3.4.2 Thermocurrent measurements . . . . . . . . . . . . . . . 42

3.4.3 Time-dependent thermovoltage signals . . . . . . . . . . 43

3.5 Development of a model circuit . . . . . . . . . . . . . . . . . . . . 44

3.6 Position-dependent measurements . . . . . . . . . . . . . . . . 47

3.7 Conclusion . . . . . . . . . . . . . . . . . . . . . . 47 
3.8 Acknowledgments . . . . . . . . . . . . . . . . . . . . . . . . 48

4 Parameter space for thermal spin transfer torque $\quad 49$

4.1 Introduction . . . . . . . . . . . . . . . . . . . . . 49

4.2 Sample Preparation . . . . . . . . . . . . . . . . . 50

4.3 Growth of Ultra Thin Barriers . . . . . . . . . . . . . . . . . . 50

4.4 Quantitative HRTEM Analysis of MgO Barriers . . . . . . . . . . 51

4.5 Spin-transfer Torque in PMA Junctions . . . . . . . . . . . . . . . 53

4.6 Torque Simulations . . . . . . . . . . . . . . . . . . . . 54

4.7 Temperature Simulations . . . . . . . . . . . . . . . . . . . . . . . . 55

4.8 Conclusion . . . . . . . . . . . . . . . . . . . . . . . 57

4.9 Acknowledgments . . . . . . . . . . . . . . . . . 57

5 Outlook $\mathbf{5 9}$

5.1 Bias voltage dependence of the tunnel magneto-Seebeck effect . . . 59

5.1.1 Sample preparation . . . . . . . . . . . . . . 61

5.1 .2 Experimental setup . . . . . . . . . . . . . . 62

5.1.3 Estimate of Peltier and Thomson effects . . . . . . . . . 63

5.1 .4 First results . . . . . . . . . . . . . . . . . . . 64

5.1 .5 Discussion . . . . . . . . . . . . . . . . 67

5.2 Tunnel magneto-Seebeck effect in Heusler compound tunnel junctions 68

5.2.1 Sample preparation and TMR . . . . . . . . . . 70

5.2.2 Frequency dependence of the Seebeck voltage . . . . . . . 71

5.2 .3 Dependence on applied laser power . . . . . . . . . . . 73

5.2.4 Thermocurrent measurements . . . . . . . . . . . . 75

5.2.5 Position-dependent measurements . . . . . . . . . . 75

5.2 .6 Discussion . . . . . . . . . . . . . . . . . . . 78

5.3 The thermoelectric figure of merit . . . . . . . . . . . . 79

6 Summary $\quad 85$

A Supplementary information of Nature Materials 10, 742 (2011) $\quad 89$

A.1 Details of the laser heating and Seebeck voltage transients . . . . . 89

A.2 Lateral heating in the Au bond pad . . . . . . . . . . . . . . 91

A.3 Fluence dependence of the Seebeck voltages . . . . . . . . . . . . 92

$\begin{array}{ll}\text { Bibliography } & 95\end{array}$

$\begin{array}{ll}\text { Author contributions } & 103\end{array}$

$\begin{array}{lr}\text { Acknowledgements } & 105\end{array}$ 


\section{List of Figures}

1.1 Measurement of the Seebeck effect . . . . . . . . . . . . . . . 5

1.2 Geometries for thermomagnetic effects . . . . . . . . . . . . 8

1.3 The spin Seebeck effect . . . . . . . . . . . . . . . . . . . 11

1.4 Device for detection of the spin-dependent Seebeck effect . . . . . . 12

1.5 Direct measurement of the spin-dependent Peltier effect . . . . . . . 13

1.6 Seebeck spin tunneling into silicon . . . . . . . . . . . . . . . . . . 15

1.7 Tunnel magneto-Seebeck effect in alumina junctions . . . . . . . . . 17

1.8 Resistive heating of a $\mathrm{Co}-\mathrm{Fe}-\mathrm{B} / \mathrm{MgO}$ tunnel junction . . . . . . . . 18

1.9 Resistive heating: Seebeck voltages and thermocurrents . . . . . . . 19

1.10 Angular dependence of thermal spin-transfer torque . . . . . . . . . 20

2.1 Origin of the magneto-Seebeck effect . . . . . . . . . . . . . 25

2.2 Switching of the Seebeck effect through the magnetization . . . . . 28

2.3 Cross-sections and temperature gradients in the tunnel junction . . 29

2.4 Seebeck voltages for Fe-Co-B/MgO/Fe-Co-B elements . . . . . . . . 30

3.1 Experimental setup for measurements of the TMS effect . . . . . . 37

3.2 Low laser power and different substrates . . . . . . . . . . . . . 41

3.3 High laser power: Seebeck voltage and Seebeck current . . . . . . . 43

3.4 Time-dependent voltage signals of MTJs . . . . . . . . . . . . . . . 44

3.5 Model circuit for MTJs . . . . . . . . . . . . . . . . . . . . . . 45

3.6 Position-dependent measurements . . . . . . . . . . . . . . . . 48

4.1 HRTEM images of a $10 \mathrm{ML}$ and $3 \mathrm{ML}$ MgO barrier . . . . . . . . . 51

4.2 Quantitative analysis of the HR-TEM images . . . . . . . . . . . . 52

4.3 Electrical characterization of an MTJ . . . . . . . . . . . . 53

4.4 Calculated thermal torque at different lead temperatures . . . . . 55

4.5 Temperature simulations . . . . . . . . . . . . . . 56

5.1 Ab initio calculations of Seebeck coefficients . . . . . . . . . . . 60

5.2 Temperature dependence of Seebeck coefficients . . . . . . . . . . . 61

5.3 Experimental setup for TMS measurements with bias voltage . . . . 62

5.4 Dependence of TMR ratio on bias voltage . . . . . . . . . . . 64

5.5 Thermocurrent measurements at different bias voltages . . . . . . . 65

5.6 Bias voltage dependence of the TMS effect . . . . . . . . . . . . 67

5.7 Spin polarization of half metals . . . . . . . . . . . . . . . . 69 
5.8 Heusler compound sample: layer stack and TMR . . . . . . . . . . 70

5.9 Frequency dependence of the Seebeck voltage . . . . . . . . . . . 71

5.10 Dependence on applied laser power . . . . . . . . . . . . . . . . 74

5.11 Thermocurrent measurements of Heusler compound MTJs . . . . . 76

5.12 Position-dependent measurements of Heusler compound MTJs . . . 77

5.13 Electrical and thermal conductivities of MTJs . . . . . . . . . . . 81

5.14 Thermoelectric figure of merit of MTJs . . . . . . . . . . . . . . 82

A.1 COMSOL simulation on the larger length scale . . . . . . . . . . . . 91

A.2 Seebeck voltage for a tunnel junction heated with low laser fluencies 92

A.3 Seebeck voltage for a tunnel junction heated with high laser fluences 93 


\section{List of Tables}

2.1 Seebeck coefficients and magneto-Seebeck effects for different supercells 27

3.1 Material parameters for COMSOL simulations . . . . . . . . . . 38

3.2 Comparison of TMR and TMS on $\mathrm{MgO}$ and $\mathrm{Si} / \mathrm{SiO}_{2}$ samples . . . . 42

3.3 Basic estimations for the model circuit . . . . . . . . . . . . . . 46

A.1 Material parameters for COMSOL simulations . . . . . . . . . . 90 



\section{Chapter 1}

\section{Introduction}

The discoveries of thermoelectric effects date back to the beginning of the $19^{\text {th }}$ century. In 1821 Thomas Johann Seebeck observed an electrical current flowing through two interconnected, disparate metal plates when a temperature difference was applied across the junction ${ }^{1}$. This effect is now referred to as the Seebeck effect. A few years later, in 1834, Jean Charles Athanase Peltier was the first to measure the heat released or absorbed in such a junction when an electrical current is driven through the junction under isothermal conditions ${ }^{2}$, now known as the Peltier effect. A strong collaboration between James Prescott Joule and William Thomson led amongst other things to the prediction and discovery of a third thermoelectric effect, the Thomson effect, around 1854. It was found that any conductor is heated by an electrical current (Joule heating) and that, in the case of a current flowing in the presence of a temperature gradient, another heat quantity (Thomson heat) appears, which can be reversed by the current direction. William Thomson was then the first to develop a theory that explains these findings and reveals the relations between these three thermoelectric effects ${ }^{3}$.

Today, the Seebeck effect is commonly used in electrical thermometers and the Peltier effect is exploited to construct thermoelectric heating or cooling devices. Usually, Peltier elements are only used under special circumstances, because of their poor thermodynamic efficiency. Since the Peltier elements are very scalable in size, they are often deployed when a very small cooling device footprint is needed. As a consequence, there is still extensive on-going research with the aim of finding suitable materials to build efficient thermoelectric devices.

A first conjunction between thermoelectric effects and spin-dependent transport in ferromagnetic metals and across material interfaces was made in the groundbreaking theoretical work of M. Johnson and R. H. Silsbee ${ }^{4}$. But only a decade ago the research field known as spin caloritronics ${ }^{5}$ emerged, after the pioneering work of Parkin's ${ }^{6}$ and Ansermet's groups ${ }^{7-9}$ on thermoelectric effects in Giant Magnetoresistance (GMR) multilayers, and ultimately with the discovery of a new phenomenon, the spin-Seebeck effect, by Uchida et al. ${ }^{10}$ in 2008. This new research field has recently attracted considerable attention, driven not only by the prospects for fundamental research to observe theoretically predicted effects or even discover 
new spin-related phenomena, but also by the need for new strategies and devices in information technology. The continuous down-scaling of todays' complementary metal-oxide-semiconductor (CMOS) logic devices is reaching a point at which the energy dissipation due to ohmic losses in the devices is no longer bearable ${ }^{11}$. Spintronic devices, especially magnetic tunnel junctions - which are the focus of this thesis - open up various interesting approaches to overcome this issue, while offering writing speed and scalability comparable to, and allowing integration with, actual CMOS logic ${ }^{12-14}$.

This thesis is embedded in the broad and active fields of spintronics and spin caloritronics. The work presented in the following chapters focusses on the socalled tunnel magneto-Seebeck effect in magnetic tunnel junctions based on the $\mathrm{Co}-\mathrm{Fe}-\mathrm{B} / \mathrm{MgO}$ layer stack. Apart from several open questions for fundamental research, this material system is technologically relevant, due to the huge tunnel magnetoresistance effect of up to $600 \%$ at room temperature ${ }^{15}$ and the possibility of industrial-scale production by standard DC- and RF-sputtering techniques ${ }^{16}$.

The outline of the thesis is as follows: In chapter 1, section 1.1, the derivation of the thermodynamic kinetic equations is sketched and the basic three thermoelectric effects are discussed. Subsequently, a short introduction to the extension of these equations in the presence of an external magnetic field or for a ferromagnetic material is given. The relations introduced in this section are used throughout this thesis. The remainder of the chapter then presents a selection of recent reports on spin caloritronic effects that are related to this thesis: Section 1.2 gives a short overview of the spin-Seebeck effect, followed by an introduction to spin-dependent thermoelectric effects in metallic spin valves (section 1.3) and in tunnel junctions (section 1.4).

Chapters 2-4 contain reprints of three peer-reviewed articles that represent the main part of this thesis. In chapter 2 , ab initio calculations of the tunnel magnetoSeebeck effect in a $\mathrm{Co}-\mathrm{Fe} / \mathrm{MgO}$ tunnel junction are presented in combination with the first experimental observations of this theoretically predicted effect, which were achieved by optical heating of the tunnel junctions. Chapter 3 is devoted to the detailed analysis of the time-dependent voltage signals that were already shown in chapter 2. This analysis is made possible by experimental efforts in improving the setup with respect to time-resolution and signal-to-noise ratio. The effects in magnetic tunnel junctions grown on different substrates are studied and it is shown that the detected Seebeck voltages are locally generated in the tunnel junction. In chapter 4 , the parameter space for another, theoretically predicted effect, the thermal spin-transfer torque, is investigated by means of the optimization of growth conditions and the related electrical characterization of the tunnel junctions, as well as $a b$ initio calculations of the thermal torques and finite-elements simulations of the heat transport in the tunnel junction.

Chapter 5 contains first results of the two most recent experiment series and an estimate of the thermoelectric figure of merit for the magnetic tunnel junctions 
used in this thesis. The presentation and discussion of this unpublished data is meant to demonstrate possibilities for future theses and research. The discussion also points out the requirements for further theoretical work and the potential of magnetic tunnel junctions as efficient thermoelectric devices. Finally, in chapter 6 , the work presented in the thesis at hand is summarized.

A statement on author contributions to the reprinted publications, as required by the PhD program regulations of the Georg-August University School of Science ${ }^{17}$, can be found behind the bibliography.

\subsection{Thermodynamical kinetic equations and the thermoelectric effects}

The thermoelectric effects, which are topic of this thesis, can be described by the thermodynamic kinetic equations. These equations can be derived from thermodynamic principles by starting with the total derivative of the entropy per unit volume $s^{18}$ :

$$
\mathrm{d} s=\frac{1}{T} \mathrm{~d} u-\frac{\mu}{T} \mathrm{~d} n,
$$

in which $T$ is the temperature, $\mu$ is the electrochemical potential for the electrons, $u$ is the internal energy per unit volume, and $n$ is the number of electrons per unit volume. Contributions from other parts of the solid have been neglected. From eq. (1.1) follows that the entropy current density $\mathbf{J}_{S}$ can be written as

$$
\mathbf{J}_{S}=\frac{1}{T} \mathbf{J}_{U}-\frac{\mu}{T} \mathbf{J}_{N}
$$

The rate of entropy change with time $t, \dot{s}$, can thus be written $\mathrm{as}^{18}$ :

$$
\dot{s}=\frac{\partial s}{\partial t}+\nabla \cdot \mathbf{J}_{s}
$$

As described in detail in ref. [18], by applying continuity equations and the relationship between the heat $Q$ and the entropy $S(\mathrm{~d} Q=T \mathrm{~d} S)$, the rate of entropy change can be written in the form:

$$
\dot{s}=\mathbf{F}_{2} \cdot \mathbf{J}_{Q}-\mathbf{F}_{1} \mathbf{J}_{N}
$$

By using a linearization ansatz, it follows from this equation that the current densities $\mathbf{J}_{Q}$ and $\mathbf{J}_{N}$ can be expressed in terms of so-called generalized forces ${ }^{18} \mathbf{F}_{j}$ :

$$
-\mathbf{J}_{N}=\sum_{j} L_{1 j} \mathbf{F}_{j}, \quad \mathbf{J}_{Q}=\sum_{j} L_{2 j} \mathbf{F}_{j}
$$


If an electric current density $\mathbf{J}$ is considered at this point instead of the particle current density $\mathbf{J}_{N}$, and the electric potential $\Phi$ is used instead of the electrochemical potential, the kinetic equations become ${ }^{19,20}$ :

$$
\begin{gathered}
\mathbf{J}=L_{11}(-\nabla \Phi)+L_{12}(-\nabla T), \\
\mathbf{J}_{Q}=L_{21}(-\nabla \Phi)+L_{22}(-\nabla T) .
\end{gathered}
$$

An approach to these kinetic equations using the Boltzmann equation in the relaxation time approximation is pursued in refs. [19, 20]. The results lead to the following description of the kinetic coefficients ${ }^{19}$ :

$$
\begin{aligned}
& L_{11}=\mathcal{L}_{0}, \\
& L_{21}=T L_{12}=-\frac{1}{e} \mathcal{L}_{1}, \\
& L_{22}=\frac{1}{e^{2} T} \mathcal{L}_{2},
\end{aligned}
$$

in which the moments are given by:

$$
\mathcal{L}_{n}=\int \sigma(E)(E-\mu)^{n}\left(-\frac{\partial f}{\partial E}\right) \mathrm{d} E
$$

with the electrical conductivity $\sigma(E)$ at energy $E$ and the distribution function $f$.

The theoretical description of transport in tunnel junctions takes place in the framework of the Landauer formalism, in which transmission probabilities $\mathcal{T}(E)$ through the tunnel barrier are calculated. The moments are in this case given $\operatorname{as}^{21,22}$ :

$$
\mathcal{L}_{n}=\frac{2 e^{2}}{h} \int \mathcal{T}(E)(E-\mu)^{n}\left(-\frac{\partial f(E, \mu, T)}{\partial E}\right) \mathrm{d} E,
$$

in which the factor $e^{2}$ was added to the version found in ref. [22], to keep it consistent with the definitions of the coefficients (eqs. (1.5) to (1.7)).

The kinetic equations (1.3) and (1.4) with the coefficients (eqs. (1.5) to (1.7)) and moments (eqs. (1.8) or (1.9)) present the basis for the thermoelectric effects and their theoretical calculation.

\subsubsection{The Seebeck effect}

The Seebeck effect occurs when a temperature gradient is created across a material in an open-circuit condition, in which no electric current is flowing. Since $\mathbf{J}=0$ in this case, equation (1.3) yields (expressed in finite differences):

$$
V=\Delta \Phi=-\frac{L_{12}}{L_{11}} \Delta T=-S \Delta T \text {. }
$$


In this equation, the Seebeck coefficient $S$ is defined as:

$$
S=\frac{L_{12}}{L_{11}}=-\frac{1}{e T} \frac{\mathcal{L}_{1}}{\mathcal{L}_{0}}
$$

Here, the relations (1.5) and (1.6) were used to put the Seebeck coefficient in terms of the moments (1.8) and (1.9), respectively. Further, it follows from the definitions of the latter that $L_{11}$ equals the conductivity $\sigma$. With these results, the first kinetic equation for the electric current density can be written as:

$$
\mathbf{J}=\sigma \Delta \Phi+\sigma S \Delta T
$$

Phenomenologically, the Seebeck effect can be understood as follows: The electrons at the hot end of the material, on which the temperature gradient is applied, possess a higher thermal energy than the electrons at the cold end. As a consequence, the velocity of the electrons at the hot end is also higher, leading to a net diffusion current of the electrons from the hot to the cold end. At the latter, electrons accumulate, giving rise to an electric field in the material which counteracts the thermal diffusion current and leads to a steady-state voltage that can be measured in an open-circuit condition.

The experimental determination of the absolute Seebeck coefficient is, however, not trivial ${ }^{19}$. An experimental voltage measurement on a thermocouple is depicted in Fig. 1.1. Two metals, $A$ and $B$, are joined at two temperatures, $T_{1}$ and $T_{2}>T_{1}$. In an experiment, the voltmeter that is connected to one of the materials has in general a third temperature $T^{\prime}$. By applying eq. (1.10) to the three parts of the

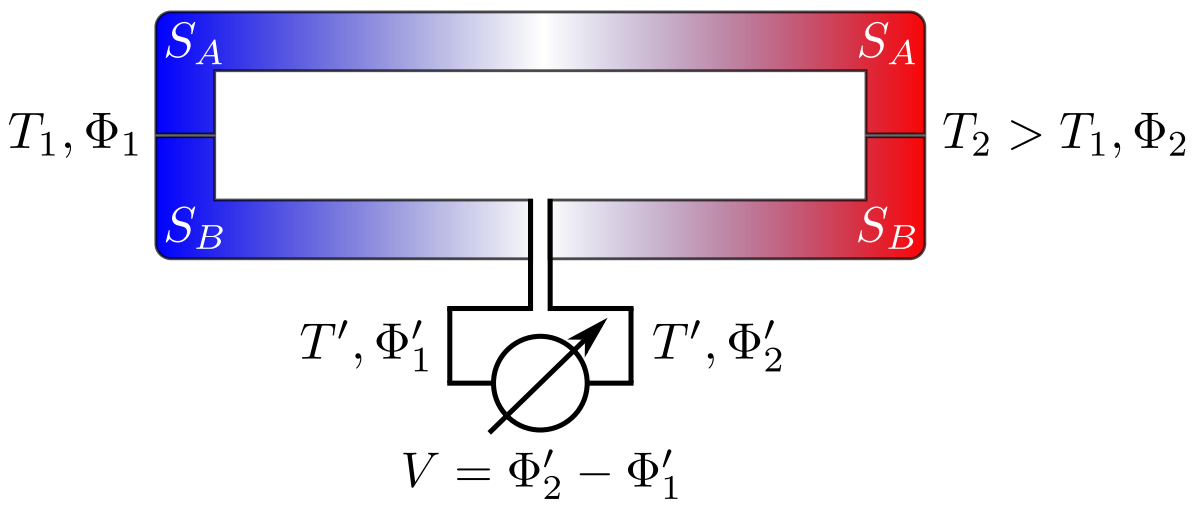

Figure 1.1: Measurement of the Seebeck effect. Two metals $A$ and $B$ are joined at the temperatures $T_{1}$ and $T_{2}$. A voltage can only be detected at the voltmeter when the two metals have different Seebeck coefficients, as indicated by eq. (1.13). 
thermocouple, the following relations are obtained:

$$
\begin{aligned}
& \Phi_{2}-\Phi_{2}^{\prime}=-S_{B}\left(T_{2}-T^{\prime}\right), \\
& \Phi_{2}-\Phi_{1}=-S_{A}\left(T_{2}-T_{1}\right), \\
& \Phi_{1}^{\prime}-\Phi_{1}=-S_{B}\left(T^{\prime}-T_{1}\right) .
\end{aligned}
$$

Hence, the voltage $V$ detected by the voltmeter yields:

$$
V=\Phi_{2}^{\prime}-\Phi_{1}^{\prime}=\left(S_{B}-S_{A}\right)\left(T_{2}-T_{1}\right)
$$

The obtained voltage in the experimental scenario is a measure for the difference in Seebeck coefficients of the two materials involved. It is obvious that no voltage would have been measured if only one material $\left(S_{B}=S_{A}\right)$ had been used for the experiment.

\subsubsection{The Peltier effect}

The Peltier effect occurs when an electron current is flowing through a material under isothermal conditions. First, the kinetic equations are modified to describe the Peltier effect. It is possible to express the heat current density $\mathbf{J}_{Q}$ (eq. (1.4)) in terms of the electric current density $\mathbf{J}$ (eq. (1.3)). By solving eq. (1.3) for $(-\nabla \Phi)$ and using $L_{21}=T L_{12}$ (eq. (1.6)), one obtains for the heat current density:

$$
\mathbf{J}_{Q}=T \frac{L_{12}}{L_{11}} \mathbf{J}_{e}+\frac{L_{11} L_{22}-L_{12} L_{21}}{L_{11}}(-\nabla T) .
$$

If no electron current is present, the coefficient connecting the heat current density caused by a temperature gradient is the thermal conductivity ${ }^{18} \kappa=-\frac{\mathbf{J}_{Q}}{\nabla T}=$ $\frac{L_{11} L_{22}-L_{12} L_{21}}{L_{11}}$. Further, the Seebeck coefficient $S=\frac{L_{12}}{L_{11}}$ appears in equation (1.14), so that the heat current density becomes:

$$
\mathbf{J}_{Q}=T S \mathbf{J}-\kappa \nabla T .
$$

Due to $\nabla T=0$ in the isothermal material considered for the Peltier effect, the relation between heat and electron current densities becomes:

$$
\mathbf{J}_{Q}=T S \mathbf{J}=\Pi \mathbf{J},
$$

in which the Peltier coefficient,

$$
\Pi=T S,
$$

describes the amount of heat carried by an electron current under isothermal conditions. 


\subsubsection{The Thomson effect}

If a temperature gradient is applied in addition to a current flow in a material that is connected to heat reservoirs at each end, the Thomson effect describes the change in heat flow that depends on the direction of the electric current. To derive the Thomson coefficient, one starts again with eq. (1.1), which gives in combination with $\mathrm{d} q=T \mathrm{~d} s$ and taking the time derivative ${ }^{19}$ :

$$
\frac{\mathrm{d} q}{\mathrm{~d} t}=\frac{\mathrm{d} u}{\mathrm{~d} t}-\mu \frac{\mathrm{d} n}{\mathrm{~d} t}
$$

By using the continuity equation for the particle current $\mathbf{J}_{N}$ and by describing the change of total energy $u$ by means of heat and electric current, taking into account the build-up of an electric field, it is possible to show that the rate of heat change is given by ${ }^{19}$ :

$$
\frac{\mathrm{d} q}{\mathrm{~d} t}=-\nabla \cdot \mathbf{J}_{Q}-\nabla \Phi \cdot \mathbf{J} .
$$

Inserting eq. (1.15) into eq. (1.18), the assumption of a uniform electric current $(\nabla \cdot \mathbf{J}=0)$ leads to

$$
\frac{\mathrm{d} q}{\mathrm{~d} t}=-\mathbf{J} \cdot \nabla(T S)+\nabla(\kappa \nabla T)-\nabla \Phi \cdot \mathbf{J}
$$

Since $S=S(T)$, the gradient can be written as $\nabla S=\frac{\mathrm{d} S}{\mathrm{~d} T} \nabla T$. Additional insertion of eq. (1.12) solved for $\nabla \Phi$ gives

$$
\frac{\mathrm{d} q}{\mathrm{~d} t}=-T \frac{\mathrm{d} S}{\mathrm{~d} T} \mathbf{J} \cdot \nabla T+\nabla(\kappa \nabla T)+\frac{1}{\sigma} \mathbf{J}^{2} .
$$

The last term in this equation $\left(\frac{1}{\sigma} \mathbf{J}^{2}\right)$ is the Joule heat that occurs even without an existing temperature gradient in every conductor due to its resistivity and is independent of current direction. The second term is the change in heat as given by the heat conduction equation that follows from the Fourier law. This term only contributes when the temperature gradient is not constant throughout the material. The first term is the Thomson heat which depends on the temperature gradient and the direction of the electric current. Consequently, the Thomson coefficient $\beta$ is defined as

$$
\beta=T \frac{\mathrm{d} S}{\mathrm{~d} T} .
$$

\subsubsection{Thermomagnetic effects}

In metals, thermomagnetic effects arise when an external magnetic field $H_{\text {ext }}$ is applied perpendicular to the plane in which heat and electric currents flow ${ }^{18}$ (Fig. 1.2a). In the following, the convention is used that electric currents, temperature gradients 
or voltages are applied in $x$-direction, i.e. between $x_{1}$ and $x_{2}$ in Fig. 1.2. Further, the following effects - if applicable - are called isothermal if the temperature gradient in $y$-direction is set to zero, or adiabatic if the heat current in $y$-direction is set to zero ${ }^{18}$. The complete set of effects is addressed in detail in ref. [18], whereas only a few examples are discussed here.

In the situation in which no temperature gradient is applied and an electric current is driven in $x$-direction, the Hall effect leads to the build-up of a voltage in $y$-direction, i.e. between $y_{1}$ and $y_{2}$ in Fig. 1.2a. If, on the other hand, no electric current is driven through the material, but a temperature gradient is applied between $x_{1}$ and $x_{2}$, a voltage in $y$-direction can be detected again. This is the so-called Nernst effect. The Ettingshausen effect describes the build-up of a temperature gradient in $y$-direction that is generated by an electric current driven in $x$-direction. Finally, if the temperature gradient between $y_{1}$ and $y_{2}$ is caused by an applied temperature gradient along the $x$-direction, this effect is called the Leduc-Righi effect.

To describe the effects above, kinetic equations can be constructed ${ }^{18}$ as in eqs. (1.3) and (1.4). The equations are developed for electric and heat currents in $x$ - and $y$-direction. The gradients of the temperature and the electrochemical potential are also split into the two cartesian coordinates. Thus, one ends up with four equations containing four kinetic coefficients each. The Onsager relations in the presence of an external magnetic field state that ${ }^{18}$

$$
L_{i j}\left(H_{\text {ext }}\right)=L_{j i}\left(-H_{\text {ext }}\right) .
$$

The use of this relation, combined with the assumption that the material is isotropic in the plane, leads to a reduction of the kinetic coefficients to $\operatorname{six}^{18}$. Furthermore,

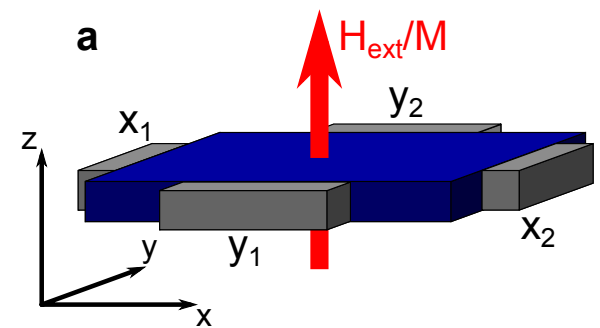

b

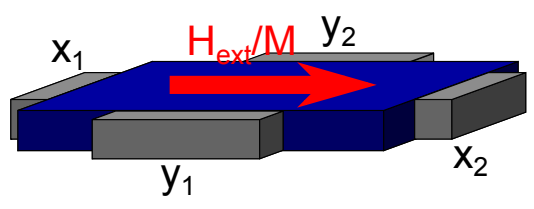

Figure 1.2: Geometries for thermomagnetic effects. a, Thermomagnetic effects occur when an external magnetic field is applied perpendicular to the $x$-y-plane, in which heat and electric currents flow. Spin caloritronic effects occur in this geometry in the absence of an external magnetic field due to spin-orbit interaction, when spin currents in normal metals are regarded. In ferromagnetic materials, the magnetization aligned as indicated, the geometry gives rise to "anomalous" thermomagnetic effects. b. In ferromagnetic materials, this geometry leads to "planar" thermomagnetic effects. 
assigning coefficients for the above mentioned effects, the dynamical equations can be written in matrix form

$$
\left(\begin{array}{c}
\nabla_{x} \Phi \\
\nabla_{y} \Phi \\
J_{x}^{Q} \\
J_{y}^{Q}
\end{array}\right)=\left(\begin{array}{cccc}
\sigma_{i}^{-1} & H_{\mathrm{ext}} R_{i} & -S & -H_{\mathrm{ext}} \eta_{i} \\
-H_{\mathrm{ext}} R_{i} & \sigma_{i}^{-1} & H_{\mathrm{ext}} \eta_{i} & -S \\
-T S & -T H_{\mathrm{ext}} \eta_{i} & -\kappa_{i} & -H_{\mathrm{ext}} \kappa_{i} \mathfrak{L} \\
T H_{\mathrm{ext}} \eta_{i} & -T S & H_{\mathrm{ext}} \kappa_{i} \mathfrak{L} & -\kappa_{i}
\end{array}\right)\left(\begin{array}{c}
J_{x} \\
J_{y} \\
\nabla_{x} T \\
\nabla_{y} T
\end{array}\right),
$$

in which the index $i$ denotes isothermal coefficients, $R$ the Hall coefficient, $\eta$ the Nernst coefficient and $\mathfrak{L}$ the Leduc-Righi coefficient.

If spin currents are considered in a normal metal in the absence of an external magnetic field, the spin-orbit interaction can lead to new spin caloritronic effects that are labeled according to their properties and the above definitions, but with the prefix "spin".

In ferromagnetic metals, the thermomagnetic effects can be classified depending on the direction of the magnetization. If the magnetization is perpendicular to the plane, as in Fig. 1.2a, the effects are labeled with "anomalous" as prefix, e.g. anomalous Nernst effect. If the magnetization is in the plane, as in Fig. 1.2b, the effects are labeled with the prefix "planar", e.g. planar Nernst effect" ${ }^{11}$

\subsubsection{Spin-dependent effects in metallic magnetic multilayers}

In metallic multilayers that contain ferromagnetic metals, such as GMR and tunnel magnetoresistance (TMR) layer stacks, the spin transport can conveniently be described with an independent-electron approach using a two current model. For each of the two spin types, a conductance channel is used to describe the transport of the respective spins in the multilayer ${ }^{11}$. The transport in GMR and TMR devices that contain two metallic, ferromagnetic layers with collinearly, i.e. parallel or antiparallel, aligned magnetizations is of particular interest. In the following, the kinetic equations for GMR stacks are introduced, whereas the transport in tunnel junctions is treated later in this chapter.

In the two current model, considering only linear response, the charge current $\mathbf{J}=\mathbf{J}_{\uparrow}+\mathbf{J}_{\downarrow}$, spin current $\mathbf{J}_{s}=\mathbf{J}_{\uparrow}-\mathbf{J}_{\downarrow}$ and heat current $\mathbf{J}^{Q}=\mathbf{J}_{\uparrow}^{Q}+\mathbf{J}_{\downarrow}^{Q}$ can be cast into matrix form as a function of the driving forces electrochemical potential $\mu_{c}=\frac{\mu_{\uparrow}+\mu_{\downarrow}}{2}$, spin accumulation $\mu_{s}=\mu_{\uparrow}-\mu_{\downarrow}$ and temperature $T$ (ref. [11]):

$$
\left(\begin{array}{c}
\mathbf{J} \\
\mathbf{J}_{s} \\
\mathbf{J}^{Q}
\end{array}\right)=\sigma\left(E_{F}\right)\left(\begin{array}{ccc}
1 & P & S T \\
P & 1 & P^{\prime} S T \\
S T & P^{\prime} S T & \frac{\kappa T}{\sigma}
\end{array}\right)\left(\begin{array}{c}
\frac{1}{e} \nabla \mu_{c} \\
\frac{1}{2 e} \nabla \mu_{s} \\
-\frac{1}{T} \nabla T
\end{array}\right) .
$$

Here, $\sigma\left(E_{F}\right)=\sigma_{\uparrow}\left(E_{F}\right)+\sigma_{\downarrow}\left(E_{F}\right)$ is the total conductivity at the Fermi level, $P=$ $\left.\frac{\sigma_{\uparrow}-\sigma_{\downarrow}}{\sigma}\right|_{E_{F}}$ is the spin polarization of the conductivity, $P^{\prime}=\left.\frac{\partial(P \sigma)}{\partial E}\right|_{E_{F}}$ its energy 
derivative, $S=\frac{\sigma_{\uparrow} S_{\uparrow}+\sigma_{\downarrow} S_{\downarrow}}{\sigma}$ is the total Seebeck coefficient and $\kappa=\kappa_{\uparrow}+\kappa_{\downarrow}$ the total thermal conductivity. The $P^{\prime} S T$ entries in the matrix equation (1.22) give rise to the spin-dependent Seebeck and the spin-dependent Peltier effect. For both effects, spin-dependent coefficients can be defined as follows ${ }^{23,24}$ :

$$
S_{s}=S_{\uparrow}-S_{\downarrow}, \quad \Pi_{s}=T S_{s}=\Pi_{\uparrow}-\Pi_{\downarrow} .
$$

Recently, these two effects were observed experimentally ${ }^{23,24}$ (see section 1.3).

\subsection{The spin Seebeck effect}

In 2008, Uchida et al. ${ }^{10}$ discovered an unexpected new effect that they called spin Seebeck effect. The authors of this report prepared $20 \mathrm{~nm}$-thick permalloy films that were patterned into strips with lengths and widths in the order of millimeters. At the ends, $10 \mathrm{~nm}$-thick platinum strips were patterned across the permalloy strips (Fig. 1.3e). Uchida et al. observed a spin current being injected into the platinum strips if the permalloy was magnetized in the plane and a temperature gradient applied collinearly, as indicated in Fig. 1.3e. The spin current in the Pt strips is detected via a voltage generated by the inverse spin Hall effect, which originates from the spin-orbit interaction in the paramagnetic metal (Fig. 1.3c). In this transverse configuration, the spin Seebeck effect was also reported for ferromagnetic insulators (ref. [25], Fig. 1.3g) and ferromagnetic semiconductors ${ }^{26}$. In 2010, Uchida et al. ${ }^{27}$ reported that the spin Seebeck effect can also be observed in the longitudinal configuration that is sketched in Fig. 1.3d. For the experiments, the ferromagnetic insulator yttrium-iron-garnet (YIG) was used (Fig. 1.3f, ref. [27]). The existence of the effect in insulators and the length scales on which the effect occurs suggest that the spin Seebeck effect is mediated by magnons (Fig. 1.3b) and phonon-magnon interaction, and that spin transport due to conduction electrons is negligible ${ }^{11}$. Therefore, according to Bauer et al. ${ }^{11}$, the effect should be labeled magnonic Seebeck effect, but the name spin Seebeck effect is still widely used in literature.

At the time of this writing, a vivid discussion on the contamination of the spin Seebeck signal by other effects persists in the magnetism community. Especially when thick substrates are used, the temperature gradient can deviate from perfect in-plane alignment and the measured transverse spin Seebeck signals could be influenced by longitudinal spin Seebeck and anomalous Nernst signals (ref. [28] and refs. therein). In addition, Avery et al. tested the transverse spin Seebeck effect in permalloy films grown on $500 \mathrm{~nm}$-thin $\mathrm{Si}-\mathrm{N}$ membranes, in which no deviation of the in-plane temperature gradient caused by substrate influence is expected ${ }^{28}$. In these experiments, however, only signatures of the planar Nernst effect were observed.

These issues do not apply to the longitudinal configuration (Fig. 1.3d), where the temperature gradient is applied perpendicularly to the sample plane, collinearly to 
the injected spin current. Using this configuration, Chien's group reported on induced magnetic moments in Pt at the Pt/YIG interface generated by a proximity effect $^{29-31}$. These magnetic moments could also distort the detected spin Seebeck voltage. On the other hand, Geprägs et al. could not find any evidence for the proximity effect in $\mathrm{Pt} / \mathrm{YIG}$ bilayers $^{32}$ in their samples. In element-specific X-ray magnetic dichroism measurements no magnetic signal was found for $7 \mathrm{~nm}$ and $10 \mathrm{~nm}$ Pt films, and only a negligible magnetic moment of $0.003 \mu_{B}$ for $3 \mathrm{~nm}$ Pt.

In the longitudinal configuration, the spin Seebeck effect (LSSE) coincides with the anomalous Nernst effect (ANE) and a careful disentanglement of the two effects is necessary. Meier et al. performed longitudinal spin Seebeck measurements on $\mathrm{NiFe}_{2} \mathrm{O}_{4} / \mathrm{Pt}$ bilayers with the aim of distinguishing between the ANE and the $\mathrm{LSSE}^{33}$. Since the $\mathrm{NiFe}_{2} \mathrm{O}_{4}$ is a semiconducting ferrimagnet, the contribution of the ANE to the detected signal can be controlled by the base temperature of the film,

a Conduction-election spin current

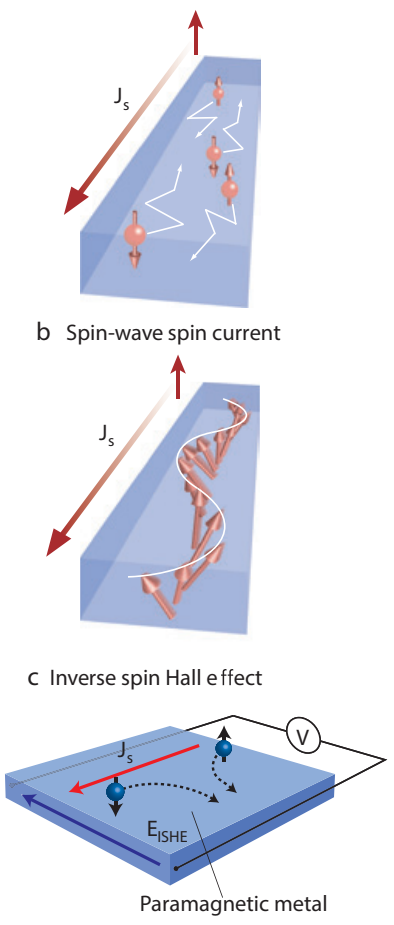

d Longitudinal configuation e Transverse configuration

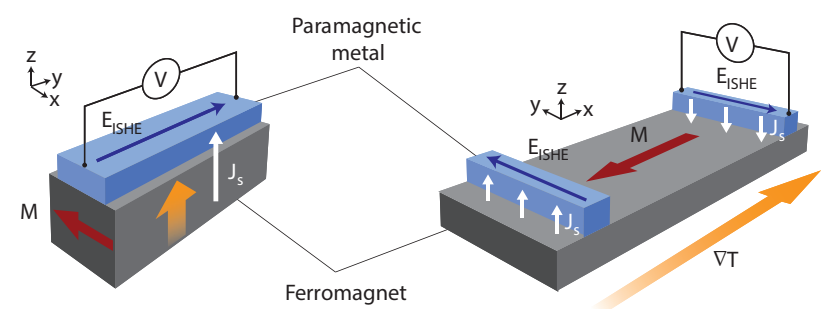

$\mathrm{f}$

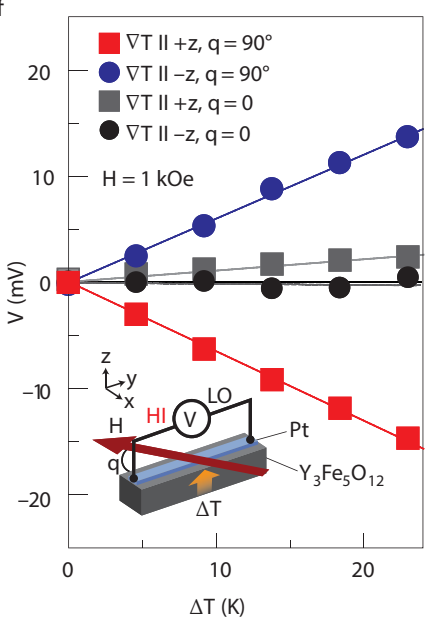

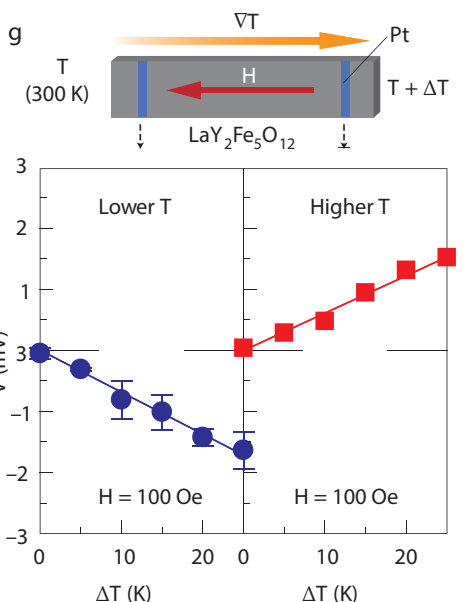

Figure 1.3: The spin Seebeck effect. a, Spin current mediated by conduction electrons. b, Spin current due to a magnon. c, Principle of the inverse spin Hall effect. d, f, Configuration and experimental data for the longitudinal spin Seebeck effect. e, $\mathbf{g}$, Configuration and experimental data for the transverse spin Seebeck effect. Taken from ref. [11]. () 2012 NPG. 
because the ANE requires free charge carriers. The authors were able to disentangle the superposition of ANE and LSSE signals, since an increase of ANE contribution with increasing temperature is found that is similar to the temperature dependence of the conductivity ${ }^{33}$.

\subsection{Spin-dependent Seebeck and Peltier effects in spin valves}

The spin-dependent thermoelectric effects that were introduced at the end of section 1.1 were studied first by Shi et al. on Co/Cu GMR multilayers in $1993^{6}$. Further experiments on thermally driven spin transport through GMR multilayers were carried out a decade ago by Gravier et al. on $\mathrm{Co} / \mathrm{Cu}$ nanowires ${ }^{7,8}$. In these experiments, evidence for a spin-dependent Peltier effect was already found ${ }^{9}$. Recently, the research group of van Wees studied the spin-dependent thermoelectric effects on non-local GMR spin valves in detail ${ }^{23,24,34}$.

Slachter et $a l .{ }^{23}$ were able to show that a spin current can be thermally injected into a normal metal by using a non-local spin valve, as depicted in Fig. 1.4. In this experiment, a first ferromagnetic layer $\left(\mathrm{FM}_{1}\right.$ in Fig. 1.4) is heated by an electric current (Joule heating). This heating generates a temperature gradient in the adjacent normal metal. To study the spin dependence of the Seebeck coefficient in the first ferromagnet, a second ferromagnetic layer $\left(\mathrm{FM}_{2}\right.$ in Fig. 1.4) is placed as a detector within the spin diffusion length of the normal metal. If different Seebeck

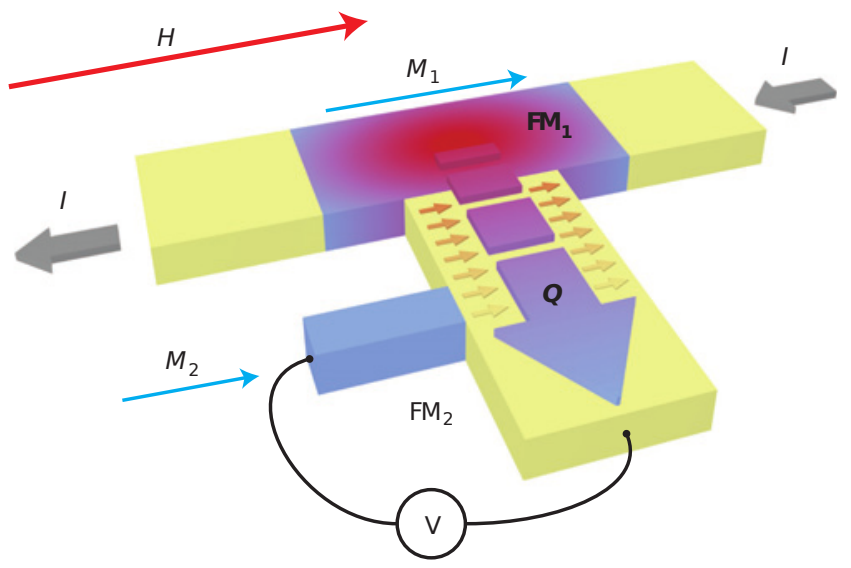

Figure 1.4: Device for detection of the spin-dependent Seebeck effect. The non-local spin valve consists of two ferromagnetic layers $\left(\mathrm{FM}_{1}, \mathrm{FM}_{2}\right.$, made of permalloy) that are connected via a copper layer. The Joule heating caused by an electrical current flowing through $\mathrm{FM}_{1}$ generates a heat current into the adjacent normal metal. The second ferromagnet $\mathrm{FM}_{2}$ acts as a detector for a spin accumulation close to the interface. Taken from ref. [23]. ㅇ 2010 NPG. 
coefficients exist for the two spin channels in the ferromagnet, a difference in voltage should be measured for the two magnetizations $\mathrm{M}_{1}$ and $\mathrm{M}_{2}$, aligned parallel or antiparallel, respectively. The signal of the thermally driven spin injection is expected to scale with the Joule heating, that means with the square of the current driven through $\mathrm{FM}_{1}{ }^{23}$. In a device as shown in Fig. 1.4, in which permalloy $\left(\mathrm{Ni}_{80} \mathrm{Fe}_{20}\right)$ is used as ferromagnetic material and copper as normal metal, a spin-dependent Seebeck coefficient of $S_{s}=-3.8 \mu \mathrm{V} \mathrm{K}^{-1}$ is observed. The authors also investigate electrical spin injection in these devices and compare electrical and thermal spin injection efficiency. Based on their results, Slachter et al. claim that thermal spin injection might be a feasible alternative to electrical spin injection ${ }^{23}$.

Furthermore, the group of van Wees was also the first to directly observe the spin-dependent Peltier effect in a single GMR stack ${ }^{24}$. Flipse et al. constructed a GMR spin valve in perpendicular geometry, as shown in Fig. 1.5. Several bottom and top leads to the device can be used for four probe electrical measurements. In addition, a platinum-constantan-thermocouple is brought close to the GMR stack to measure temperature differences generated by the (spin-dependent) Peltier effect. The measurement procedure is depicted in Fig. 1.5b: An electrical current is sent through the GMR stack using the contacts 1 and 2. Concurrently, the Seebeck voltage at the thermocouple is recorded. Due to the large Seebeck coefficient of

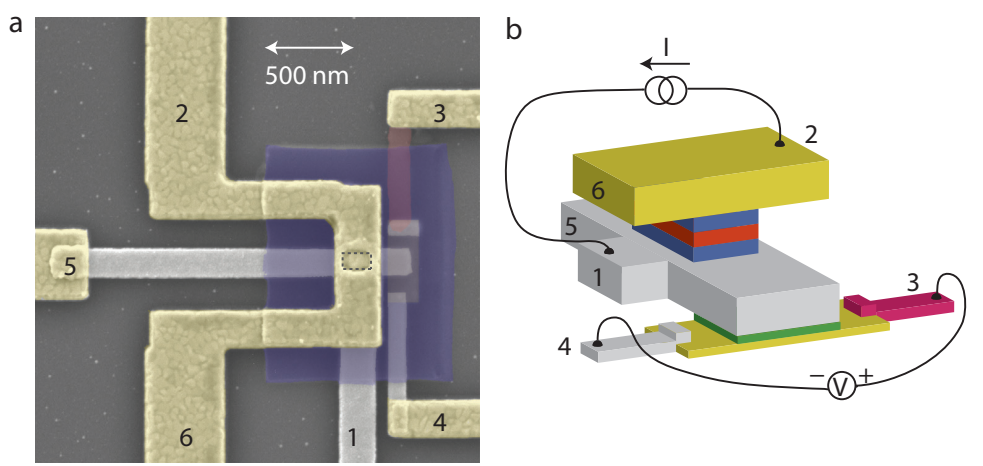

Figure 1.5: Direct measurement of the spin-dependent Peltier effect. a, Scanning electron microscope image of the device used for detection of the spindependent Peltier effect. For the yellow colored leads, gold is used. The blue area consists of cross-linked PMMA to insulate the buried GMR device. As bottom contact, platinum is used (grey). The constantan $\left(\mathrm{Ni}_{45} \mathrm{Cu}_{55}\right)$ part of the thermocouple is depicted in red. $\mathbf{b}$, Sketch of the device and the measurement procedure. A current is driven through the GMR stack consisting of permalloy and copper (contacts 1 and 2). The temperature difference generated by the spin-dependent Peltier effect is detected using an electrically insulated thermocouple (the insulating $\mathrm{Al}_{2} \mathrm{O}_{3}$ layer is sketched in green). Taken from ref. [24]. ㅇ 2012 NPG. 
constantan $\left(-32 \mu \mathrm{VK}^{-1}\right)$, even small temperature differences in the bottom lead can be detected ${ }^{24}$. The measured temperature difference is related to the conductivity spin polarization $P_{\sigma}=\frac{\sigma_{\uparrow}-\sigma_{\downarrow}}{\sigma_{\uparrow}+\sigma_{\downarrow}}$ and to the spin-dependent Peltier coefficient $\Pi_{s}=\Pi_{\uparrow}-\Pi_{\downarrow}$ via

$$
\Delta T=\frac{\sigma}{4 \kappa}\left(1-P_{\sigma}^{2}\right) \Pi_{s} \mu_{s}^{0}
$$

in which $\sigma$ is the total electrical conductivity, $\kappa$ the thermal conductivity and $\mu_{s}^{0}$ the spin accumulation ${ }^{24}$. The authors observed temperature differences of $\Delta T=$ $3 \mathrm{mK}$, in agreement with finite-element simulations of the heat and spin transport, combined with eq. (1.24). Flipse et al. further determined $P_{\sigma}$ in additional electrical four probe measurements. Combining the experimental results with the finite-element modelling, they obtained a spin-dependent Peltier coefficient $\Pi_{s} \approx-0.9 \mathrm{mV}$ for permalloy. Using the relation between Seebeck and Peltier coefficients (eq. (1.17)), the authors obtained a Seebeck coefficient of $-3.0 \mu \mathrm{V} \mathrm{K}{ }^{-1}$, in accordance with their earlier results obtained in non-local spin valves ${ }^{24}$.

\subsection{Spin-dependent Seebeck effects in tunneling systems}

Several experiments on thermoelectric effects in magnetic tunneling systems have been carried out by other research groups concurrently with the work presented in the next chapters. Those works include thermal spin injection into a semiconductor, Seebeck effects in tunnel junctions with alumina $\left(\mathrm{Al}_{2} \mathrm{O}_{3}\right)$ barriers and in the Co$\mathrm{Fe}-\mathrm{B} / \mathrm{MgO}$ system, tested by resistive heating in the latter case. Furthermore, a short introduction to the theoretical predictions of the thermal spin-transfer torque is given.

\subsubsection{Seebeck spin tunneling into silicon}

Efficient spin injection into semiconductors is an important issue with respect to the integration of spintronic devices with existing logic devices based on semiconductor technologies. For this reason, Le Breton et al. studied the possibility of thermal spin injection into silicon ${ }^{35}$. To investigate this effect, which is called Seebeck spin tunneling, ferromagnet/insulator tunnel contacts were placed onto silicon layers as sketched in the upper part of Fig. 1.6. The authors use permalloy $\left(\mathrm{Ni}_{80} \mathrm{Fe}_{20}\right)$ as a ferromagnet in combination with an $\mathrm{Al}_{2} \mathrm{O}_{3}$ tunnel barrier. A temperature difference between the ferromagnet and the silicon is created by Joule heating of the silicon layer ${ }^{35}$. The spin accumulation in the silicon close to the tunnel barrier is studied in the Hanle geometry: Given an in-plane magnetization of the ferromagnet and, thus, an in-plane orientation of the injected spins, an external magnetic field perpendicular to the plane leads to spin precession, which diminishes the spin accumulation in the silicon and results in a Lorentzian-shaped field dependence ${ }^{35}$. The lower left graph in Fig. 1.6 shows the voltage that drops across the tunnel 
junction, in addition to voltages caused by the resistances in the leads and the silicon when the silicon is heated. The same shape is obtained when the heating current direction in the silicon is reversed (open/closed circles in lower left graph in Fig. 1.6). This fact, the linear scaling of the amplitude (lower right graph in Fig. 1.6) and other test experiments lead to the conclusion that the generated spin accumulation in the silicon layer originates solely from thermal spin injection from the ferromagnet through the tunnel barrier ${ }^{35}$.

Le Breton et al. also performed electrical spin injection experiments to deduce the sign of the thermally generated spin accumulation from the electrically obtained data by comparison ${ }^{35}$ : When the silicon is heated with respect to the ferromagnetic layer, a positive spin accumulation is found, i.e. the magnetic moment of the spins in the silicon layer is parallel to the magnetization of the ferromagnet, whereas

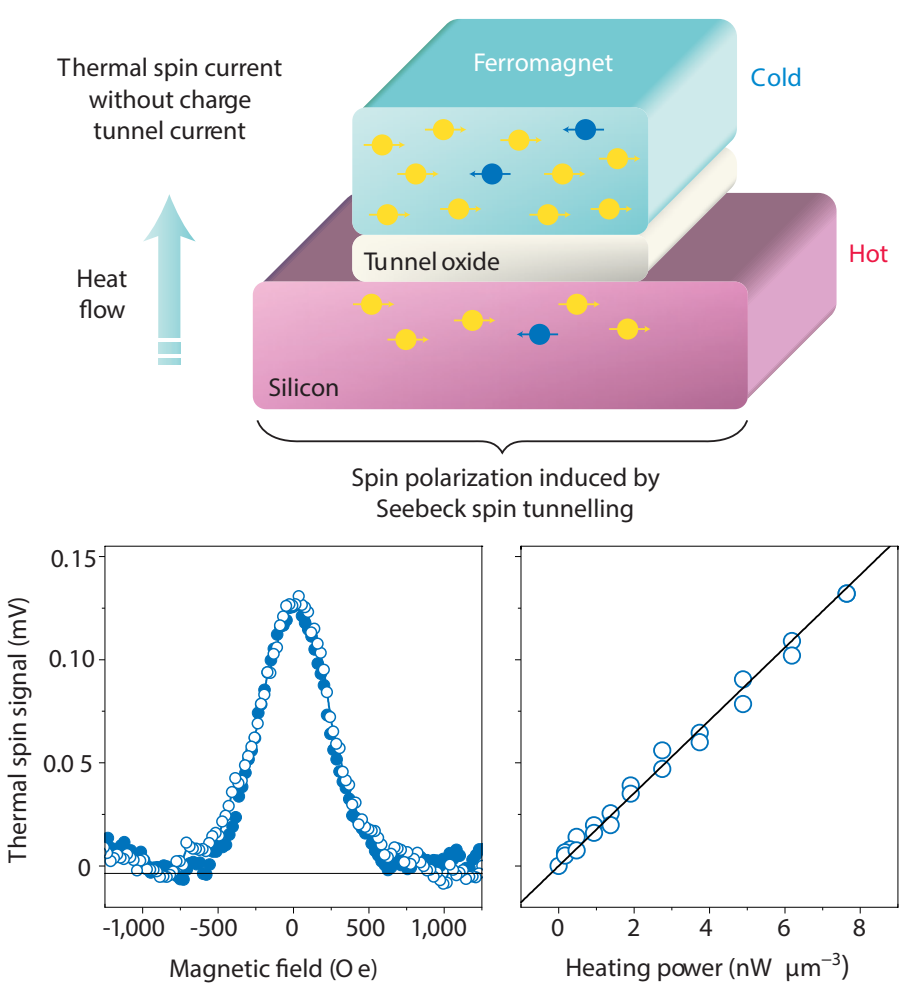

Figure 1.6: Seebeck spin tunneling into silicon. Upper part: A tunnel junction consisting of a ferromagnet and an oxide layer is placed onto a silicon layer. Joule heating is used to heat the silicon layer and to create a temperature difference across the tunnel barrier. The Hanle curves, shown in the lower left graph, and the linear dependence of the effect amplitude on the heating power, shown in the lower right graph, indicate the thermal spin injection into silicon. Taken from ref. [14]. (c) 2012 NPG. 
an inversion of the temperature gradient leads also to an inversion of the spin accumulation.

To predict the influences of certain parameters, such as the tunneling spin polarization, on the Seebeck spin tunneling, Le Breton et al. developed a free-electron model considering an elastic tunneling process. This model reveals amongst other things a strong dependence of the spin accumulation (in magnitude and sign) on the asymmetry of the tunnel spin polarization around the Fermi level. This fact also holds true for ferromagnet-insulator-ferromagnet tunnel junctions, as pointed out in the simultaneously published work that is presented in chapter 2 .

\subsubsection{The tunnel magneto-Seebeck effect}

In contrast to the experiments presented so far, it is more convenient to examine the thermoelectric coefficients in a ferromagnet-insulator-ferromagnet junction for the two collinear magnetization alignments parallel (P) and antiparallel (AP), instead of the spin-dependent definitions given in eq. (1.23). When $\theta$ is defined as the angle between the magnetization directions of the two ferromagnetic layers, the tunnel magneto-Seebeck ratio (TMS ratio) is given by ${ }^{36}$ :

$$
\mathrm{TMS}=\frac{S\left(0^{\circ}\right)-S(\theta)}{\min \left(\left|S\left(0^{\circ}\right)\right|,|S(\theta)|\right)},
$$

in which $S$ denotes the Seebeck coefficient, $\theta=0^{\circ}$ for $\mathrm{P}$ alignment and $\theta=180^{\circ}$ for AP alignment.

\section{The tunnel magneto-Seebeck effect in alumina tunnel junctions}

The tunnel magneto-Seebeck effect in tunnel junctions with alumina barriers was investigated by Lin et al. MTJs consisting of ferromagnetic $\mathrm{Co}_{90} \mathrm{Fe}_{10}$ layers separated by an amorphous $\mathrm{Al}_{2} \mathrm{O}_{3}$ tunnel barrier were studied using laser heating. In detail, the layer stack used in the experiments consists of ${ }^{37}: 5 \mathrm{~nm}$ Ta $/ 25 \mathrm{~nm} \mathrm{PtMn}$

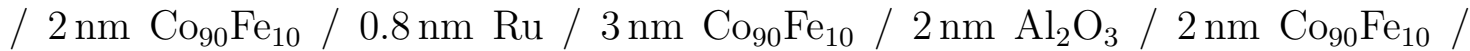
$5 \mathrm{~nm} \mathrm{Ni} \mathrm{Ni}_{80} \mathrm{Fe}_{20} / 4.8 \mathrm{~nm} \mathrm{Ru} / 10 \mathrm{~nm} \mathrm{Au}$. MTJs of $80 \mu \mathrm{m}$ diameter were patterned out of this layer stack, insulated with alumina, and $\mathrm{Ta} / \mathrm{Cu}$ layers were used as top contact $^{37}$.

A tunnel magnetoresistance (TMR) curve is shown in Fig. 1.7a. A TMR ratio of $40 \%$ is observed. Fig. 1.7b depicts measurements of the Seebeck voltage generated in the MTJ, when either the top or bottom contact is heated or no heat is applied. When the MTJ is heated by the laser from the top, a negative voltage is observed, whereas the sign of the Seebeck voltage changes when the temperature difference is inverted. Based on resistance changes in the top contacts during laser heating, Lin et al. estimate a temperature gradient of $1 \mathrm{~K}$ or less across the tunnel junction ${ }^{37}$. 

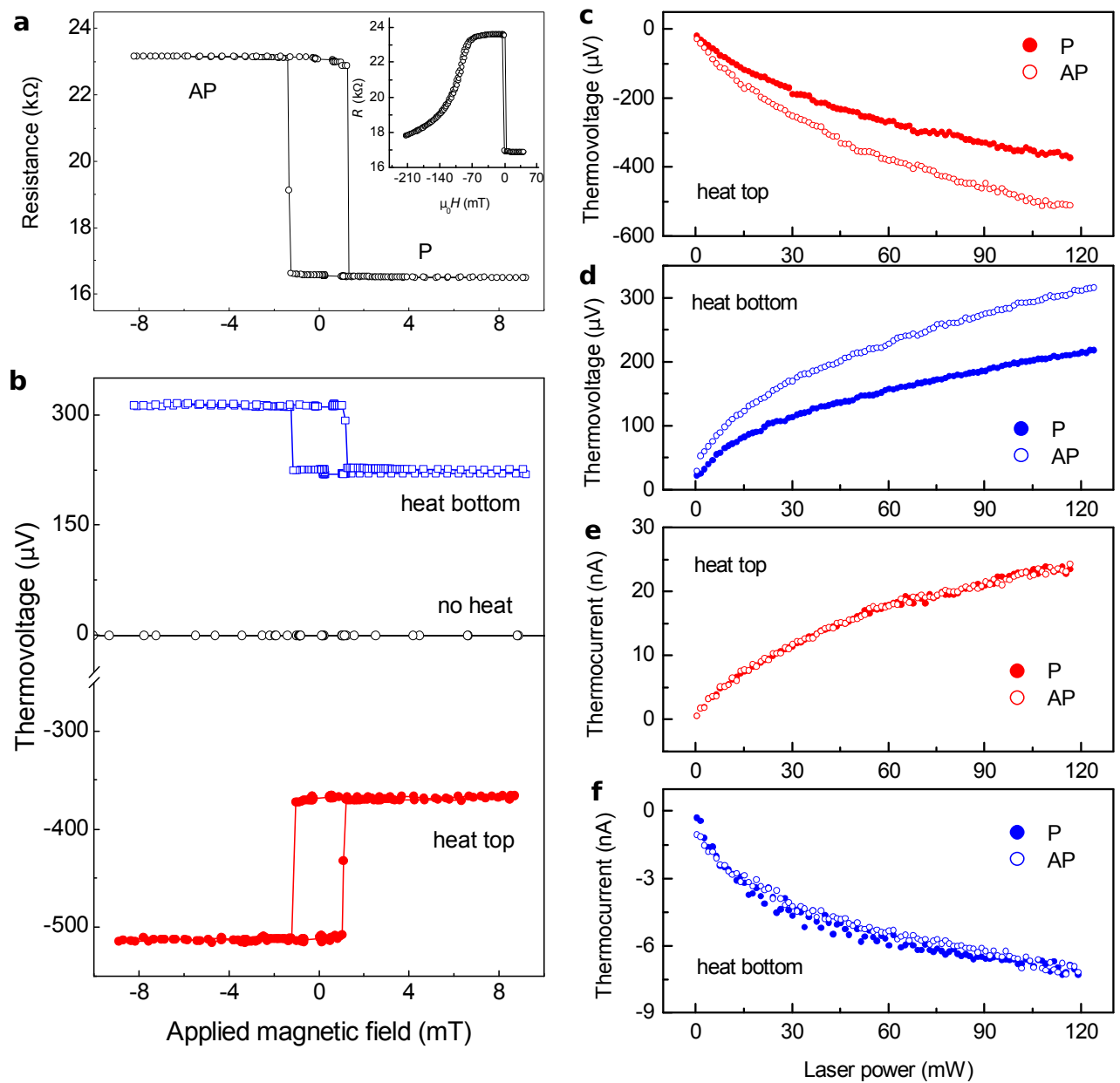

Figure 1.7: Tunnel magneto-Seebeck effect in alumina junctions. a, TMR curve. b, Seebeck voltage measurements in different heating configurations. c, d, Seebeck voltages as function of applied laser power. c, d, Thermocurrents as function of applied laser power. Taken from ref. [37]. () 2012 NPG.

Given the negative Seebeck voltages in this case, the authors calculate a positive Seebeck coefficient of $1000 \mu \mathrm{V} \mathrm{K}^{-1}$ according to eq. (1.11) as a lower estimate ${ }^{37}$.

In addition, the authors performed Seebeck voltage and thermocurrent measurements as a function of applied laser power (Fig. 1.7c-f). The tunnel magnetoSeebeck effect can be seen in Fig. 1.7c and Fig. 1.7d and amounts to a ratio of $40 \%$, which equals the TMR ratio ${ }^{37}$. As can be seen in Figs. 1.7e-f, Lin et al. do not observe any magnetic effect in the thermocurrent measurements.

The positive sign of the Seebeck coefficient and the absence of a magnetic effect in thermocurrent measurements are in contrast to the observations on $\mathrm{Co}-\mathrm{Fe}-\mathrm{B} / \mathrm{MgO}$ MTJs that are presented in the next section and in chapters 2-3. Lin et al. claim 
that the differences are due to the coherent tunneling process ${ }^{38}$ in $\mathrm{MgO}$ tunnel junctions and develop a model to explain the differences ${ }^{37}$ : The Jullière model is employed to describe the conductivities in parallel and antiparallel magnetization alignments. Eqs. (1.3) to (1.8) are then used to obtain expressions for the Seebeck coefficients and thermocurrents, which support the experimental observations ${ }^{37}$.

\section{Magneto-Seebeck effect in $\mathrm{MgO}$ based tunnel junctions with resistive heating}

The tunnel magneto-Seebeck effect in $\mathrm{Co}-\mathrm{Fe}-\mathrm{B} / \mathrm{MgO}$ tunnel junctions was studied simultaneously to the work presented in the following chapters by Liebing et al. ${ }^{39-41}$. Whereas laser heating is used in the thesis at hand to create temperature differences across the MTJ layer stack, Liebing et al. heated the MTJ by Joule heating of an additional heater line on top of the tunnel junction (Fig. 1.8). The layer stack of the MTJ is depicted in Fig. 1.8a. The temperature rise in the heater line was extracted from its electrical resistance at different heating powers (Fig. 1.8c,d). Heating powers up to $124 \mathrm{~mW}$ were possible ${ }^{41}$. Similar to the method used in the following chapters, the experimental parameters were used in finite-element simulations of the

a)

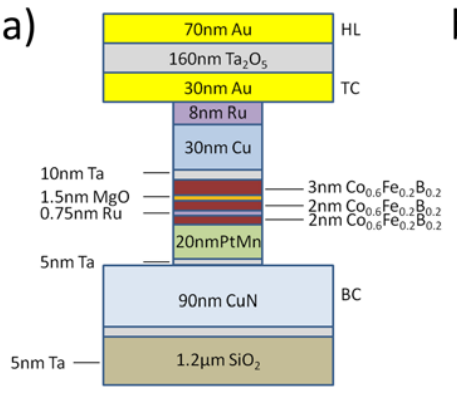

e)
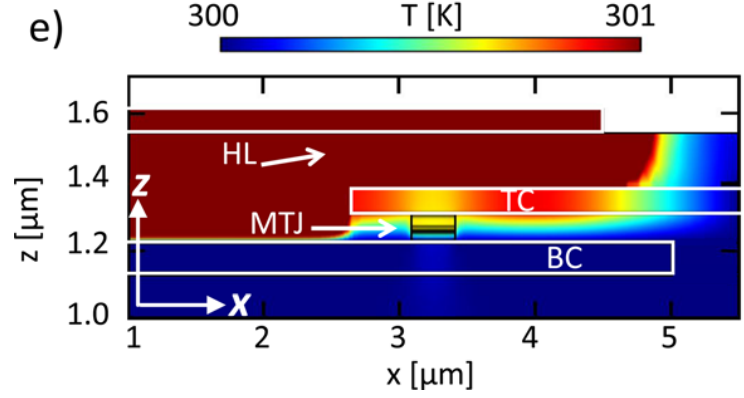

b)

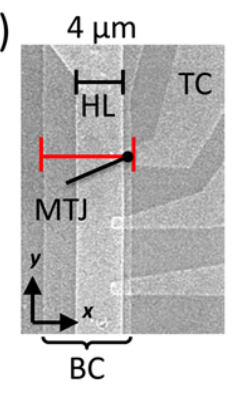

301

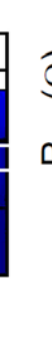

c)
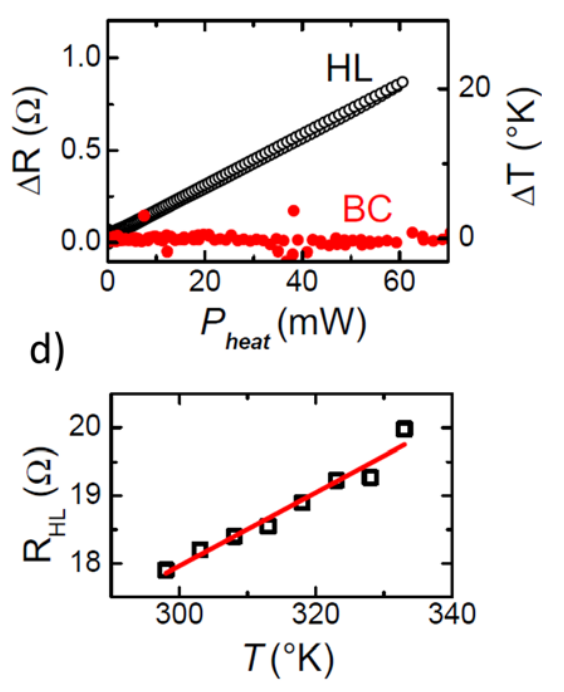

Figure 1.8: Resistive heating of a Co-Fe-B/MgO tunnel junction. a) MTJ layer stack. b) Scanning electron microscope image of the device. c) Resistance change and temperature rise for different heating powers. d) Temperature dependence of the heater line resistance. e) Finite-element simulations of the temperature distribution in the device. Taken from ref. [39]. () 2012 AIP Publishing LLC. 
heat transport through the MTJ (Fig. 1.8e) to estimate the temperature gradient across the tunnel barrier. For a heating power of $60 \mathrm{~mW}$, a temperature difference of $45 \mathrm{mK}$ was obtained ${ }^{39,41}$.

The left graph of Fig. 1.9 depicts the results of Seebeck voltage measurements on MTJs showing a TMR ratio between $70 \%$ and $140 \%$ that were obtained by Liebing et al. using different heating powers ${ }^{41}$. Seebeck voltages of up to $80 \mu \mathrm{V}$ and TMS ratios of $50 \%$ are observed. The TMS ratios decrease with increasing heating power, whereas the Seebeck voltages are proportional to the applied heating power ${ }^{41}$.

Liebing et al. also studied the thermocurrent in their $\mathrm{MTJs}^{41}$. The results are shown in the right graph of Fig. 1.9. Contrary to the observations of Lin et al. ${ }^{37}$, a magnetic switching of the thermocurrent is visible for all tested heating powers. The thermocurrents also scale linearly with the applied heating power, and thermocurrents calculated from the TMR and TMS measurements agree with the measured thermocurrents (dashed lines in the right graph of Fig. 1.9). The magnitudes of the Seebeck voltages, and the magnetic switching of the thermocurrents are in good agreement with the results obtained by laser heating which are presented in the remainder of this thesis.

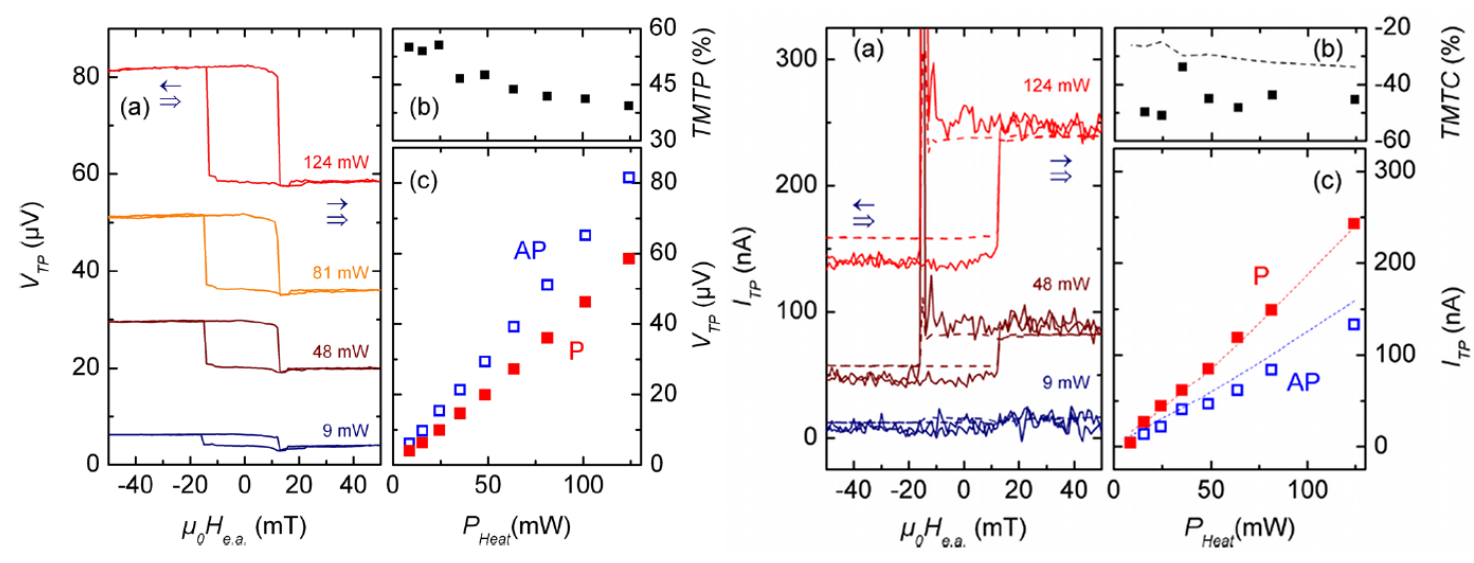

Figure 1.9: Resistive heating: Seebeck voltages and thermocurrents. Left graph: Seebeck voltages observed with different heating powers. Right graph: Thermocurrents measured at the same heating powers. Taken from ref. [41]. (c) 2013 AIP Publishing LLC.

\subsubsection{Thermal spin-transfer torque}

The existence of a thermal spin-transfer torque, that is the torque acting on a ferromagnetic layer's magnetization driven by a thermally induced spin-polarized current, was predicted by Hatami et al. for magnetoelectronic devices ${ }^{42}$, and by Slonczewski for a spin valve incorporating a ferromagnetic insulator as polarizing 


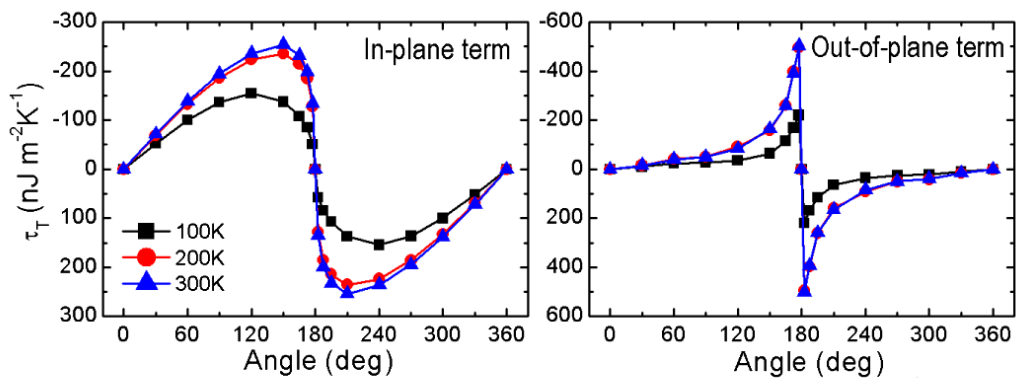

Figure 1.10: Angular dependence of thermal spin-transfer torque. In-plane and out-of-plane thermal spin transfer torques are shown for an $\mathrm{Fe} / \mathrm{MgO} / \mathrm{Fe} \mathrm{MTJ}$ with a 3 monolayer barrier at $T=300 \mathrm{~K}$. Taken from ref. [44]. (c) 2011 American Physical Society.

layer ${ }^{43}$. First $a b$ initio calculations of the thermal spin-transfer torque in epitaxial $\mathrm{Fe} / \mathrm{MgO} / \mathrm{Fe}$ MTJs were presented by Jia et $a l^{44}$. The results of that publication initiated the investigations of the parameter space for thermal spin-transfer torque in chapter 4 .

Jia et al. considered an epitaxial $\mathrm{Fe} / \mathrm{MgO} / \mathrm{Fe}$ magnetic tunnel junction to which a temperature gradient and/or a voltage can be applied. To calculate the thermal spin-transfer torque, the authors consider the spin current from one layer $n$ in the MTJ to the next $(n+1)$ (ref. [44]),

$$
\mathbf{J}_{n+1, n}=\frac{1}{8 \pi} \int \mathrm{d} E\left[\mathbf{t}_{n+1, n}^{L}(E) f_{L}(E)+\mathbf{t}_{n, n+1}^{R}(E) f_{R}(E)\right],
$$

in which $\mathbf{t}_{n+1, n}^{L}(E)$ and $\mathbf{t}_{n, n+1}^{R}(E)$ present the coefficients for spin transmission and $f_{L / R}(E)$ the Fermi-Dirac distribution functions of the left and right lead, respectively. When no external voltage is applied and only a temperature difference $\Delta T$ exists, the spin current can be expanded in terms of $\Delta T$. The difference of incoming and outgoing spin currents then gives the torque on a single layer and the total torque $\mathbf{T}_{\Delta T}$ can be obtained ${ }^{44}$ :

$$
\mathbf{T}_{\Delta T}=\frac{\Delta T}{e T_{0}} \int\left(E-E_{F}\right) \tau_{V}(E) \frac{\partial}{\partial E} f(E) \mathrm{d} E
$$

in which $\tau_{V}(E)$ is the electrical torkance. The thermal torkance $\tau_{T}=\mathbf{T}_{\Delta T} / \Delta T$ is shown in Fig. 1.10 for a 3 monolayer $\mathrm{MgO}$ barrier as a function of angle between the magnetizations of the two involved ferromagnetic layers. In a magnetic tunnel junction, both the in-plane and out-of-plane torques are important for the switching process $^{13}$. Both terms are in the order of $200 \mathrm{~nJ} \mathrm{~m}^{-2}$ at a temperature difference of $1 \mathrm{~K}$.

Jia et al. conclude from their results that thermal spin-transfer torque switching of MTJs is possible with temperature differences across the $\mathrm{MgO}$ barrier of $6.5 \mathrm{~K}$ 
for switching from antiparallel (AP) to parallel (P) magnetization alignment and $56.5 \mathrm{~K}$ to switch from $\mathrm{P}$ to $\mathrm{AP}^{44}$. Additionally, thermal torques are calculated for $\mathrm{MgO}$ barrier thicknesses of 5 and 7 monolayers. However, the results of Jia et al. predict that the thermal torques are already strongly reduced to $3 \mathrm{~nJ} \mathrm{~m} \mathrm{~m}^{-2} \mathrm{~K}^{-1}$ at 5 monolayers of $\mathrm{MgO}$. 



\title{
Chapter 2
}

\section{Seebeck effect in magnetic tunnel junctions}

\author{
M. Walter, J. Walowski, V. Zbarsky, et al. \\ Nature Materials 10, 742 (2011) \\ DOI: $10.1038 /$ nmat3076
}

\begin{abstract}
Creating temperature gradients in magnetic nanostructures has resulted in a new research direction, that is, the combination of magneto- and thermoelectric effects ${ }^{8,10,23,25,26}$. Here, we demonstrate the observation of one important effect of this class: the magnetoSeebeck effect. It is observed when a magnetic configuration changes the charge-based Seebeck coefficient. In particular, the Seebeck coefficient changes during the transition from a parallel to an antiparallel magnetic configuration in a tunnel junction. In this respect, it is the analogue to the tunnelling magnetoresistance. The Seebeck coefficients in parallel and antiparallel configurations are of the order of the voltages known from the charge-Seebeck effect. The size and sign of the effect can be controlled by the composition of the electrodes' atomic layers adjacent to the barrier and the temperature. The geometric centre of the electronic density of states relative to the Fermi level determines the size of the Seebeck effect. Experimentally, we realized 8.8\% magneto-Seebeck effect, which results from a voltage change of about $-8.7 \mu \mathrm{V} \mathrm{K}^{-1}$ from the antiparallel to the parallel direction close to the predicted value of $-12.1 \mu \mathrm{V} \mathrm{K}^{-1}$. In contrast to the spin-Seebeck effect, it can be measured as a voltage change directly without conversion of a spin current.
\end{abstract}

The creation of an electric field by a temperature gradient in a material has been known as the Seebeck effect since 1826. In recent years new spin-dependent thermal effects have been discovered in ferromagnets and the Seebeck effect is receiving renewed interest. The transport of heat and spin in magnetic nanostructures is described in ref. [8]. The spin-Seebeck effect driving this field was experimentally found, for example, in nanoscale metal structures ${ }^{23}$ and in magnetic insulators and semiconductors ${ }^{25,26}$. A strong asymmetry of the density of states with respect to the Fermi level promotes the heat-driven electron transport that leads to the 
common charge-Seebeck effect. These strong asymmetries can be found in the spinsplit density of states in ferromagnetic materials. Previously, the effect amplitude resulting from this spin asymmetry was believed to be a second-order effect. In this work, we demonstrate that the magneto-Seebeck effect can be large. We first present $a b$ initio calculations that show that this effect can be of the same order as the charge-Seebeck effect, using magnetic tunnel junctions (MTJs), where two ferromagnets are separated by a thin insulating tunnel barrier. The effect is related to a half-metallic behaviour of the tunnel junction with respect to the tunnelling states. Our experiments show that a thermoelectric power can be generated in such nanostructures over distances of only $2.1 \mathrm{~nm}$, the thickness of the tunnel barrier. The change from parallel to antiparallel electrode configuration is $-8.7 \mu \mathrm{V} \mathrm{K}^{-1}$ at room temperature, while maintaining all other conditions in the junction constant. Related to this magnetization switching we calculated a magneto-Seebeck effect of $8.8 \%$. In theory, this change is predicted to be up to $100 \mu \mathrm{V} \mathrm{K}^{-1}$, corresponding to $1,000 \%$ (ref. [36]). In future spincaloritronic ${ }^{5}$ applications, the local cooling of an individual nanometre-sized area could, therefore, be switched magnetically. The junction size enables stacking and nano-integration of these thermopower devices.

The magneto-thermal effect is based on the seminal work described in ref. [4]. This gave a general description of the mechanisms that affect a ferromagnetic material when a heat flow causes a temperature gradient. Strong thermomagnetic effects can be expected in a half-metal, where the spin polarization can be up to $100 \%$ (ref. [45]). We can define a spin-dependent Seebeck coefficient by replacing the charge-dependent Seebeck voltage by a voltage generated for each spin channel. The difference between the two spin-dependent Seebeck coefficients is driving a spin accumulation. In contrast, the magneto-Seebeck effect is different from the spin-Seebeck effect, because it is not related to a spin-voltage generation. It occurs in junctions and is similar to the giant and tunnelling magnetoresistance (TMR). It results in a charge-Seebeck effect that is changed by the magnetic orientation of the electrodes. This voltage is accessible directly without conversion. To have a high charge-Seebeck effect, a high asymmetry in the energy dependence with respect to the electrochemical potential for the transport states is necessary, realized in semiconductors as shown in Fig. 2.1a. Consequently, for the thermomagnetic effect, these energy asymmetries must be different for spin-up and spin-down carriers. For our experiments, the recent progress in giant TMR junctions enabled us to use MTJs with high spin asymmetry. Their large contrast in the spin-dependent transmission due to different symmetries of the tunnelling states in the two spin channels should lead also to different energy asymmetries of the tunnelling states as shown in Fig. 2.1b. We define the magneto-Seebeck ratio $\left(S_{\mathrm{MS}}\right)$ from the Seebeck coefficients in the parallel $\left(S_{\mathrm{P}}\right)$ and anti-parallel $\left(S_{\mathrm{AP}}\right)$ configurations:

$$
S_{\mathrm{MS}}=\frac{S_{\mathrm{P}}-S_{\mathrm{AP}}}{\min \left(S_{\mathrm{P}}, S_{\mathrm{AP}}\right)}
$$


At first glance, it seems that the magnetoresistance, the spin-Seebeck effect and the magneto-Seebeck effect should be related to each other. However, these are different effects, and, in general, it is not possible to calculate one from the others.

To understand this point, it is important to realize that the transport coefficients are calculated from the transmission function $T(E)$ of the tunnel junction but that they have different integral values. The conductance $g$ is determined by the integral of the transmission function $T(E)$ multiplied by the derivative of the electron
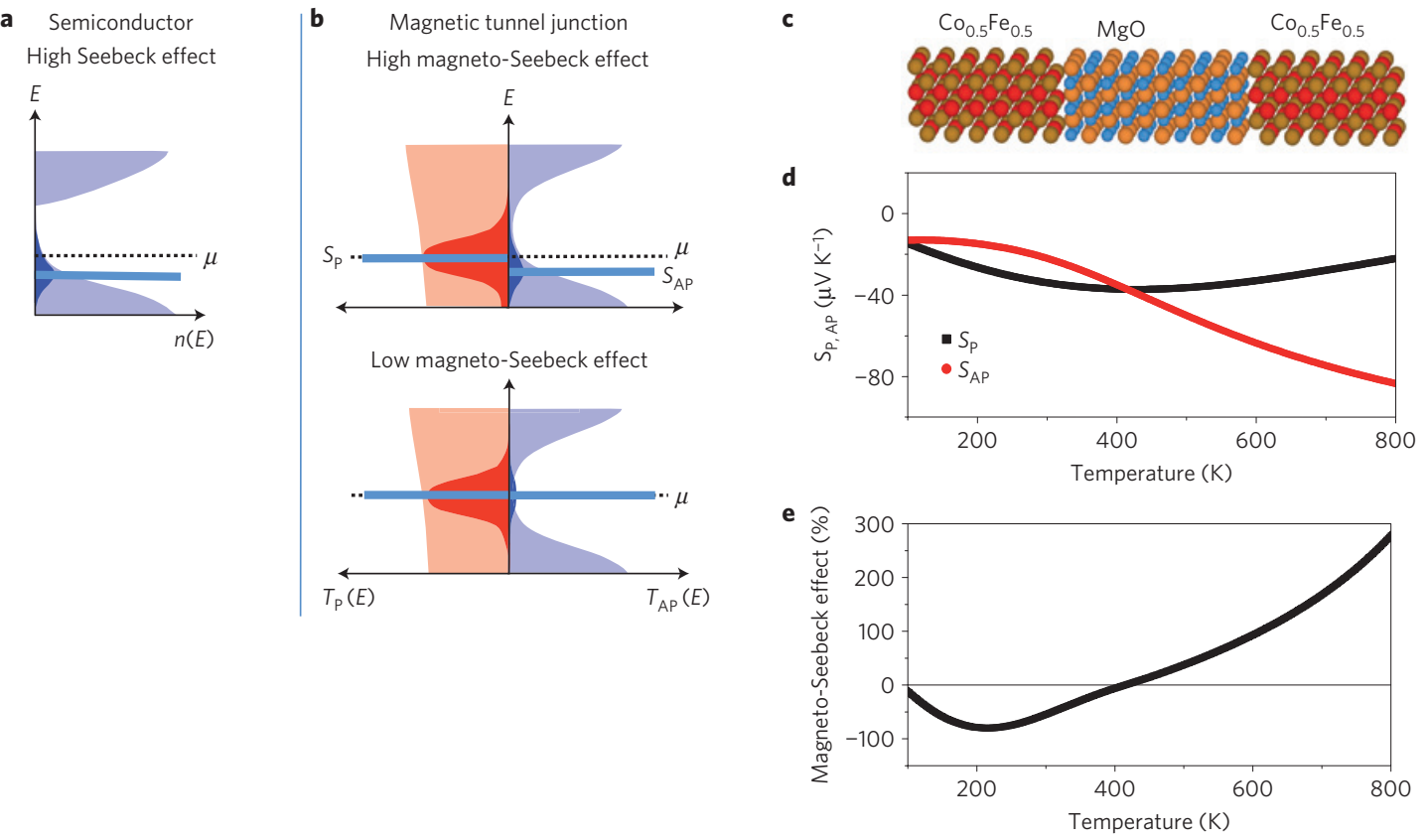

Figure 2.1: Origin of the magneto-Seebeck effect. a, Semiconductors are known to generate high Seebeck effects. b, In MTJs, thermal differences in the electron distributions and strong asymmetry in the spin-dependent tunnelling channels are depicted. $T(E)$ is the transmission of the full tunnel junction, for which either the ferromagnetic electrodes can be a highly spin-polarized half-metal or the combination of the barrier and the ferromagnet exhibits half-metallic characteristics. The function $T(E)\left(-\partial_{E} f(E, \mu, T)\right)$ is given in darker colour. The thick line marks the resulting value of the geometric centre $S_{\mathrm{P}}$ and $S_{\mathrm{AP}}$. In the lower symmetric case, the magneto-Seebeck effect is vanishing. c, Calculation of the Seebeck coefficients as a function of temperature for tunnel junctions with ten monolayers of $\mathrm{MgO}$ as a barrier. The magnetic layers are 20 monolayers thick. The semi-infinite leads are $\mathrm{Cu}$ in the bcc-Fe structure. We assume a mixed termination of FeCo at the $\mathrm{FeCo} / \mathrm{MgO}$ interface that is an ordered, $2 \times 1$, in-plane supercell with one Fe and one Co atom. d, Seebeck coefficients for the parallel configuration and the antiparallel configuration are shown. e, The corresponding magneto-Seebeck effect $S_{\text {MS }}$. 
occupation function $\partial_{E} f(E, \mu, T)$ at temperature $T$ and electrochemical potential $\mu$ :

$$
g=\frac{e^{2}}{h} \int T(E)\left(-\partial_{E} f(E, \mu, T)\right) \mathrm{d} E
$$

The Seebeck coefficient is also given by the transmission function $T(E)$ multiplied by the derivative of the occupation function $\partial_{E} f(E, \mu, T)$ :

$$
S=-\frac{\int T(E)(E-\mu)\left(-\partial_{E} f(E, \mu, T)\right) \mathrm{d} E}{e T \int T(E)\left(-\partial_{E} f(E, \mu, T)\right) \mathrm{d} E}
$$

In contrast to the magnetoresistance, the Seebeck coefficient is the geometric centre of $T(E)\left(-\partial_{E} f(E, \mu, T)\right)$. Figure 2.1b illustrates these quantities for two different cases. The geometric centre for parallel and antiparallel configurations $\left(S_{\mathrm{P}}\right.$ and $S_{\mathrm{AP}}$ ) is marked by the thick line. We assume a transmission function that has different energy asymmetries in both magnetic configurations and different positions of the electrochemical potential. In the first case, a high TMR and a high magnetoSeebeck ratio are obtained. In the second case, the value of $S_{\mathrm{MS}}$ is essentially zero, but the TMR is highest. Generally speaking, cases with vanishing value of $S_{\text {MS }}$ and large TMR (or vice versa) are also possible. Therefore, we can tailor MTJs to be good candidates for large magneto-Seebeck effects. Consequently, we investigated temperature-induced voltages in MTJs starting with samples showing large TMR ratios. Two different types of junction with large TMR values could be used, that is, $\mathrm{Fe}-\mathrm{Co} / \mathrm{MgO} / \mathrm{Fe}-\mathrm{Co}$ and half-metallic compounds. We focus on the former case, as it is demonstrated to have the largest experimental value, $604 \%$ at room temperature ${ }^{15}$. The tunnelling states of the electrons have been thoroughly investigated for MgO-based MTJs and the understanding of spin polarization of the current and the quantitative approach to magnetoresistance in tunnel junctions has advanced enormously in recent years.

Our theoretical investigations are $a b$ initio calculations based on density functional theory. In particular, we used the Korringa-Kohn-Rostoker and the nonequilibrium Green's function method to obtain the transmission function $T(E)$ (ref. [46]). Using $T(E)$, we calculated the transport coefficients according to equations (2.2) and (2.3) (refs [21, 22]). We investigated the magneto-Seebeck coefficients for different temperatures for $\mathrm{Fe}_{0.5} \mathrm{Co}_{0.5} / \mathrm{MgO} / \mathrm{Fe}_{0.5} \mathrm{Co}_{0.5}$ MTJs with bcc structure of the ferromagnetic electrodes. The temperature dependence is considered only within the electron-occupation function. Owing to coherent tunnelling, the atomic structure of the interface could be important. Therefore, we investigated the Seebeck coefficients for different possible interface structures, that is, the Fe-terminated structure, the Co-terminated structure and a mixed-termination structure. The results at a temperature of $300 \mathrm{~K}$ listed in Table 2.1 show a strong dependence on the interface structure. Even a sign change was observed. However, the case where the layer next to the barrier is pure Co or pure Fe is unlikely in the experiment. Consequently, we continued our investigation with the 
Table 2.1: The Seebeck coefficients for parallel $S_{\mathrm{P}}$ and antiparallel $S_{\mathrm{AP}}$ configurations and the magneto-Seebeck effects calculated for different supercells at a temperature of $300 \mathrm{~K}$.

\section{$\mathrm{FeCo} / \mathrm{MgO} / \mathrm{FeCo}$ with a ten-monolayer $\mathrm{MgO}$ barrier}

\begin{tabular}{lcccc}
\hline \hline & $\boldsymbol{S}_{\mathbf{P}}\left(\frac{\mathbf{\mu V}}{\mathbf{K}}\right)$ & $\boldsymbol{S}_{\mathbf{A P}}\left(\frac{\mathbf{\mu V}}{\mathbf{K}}\right)$ & $\boldsymbol{S}_{\mathbf{P}}-\boldsymbol{S}_{\mathrm{AP}}\left(\frac{\boldsymbol{\mu V}}{\mathbf{K}}\right)$ & $\boldsymbol{S}_{\mathrm{MS}}(\%)$ \\
\hline $\mathrm{CoFe}$ & -19.7 & -32.4 & 12.7 & 64.1 \\
$\mathrm{FeCo}$ & 45.9 & -50.0 & 95.9 & 209.0 \\
$\mathrm{CFFC}$ & 9.4 & -44.6 & 54.0 & 573.2 \\
$\mathrm{Co}_{0.5} \mathrm{Fe}_{0.5}$ & -34.0 & -21.9 & -12.1 & -55.2 \\
$\begin{array}{l}\text { Experimental } \\
\text { value }\end{array}$ & $-107.9(-1,300)$ & $-99.2(-1,195)$ & $-8.7(-105)$ & $-8.8(-8.8)$ \\
\hline \hline
\end{tabular}

The results show the sensitivity to the interface composition. $S_{\mathrm{MS}}$ defines the relative change and can be negative or positive. Abbreviations: $\mathrm{CoFe}-\mathrm{Co}_{0.5} \mathrm{Fe}_{0.5}$ layers with $\mathrm{Co}$ at the $\mathrm{MgO}$ interface. $\mathrm{FeCo}-\mathrm{Co}_{0.5} \mathrm{Fe}_{0.5}$ layers with $\mathrm{Fe}$ at the $\mathrm{MgO}$ interface. $\mathrm{CFFC}-\mathrm{Co}_{0.5} \mathrm{Fe}_{0.5}$ layers with $\mathrm{Fe}$ at one of the $\mathrm{MgO}$ interfaces and $\mathrm{Co}$ at the other. $\mathrm{Co}_{0.5} \mathrm{Fe}_{0.5}$ - supercell in plane with $\mathrm{Co:Fe}$ 1:1 at the interface. The values derived from the experiment are given for a temperature difference at the $\mathrm{MgO}$ barrier of $53 \mathrm{mK}(4.4 \mathrm{mK})$ respectively. The temperature difference $\Delta T$ is taken from the numerical simulation of the temperature gradients using the thin-film value (bulk value) of the thermal conductivity of $\mathrm{MgO}$.

mixed-termination structure $\left(\mathrm{Co}_{0.5} \mathrm{Fe}_{0.5}\right)$. In Fig. $2.1 \mathrm{~d}, S_{\mathrm{P}}$ and $S_{\mathrm{AP}}$ are plotted as a function of temperature for a tunnel junction that has an $\mathrm{MgO}$ barrier that was 10 monolayers thick. In addition, we plot the corresponding magneto-Seebeck ratios (Fig. 2.1e). Although $S_{\mathrm{P}}$ and $S_{\mathrm{AP}}$ do not change sign, $S_{\mathrm{MS}}$ does when $S_{\mathrm{P}}=S_{\mathrm{AP}}$. We found that $S_{\mathrm{P}}$ and $S_{\mathrm{AP}}$ were large when compared with charge-Seebeck coefficients.

For the experiments, we use $\mathrm{Co}-\mathrm{Fe}-\mathrm{B} / \mathrm{MgO} / \mathrm{Co}-\mathrm{Fe}-\mathrm{B}$ pseudo-spin-valve structures. The $1 \times 1 \mu^{2}$ tunnel junction is heated homogeneously by $30 \mathrm{~mW}$ laser power (diode laser with 15-20 $\mathrm{mm}$ focus in diameter and a wavelength of $784 \mathrm{~nm}$, Fig. 2.2a,b) and the charge-Seebeck voltage (Seebeck voltage in the following) is measured for the parallel and antiparallel orientations of the layer magnetization (Fig. 2.2c,d). To obtain the temperature distribution and the time constants for the heat diffusion, we used finite-element simulations. Transmission electron microscopy (TEM) in Fig. 2.3a reveals the device geometry that serves as an input to integrate the heat diffusion equation. To calculate the Seebeck coefficients, we estimate a temperature difference $\Delta T$ at the $2.1 \mathrm{~nm} \mathrm{MgO}$ barrier. For polycrystalline $\mathrm{MgO}$ films with a nanometre grain size, the heat conductance is lower than the bulk value owing to the grain boundaries ${ }^{47}$. The high-resolution TEM in Fig. 2.3a, however, reveals a good crystalline quality of the investigated samples. Nevertheless, the thermal resistance at the $\mathrm{Co}-\mathrm{Fe} / \mathrm{MgO}$ interfaces can have similar effects to the grain boundaries. Therefore, we used both the bulk and the reduced value 


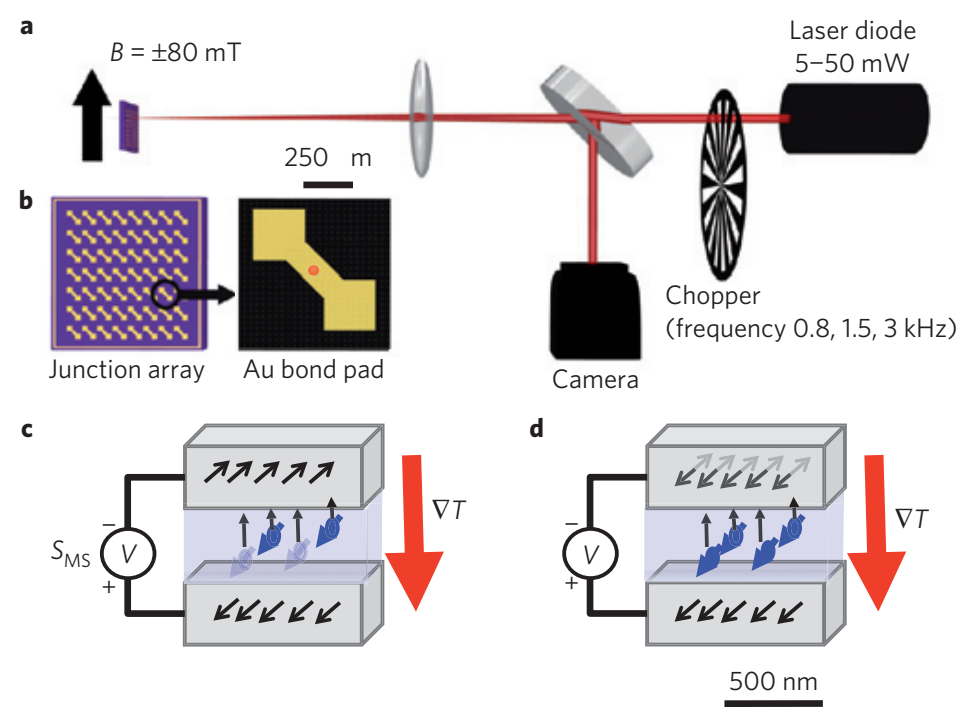

Figure 2.2: Switching of the Seebeck effect through the magnetization. a,b, Schematic representations of the laser-heating set-up (a) and the Au top-contact geometry of the device with the laser spot dimensions (b). c,d, From antiparallel (c) to parallel (d) orientation of the layer magnetization, the charge-Seebeck voltage varies. By the magnitude of its change the magneto-Seebeck effect $S_{\mathrm{MS}}$ is defined.

for the thermal conductivities as given in Supplementary Information.

In Fig. 2.3b we show the resulting temperature profile in a two-dimensional crosssection for $200 \mathrm{ps}$ and $1 \mu \mathrm{s}$ after the laser power is turned on. A series enables determination of the timescales of the heating: the static temperature profile is reached after about $2 \mu \mathrm{s}$. The final temperature distribution is shown as a line scan in Fig. 2.3c across the tunnel junction. A temperature difference at the $2.1 \mathrm{~nm}$ $\mathrm{MgO}$ barrier of $53 \mathrm{mK}(4.4 \mathrm{mK})$ is derived from the numerical simulation using the thin-film value (bulk value) of the thermal conductivity of $\mathrm{MgO}$ respectively.

Figure 2.4 shows the magneto-Seebeck effect of a single MTJ with a TMR of $150 \%$. The temporal voltage traces in Fig. 2.4a, as observed in several junctions, show a peak-like voltage when the laser heating is increased and decreased periodically. A negative peak occurs when the laser power is turned on. From the time constants simulated we identify this voltage peak with the Seebeck voltage generated at the junction. The shutter moving through the laser spot limits the timescale to approximately 10-100 us in our data. As a reference the sequence of the measured laser power is given for each signal trace. The voltage reverses sign when the laser heating is turned off. The Au pads efficiently conduct the heat away from the heat spot (extension of $17.5 \mu \mathrm{m}$ ) into the large bond pads that act as a heat sink. The other side of the junction is then still at a higher temperature and the temperature gradient is reversed. In the lowest curve with an $800 \mathrm{~Hz}$ modu- 

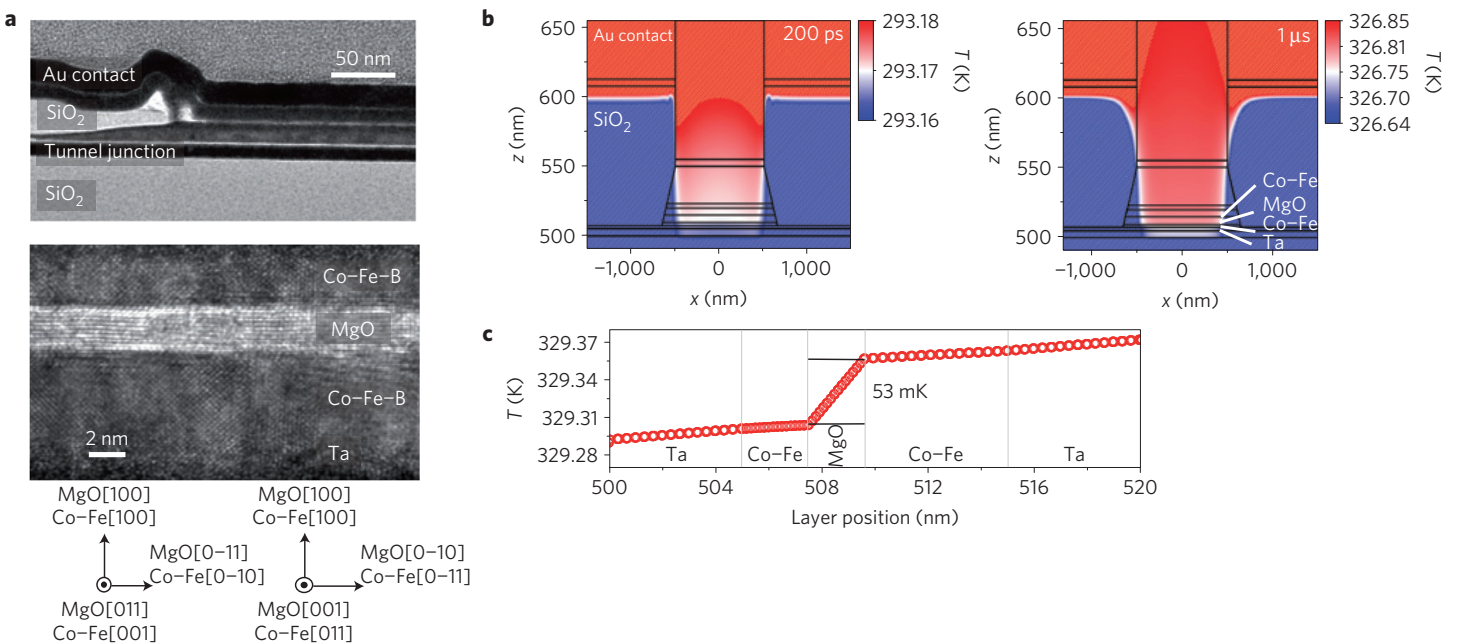

Figure 2.3: Cross-sections and temperature gradients in the tunnel junction. a, TMR junctions: device structure studied with TEM. The high resolution shows the epitaxial relationship Fe- $\mathrm{Co}(001) / \mathrm{MgO}(001)$ for two transmission directions, $\mathrm{MgO}[100]$ and $\mathrm{MgO}[110]$. b, The simulated temperature distributions for $200 \mathrm{ps}$ and $1 \mu \mathrm{s}$ after the laser power of $30 \mathrm{~mW}$ is turned on in a two-dimensional crosssection. c, The temperatures for the final static-equilibrium condition as a line scan.

lation frequency, the MTJ was heated asymmetrically, enabling a longer cooling time. The Seebeck voltages for the parallel and antiparallel configurations are determined from Fig. 2.4b, in which the Seebeck voltage is shown as a function of the applied field. As expected from the temporal traces, a larger value was found for the fastest modulation of the laser power (at $3 \mathrm{kHz}$ ). The Seebeck voltage at the junction contributes more to the total signal than it does for the slower modulation, where the whole sample heats up on a larger area. A signal proportional to the modulation frequency is the dominant component. We obtain $-5.7 \mu \mathrm{V}$ for the parallel and $-5.3 \mu \mathrm{V}$ for the antiparallel orientation, that is, a change of the Seebeck voltage by $0.4 \mu \mathrm{V}$ for the tunnel junction. Fluence-dependent experiments suggest that the increase of the Seebeck voltage with laser power depends on the increase of the temperature gradient at the barrier and the base temperature at the junction, which is increased by the laser power as well, as discussed further in Supplementary Information. If the junction barrier is pushed through a dielectric breakdown ${ }^{48}$, the magneto-Seebeck effect disappears.

The experimental results and the theoretical predictions for the Seebeck coefficients are summarized in Table 2.1. The theoretical prediction for the $\mathrm{Co}_{0.5} \mathrm{Fe}_{0.5}$ case with Co:Fe 1:1 at the interface is closest to the experiment. The values are negative for both the parallel and the antiparallel configuration. To calculate the 

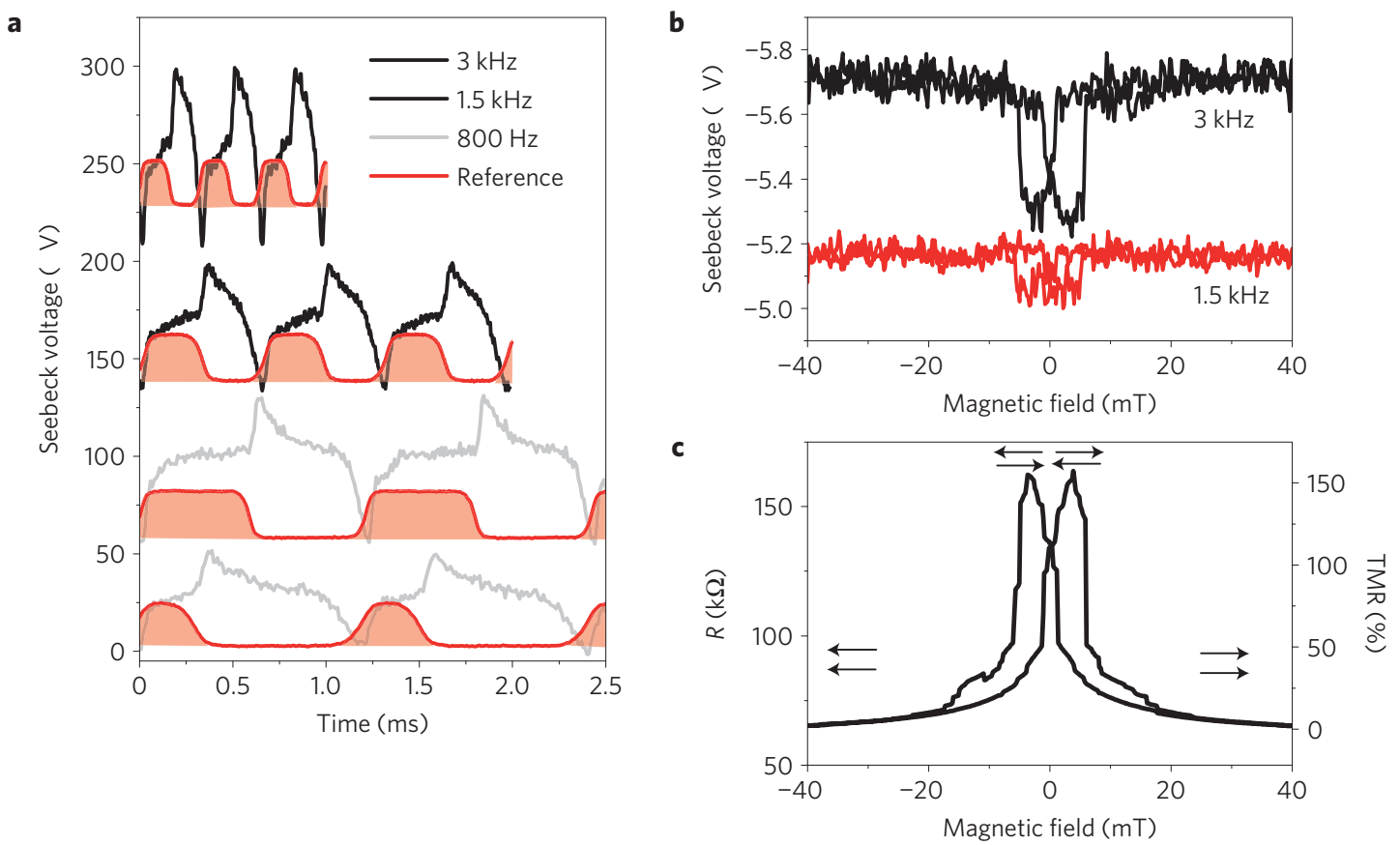

Figure 2.4: Seebeck voltages for Fe-Co-B/MgO/Fe-Co-B elements. a, Temporal traces are shown for different modulation frequencies. The heating by the laser is shown in red overlaid on the corresponding Seebeck voltage (the voltages are shifted for clarity). b, Magneto-Seebeck voltages are given above for 1.5 and $3 \mathrm{kHz}$ lock-in modulation. c, The corresponding magnetoresistance (TMR) shows a hard-soft switching of the pseudo-spin-valve structure.

Seebeck coefficients from the experimentally determined Seebeck voltage $V_{\mathrm{P}, \mathrm{AP}}$, we take the temperature gradient to be $53 \mathrm{mK}$ across the $2.1 \mathrm{~nm} \mathrm{MgO}$ tunnel barrier from our numerical modelling. Thus, we obtain a value of $V_{\mathrm{P}} / \Delta T=-108 \mu \mathrm{V} \mathrm{K} \mathrm{K}^{-1}$ for the Seebeck coefficient $S_{\mathrm{P}}$ for the parallel orientation. A decrease of the thermal gradient taking the bulk value for the $\mathrm{MgO}$ thermal conductivity as input parameter in our model increases the Seebeck coefficients calculated accordingly. This enables derivation of an upper limit of $-1,300 \mu \mathrm{V} \mathrm{K}^{-1}$ for the Seebeck coefficient, given in brackets. Note that spurious other voltages generated in the layer stacks or within the heated device change the Seebeck-effect amplitude, but not the difference of the Seebeck voltage for parallel and antiparallel configurations. In accordance with this, the experimental results for the difference $\left(S_{\mathrm{P}}-S_{\mathrm{AP}}\right)$ of $-8.7 \mu \mathrm{V} \mathrm{K}^{-1}$ are closer to the predicted value of $-12.1 \mu \mathrm{VK}^{-1}$ than the individual values of $S_{\mathrm{P}}$ and $S_{\mathrm{AP}}$. The lower limit of the magneto-Seebeck effect, that is, the relative change of the Seebeck voltage for the parallel and the antiparallel case, is $-8.8 \%$.

Finally, the magneto-Seebeck effect in MTJs enables control of these effects. As a major strategy to develop the possibilities opening up with the magneto-Seebeck 
effect it is crucial to tailor the thermal tunnelling current arising from the majority and the minority spins, that is, to maximize the shift of the geometric centre relative to the Fermi level of these electronic states contributing to the thermal transport. The calculations demonstrate that even the sign of the magneto-Seebeck effect can be controlled using different Co-Fe compositions. Findings on tunnel junctions using a different method (resistive heating) yielding the same effect magnitudes with different sign were recently reported ${ }^{40}$ using $\mathrm{Co}-\mathrm{Fe}-\mathrm{B} / \mathrm{MgO} / \mathrm{Co}-\mathrm{Fe}-\mathrm{B}$ devices but of different composition and structure (using a Singulus Tech. cluster tool). The qualitative change for the devices presented here however enables comparison of theoretical and experimental results. The results presented compare well to the theoretically predicted change, including the predicted sign reversal of the magnetoSeebeck effect at elevated temperatures (see Supplementary Information). Further, the experiments showed that the magneto-Seebeck effect can be generated over length scales of only a few nanometres - across a 2.1-nm-thick tunnel barrier in our case. This reveals that the magneto-Seebeck effect in MTJs can be used to manipulate and design thermovoltages in nanometre-scale devices. The contrast for switching the voltage can be increased further in the future, which will enable control of the Seebeck effect by magnetic switching.

Note added in proof. After acceptance of this paper, we became aware of a paper by Jansen and colleagues ${ }^{35}$ demonstrating a thermal injection of spins from a ferromagnet through an $\mathrm{Al}_{2} \mathrm{O}_{3}$ tunnel barrier into silicon.

\subsection{Methods}

\subsubsection{Fabrication}

The Co-Fe-B films were prepared by magnetron sputtering using 2 inch targets with compositions of $\mathrm{Co}_{0.4} \mathrm{Fe}_{0.4} \mathrm{~B}_{0.2}$ (analysis $\mathrm{Co}$ :Fe 0.52:0.48)and $\mathrm{Co}_{0.2} \mathrm{Fe}_{0.6} \mathrm{~B}_{0.2}$ (analysis $\mathrm{Co}: \mathrm{Fe}$ 0.32:0.68) in an ultrahigh-vacuum system with a base pressure of $5 \times 10^{-10}$ mbar. They are annealed ex situ at temperatures of $450-550{ }^{\circ} \mathrm{C}$ (postgrowth annealing, 20-60 min). For samples prepared in the Göttingen chamber $\mathrm{MgO}$ was e-beam evaporated after transferring to a separate ultrahigh-vacuum chamber with base pressure of $5 \times 10^{-10}$ mbar (maximum TMR reached is $200 \%$ at room temperature). With the Bielefeld chamber, $\mathrm{MgO}$ was prepared by magnetron sputtering (maximum TMR reached is $330 \%$ at room temperature). The sample stack was reduced to a simple pseudo-spin-valve structure to minimize the contribution of spurious Seebeck voltages at metal interfaces not stemming from the junction: $\mathrm{Au} 27 \mathrm{~nm} / \mathrm{Ru} 3 \mathrm{~nm} / \mathrm{Ta} 5 \mathrm{~nm} / \mathrm{Co}-\mathrm{Fe}-\mathrm{B} 5.4 \mathrm{~nm} / \mathrm{MgO} 2.1 \mathrm{~nm} / \mathrm{Co}-\mathrm{Fe}-\mathrm{B}$ $2.5 \mathrm{~nm} / \mathrm{Ta} 5 \mathrm{~nm} / \mathrm{SiO}_{2} 500 \mathrm{~nm} / \mathrm{Si}(100)$. This was done as a trade-off with the magnetic separation of the switching fields, because no antiferromagnetic exchange-bias layer is used, which could also give a magnetic contribution. After ex situ annealing in a constant field, further structuring was done by standard ion-beam etching to 
yield $1 \times 1 \mu^{2}$ to $12.5 \times 12.5 \mu^{2}$ junctions to the $\mathrm{MgO}$ barrier. The high-resolution TEM data in Fig. 2.3 (bottom, left) reveal the coherent growth of crystallized Co$\mathrm{Fe}(110)$ on each side of the $\mathrm{MgO}(100)$ barrier (solid-state epitaxy) in columns that can be identified $(\mathrm{MgO}[001]$ and $\mathrm{MgO}[110]$ in the transmission direction). As an isolation layer at the sides of the element, a 100 -nm-thick $\mathrm{SiO}_{2}$ layer was grown by thermal evaporation. A 100-nm-thick, top-contact Au layer was deposited as a bond pad. This also prevents direct optical carrier excitation in the $\mathrm{MgO}$ barrier. A $5 \mathrm{~nm}$ layer of $\mathrm{Cr}$ was deposited below the Au top-contact layer for better adhesion on the $\mathrm{SiO}_{2}$ isolation.

\subsubsection{Experimental set-up}

For the laser heating, a $100 \mathrm{~mW}$, Toptica, intensity-stabilized laser diode module (wavelength, $\lambda=784 \mathrm{~nm}$ ) was focused to a diameter of $15-20 \mu \mathrm{m}$ full-width at halfmaximum. For the standard experiments we used $30 \mathrm{~mW}$ laser power. The beam position on top of the bond pad was controlled through a camera. The intensity was modulated using $800 \mathrm{~Hz}, 1.5 \mathrm{kHz}$ and $3 \mathrm{kHz}$ modulation frequencies. To prevent a current flow in the system that could be modified by the change of the resistance of the junction, a high-input-impedance (100 G $\Omega$ ) LT1113 precision operational amplifier (Linear Technology) was used. The bandwidth of the amplifier is $5 \mathrm{MHz}$. This was installed close to the sample to minimize the effect of the cable capacitance $(<15 \mathrm{pF})$. A simulation of the circuit with the sample resistance showed that the change of the resistance will contribute $<1 \mathrm{nV}$ to the absolute voltage. Curves of Seebeck voltage versus magnetic field are measured using a Stanford Linear Research lock-in amplifier.

\subsubsection{Thermal modelling of parameters (COMSOL)}

To simulate heat flow and temperature distribution, the MTJ was modelled using the COMSOL finite-element package. The tunnel-junction geometry was taken from the cross-sectional TEM data as input parameters. The element was embedded into a $3 \mu \mathrm{m}$ cylinder. The heat flow from the laser heating comes from the top. For a $30 \mathrm{~mW}$ laser power the absorbed laser power is $10 \mathrm{~mW}$. The temperature at the bottom of the cylinder in the $\mathrm{Si}(100)$ substrate was set to ambient temperature. The $500 \mathrm{~nm} \mathrm{SiO}_{2}$ layer on top of the substrate is the bottleneck for heat diffusion through the cylinder stack. The temperature at the bottom layer of the element depends sensitively on the heat flow though the $\mathrm{SiO}_{2}$ layer and determines the $2 \mu \mathrm{s}$ needed to reach the final heat gradient. A prism-shaped, adaptive mesh was used with resolution $>10 \mathrm{~nm}$ in the plane and subnanometre perpendicular to the plane. In addition, we carried out simulations on a larger length scale to simulate lateral heat diffusion. The heated area extends to about $17.5 \mu \mathrm{m}$ diameter, and, in this case, the absolute temperature increase is reduced to $8 \mathrm{~K}$. The equilibrium heat 
gradient is attained within about $2 \mu \mathrm{s}$. For the $2.1 \mathrm{~nm} \mathrm{MgO}$ thin tunnel barrier, a value of the thermal conductance of $\kappa=4 \mathrm{~W}(\mathrm{mK})^{-1}$ is assumed to be closest to reality. This value has been determined experimentally for a thin film ${ }^{47}$. It is expected to be much closer to the bulk value of $\kappa=48 \mathrm{~W}(\mathrm{mK})^{-1}$, which gives an upper limit for the Seebeck coefficient. All material parameters used in the numerical model are provided in a table in Supplementary Information.

\subsection{Acknowledgements}

A.T. acknowledges the Ministry of Innovation, Science and Research of the North Rhine-Westphalia state government for financial support. M.M., M.S., M.W. and P.P. acknowledge the funding provided by the German Research Foundation through the SFB 602 for the TEM work. M.C., M.B. and C.H. acknowledge support from German Research Foundation SPP 1386 and German Research Foundation grant HE 5922/1-1. J.S.M acknowledges support by the US National Science Foundation and Office of Naval Research. We acknowledge A. Zeghuzi's help for extra COMSOL calculations. This work was initiated by the SpinCaT priority program.

\subsection{Author contributions}

M.W. and J.W. carried out experiments; M.W., V.Z., M.Sch. and D.E. characterized and prepared the TMR devices; P.P. and M.S. carried out the high-resolution TEM; M.W., J.W. and M.M. analysed the data; M.W. carried out the COMSOL calculations; M.C., M.B. and C.H. did the $a b$ initio transport calculations; A.T. and M.M. designed the research approach; C.H., M.M. and A.T. wrote the manuscript and developed the model; M.W., J.S.M., A.T., M.M. and C.H. contributed to the development of the experiments; G.R., J.S.M., A.T., M.M., C.H. and all authors discussed the experiments and the manuscript.

\subsection{Additional information}

The authors declare no competing financial interests. Supplementary information accompanies this paper on www.nature.com/naturematerials. Reprints and permissions information is available online at http://www.nature.com/reprints. Correspondence and requests for materials should be addressed to M.M. 



\title{
Chapter 3
}

\section{Time-resolved measurement of the tunnel magneto-Seebeck effect in a single magnetic tunnel junction}

\author{
A. Boehnke, M. Walter, N. Roschewsky, et al. \\ Rev. Sci. Instrum. 84, 063905 (2013) \\ DOI: $10.1063 / 1.4811130$
}

\begin{abstract}
Recently, several groups have reported spin-dependent thermoelectric effects in magnetic tunnel junctions. In this paper, we present a setup for time-resolved measurements of thermovoltages and thermocurrents of a single micro- to nanometer-scaled tunnel junction. An electrically modulated diode laser is used to create a temperature gradient across the tunnel junction layer stack. This laser modulation technique enables the recording of time-dependent thermovoltage signals with a temporal resolution only limited by the preamplifier for the thermovoltage. So far, time-dependent thermovoltage could not be interpreted. Now, with the setup presented in this paper, it is possible to distinguish different Seebeck voltage contributions to the overall measured voltage signal in the $\mu$ s time regime. A model circuit is developed that explains those voltage contributions on different sample types. Further, it will be shown that a voltage signal arising from the magnetic tunnel junction can only be observed when the laser spot is directly centered on top of the magnetic tunnel junction, which allows a lateral separation of the effects.
\end{abstract}

\subsection{Introduction}

In recent years, the research field "spin caloritronics" has attracted considerable attention in the magnetism and spintronics communities. ${ }^{5,11}$ New spin-dependent thermoelectric effects have been discovered in ferromagnetic metals, ${ }^{10}$ insulators, ${ }^{25}$ and semiconductors. ${ }^{26}$ Triggered by the experiments of Gravier et al. ${ }^{8}$ and Shi et al. ${ }^{6}$ on giant magneto resistance (GMR) multilayers, and by theoretical predictions of 
large magnetothermoelectric effects in magnetic tunnel junctions, ${ }^{36}$ several groups reported observations of a tunnel magneto-Seebeck effect (TMS) in magnetic tunnel junctions (MTJs) with MgO- ${ }^{40,49}$ and alumina-barriers. ${ }^{37}$ A closely related effect is thermal spin injection into silicon through Seebeck spin tunneling. ${ }^{35}$ In non-local spin valves, thermally driven spin injection was discovered ${ }^{23}$ and Peltier and Seebeck effects were studied. ${ }^{34}$ The number of these new effects, combined with the proposed thermal spin-transfer torque ${ }^{44,50}$ might enable the fabrication of thermally driven Magnetoresistive Random Access Memory (MRAM) and other spintronic devices.

Some of the effects are vividly discussed in the community. ${ }^{28-33,51}$ In contrast, the experiments on $\mathrm{CoFeB} / \mathrm{MgO}$-based MTJs with high tunnel magnetoresistance (TMR) ratios, in which either a laser ${ }^{49}$ or resistive heating ${ }^{40}$ is used to generate the temperature gradients, show comparable results. These are of the same magnitude as predicted by ab initio calculations. ${ }^{36,49}$ For MTJs with alumina-barrier, larger Seebeck voltages as compared to $\mathrm{MgO}$-barriers at comparable temperature gradients are found. However, there are a few variations between the different experiments: First, the sign of the Seebeck voltage remains unclear, which could also vary depending on temperature and Co-Fe-composition. ${ }^{52}$ Further, it was reported by Ref. [37] that no magnetic effect is observed in the thermocurrent obtained with alumina-barriers and that Seebeck voltages could be observed when heating the electrical leads a distance of the order of millimeters away from the MTJ.

In the following, we will address these issues and show results for the Seebeck voltage as well as for the thermocurrent and the determination of the voltage sign with a lock-in technique in Sec. 3.4. A model circuit is developed in Sec. 3.5 to interpret the time-dependent signals. In Sec. 3.6 heating-position dependent measurements are presented, which reveal that the Seebeck voltage is generated locally at the MTJ in this geometry.

\subsection{Description of the experimental setup}

The setup used in this work is based on the experiments performed by Gravier et $a .^{7}$ on metallic nanowires. We adapted the electrical and optical techniques to the requirements for measuring small thermovoltages across a micrometer-sized single MTJ.

To heat the MTJ from the top and to create a temperature gradient across the layer stack, a $150 \mathrm{~mW}$ laser diode (Toptica ibeam-smart-640-s) is focused down to a beamwaist of $5 \mu \mathrm{m}-10 \mu \mathrm{m}$ using a microscope objective (Mitutoyo M Plan Apo $10 \mathrm{x})$. The central laser wavelength is $637 \mathrm{~nm}$. An exact positioning of the laser spot onto the MTJ is crucial for obtaining reliable voltage measurements. Thus, the position of the laser spot can be controlled using a confocal microscope as depicted in Fig. 3.1(a). With a set of different electromagnets, the sample can be studied in magnetic fields $B_{\text {ip }} \leq 250 \mathrm{mT}$ in-plane and $B_{\mathrm{pp}} \leq 150 \mathrm{mT}$ perpendicular to plane. 
(a)

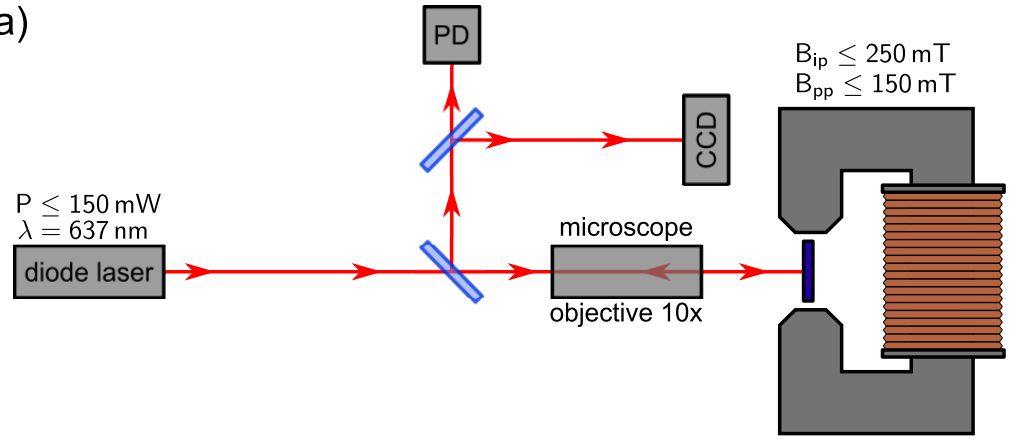

(b)

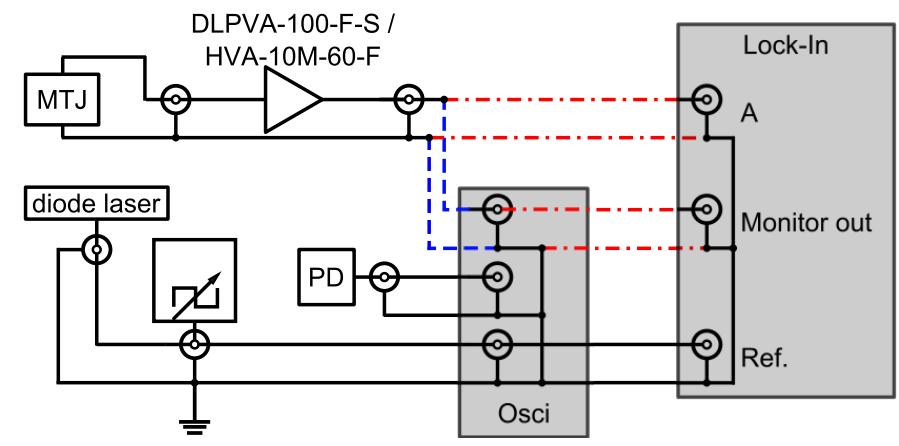

Figure 3.1: Experimental setup for measurements of the TMS effect: (a) Optical setup with confocal microscope including a fast photodiode (PD) and a CCD camera, (b) electrical setup showing the connections of the sample and optoelectronic components to the lock-in amplifier and oscilloscope.

The thermovoltage is detected with a lock-in amplifier. In our earlier publication, ${ }^{49}$ a mechanical chopper was used to modulate the laser heating at $1.5 \mathrm{kHz}$, however it was found that the beamwaist of the unfocussed laser in combination with the mechanical chopping decreases the time-resolution of the thermovoltage detected by the oscilloscope. As a consequence, a waveform generator (Agilent 33500B) has been implemented to modulate the laser diode power with a square wave of $1.5 \mathrm{kHz}$. As can be seen from Fig. 3.1(a), a fast photodiode (EOT ET-2030, rise time of $<300 \mathrm{ps}$ ) is integrated in the confocal microscope part of the setup to check the square-wave form of the light intensity. With this optical setup a rise and fall time of $<1 \mu$ s of the light intensity can be achieved. This is faster than the rise time of the preamplifier used for high impedance MTJs. Thus, the time-resolution of the measured voltage signal of the MTJs is only limited by the electronic equipment, which is shown in Fig. 3.1(b): The MTJ is connected to a precision voltage preamplifier via shielded cables (total length $1.6 \mathrm{~m}$ ). Depending on the MTJ resistance either a high impedance amplifier (femto DLPVA-100-F-S) with a rise time of $3.5 \mu \mathrm{s}$ or a faster amplifier for low impedance sources (HVA-10M-60-F) with a rise time of $3.5 \mathrm{~ns}$ can be used. The signal preamplified by $60 \mathrm{~dB}-80 \mathrm{~dB}$ is then fed either to 
the oscilloscope directly (blue dashed line in Fig. 3.1(b)) or to the lock-in amplifier (red dashed-dotted line in Fig. 3.1(b)). In the latter case, the time-dependent voltage signals are recorded by the oscilloscope which then is connected to the monitor out of the lock-in amplifier. A Stanford Research Systems SR830 lock-in amplifier and a Philips PM3382 oscilloscope are used. All electrical components are triggered by the waveform generator and carefully grounded to minimize noise coupling into the measurement circuit. The noise level of the setup is within a range of $10 \mathrm{nV}-$ $50 \mathrm{nV}$, which is of the same order as Johnson-Nyquist-noise of the MTJ's resistance at room temperature. The low noise level enables observing small voltage changes on the order of nanovolts at low laser intensities, as shown in Fig. 3.2.

For a thermocurrent measurement, the MTJ is connected without preamplifier to the lock-in amplifier set to current detection.

Table 3.1: Material parameters for COMSOL simulations. If not specified otherwise, the values are taken from Refs. [39, 49, 53, 54]. The thermal conductivities used in the simulations are printed in bold letters. Experimental thin film values are given where available.

\begin{tabular}{|c|c|c|c|}
\hline Material & $\rho\left(10^{3} \frac{\mathrm{kg}}{\mathrm{m}^{3}}\right)$ & $c_{V / p}\left(\frac{\mathrm{J}}{\mathrm{kg} \mathrm{K}}\right)$ & $\kappa_{\text {bulk }} / \kappa_{\text {thin }}^{\exp }\left(\frac{\mathrm{W}}{\mathrm{mK}}\right)$ \\
\hline $\mathrm{Au}$ & 19.32 & 128 & $320.0 / 70^{\mathrm{a}}-170^{\mathrm{b}}$ \\
\hline $\mathrm{Cr}$ & 7.15 & 449 & 94.0 \\
\hline $\mathrm{Ru}$ & 12.37 & 238 & 117.0 \\
\hline $\mathrm{Ta}$ & 16.65 & 140 & 57.0 \\
\hline Permalloy & 8.7 & 460 & 19.0 \\
\hline IrMn & 10.18 & 69.7 & 6.0 \\
\hline Co-Fe-B & 8.22 & 440 & 86.7 \\
\hline $\mathrm{MgO}$ & 3.58 & 935 & $48.0 / \mathbf{4 . 0}^{\mathrm{c}}$ \\
\hline $\mathrm{SiO}_{2}$ & 2.20 & 1052 & 1.4 \\
\hline $\mathrm{Si}$ & 2.33 & 700 & 150.0 \\
\hline $\mathrm{SiN}$ & 3.11 & 700 & 35.9 \\
\hline
\end{tabular}

\subsubsection{Determination of temperatures}

Since the $\mathrm{CoFeB}$ and $\mathrm{MgO}$ layers of only a few nanometer thickness are buried under several layers of electrical leads, it is very difficult to reliably measure the 
temperature difference across them. Putting thermocouples to the electrical leads of the MTJ can only give a rough estimate of the temperature distribution.

Consequently, the heat conduction equation is, therefore, numerically solved using COMSOL Multiphysics ${ }^{57}$ to determine the temperatures of the MTJ layers. The results have to be regarded as estimates, since interface heat resistances are not taken into account and bulk values of thermal conductivities, densities and heat capacities are used for the metals layers. These material parameters are taken from literature shown in Table 3.1 for the MTJ materials. The table shows that the thermal conductivity of an $\mathrm{Au}$ thin film is lower by at least a factor of 2 . In addition, we used the experimentally observed thin film value for the $\mathrm{MgO}$ layer, because here the thermal conductivity changes by an order of magnitude. This improves the reliability of our simulations. ${ }^{47,49}$ Further details on the simulations can be found in earlier publications. ${ }^{49,50}$ The temperature difference across the $1.5 \mathrm{~nm}$ $\mathrm{MgO}$ layer resulting from the simulations is used in section 3.4.1 in combination with the measured voltage to calculate the Seebeck coefficients.

\subsection{Sample preparation}

The MTJs are prepared on two types of substrates: $\mathrm{MgO}$ and oxidized p-type silicon ( $\mathrm{Si})\left(50 \mathrm{~nm} \mathrm{SiO}_{2}\right.$, resistivity of $\left.20 \Omega \mathrm{cm}\right)$ by sputter deposition in a Leybold Vakuum $\mathrm{GmbH}$ CLAB 600. The film system on $\mathrm{MgO}$ consists of bottom contact $\mathrm{Ta} 5 / \mathrm{Ru}$ 30/Ta 10/Ru 5; pinned layer MnIr 15/CoFeB 3; tunnel barrier MgO 1.5; free layer $\mathrm{CoFeB} 3 / \mathrm{NiFe} 6$; top contact $\mathrm{Ta} 3 / \mathrm{Ru} 3 / \mathrm{Ta} 3 / \mathrm{Au} 15$ (thicknesses are given in $\mathrm{nm}$ ). In case of $\mathrm{Si} / \mathrm{SiO}_{2}$ substrates the pinned layer is slightly changed to MnIr $12 / \mathrm{CoFe}$ $3 / \mathrm{Ru} 0.9 / \mathrm{CoFeB} 3$. Elliptical MTJs with a size of $6 \mu \mathrm{m} \times 4 \mu \mathrm{m}$ are produced by electron beam lithography and subsequent ion beam etching. Afterwards, $100 \mathrm{~nm}$ of SiN are sputter deposited next to the MTJs as insulator. An Au bond pad is placed adjacent to the MTJs in an additional sputtering and patterning process for connecting the $15 \mathrm{~nm} \mathrm{Au}$ top contact to the measurement electronics. This allows free optical access to the MTJ.

\subsection{Experiments on magnesium oxide and silicon substrates}

\subsubsection{TMR and TMS measurements}

Thermoelectric effects can be derived theoretically from thermodynamic principles. For the case of an MTJ, the thermoelectric coefficients dependent on the tunneling probability can be expressed by equations based on the Landauer formula. ${ }^{18,21,36,37,58}$ In this way, the influence of spin transport on the Seebeck voltage of an MTJ can be described. To clarify the interconnection between the different transport coefficients and sign conventions, we first derive the Seebeck voltage, Seebeck current, 
TMS and TMR from the thermodynamic kinetic equations and the moments of the transport integral.

The charge transport through the barrier of the MTJ is given as

$$
I=G V+G S \Delta T
$$

where $G$ is the electric conductance and $S$ is the Seebeck coefficient. According to Eq. (3.1), a current $I$ is either generated by an external voltage $V$ or by a temperature gradient $\Delta T$. In a Seebeck current measurement no external voltage is applied to the MTJ $(V=0)$ whereas in a perfect voltage measurement no current is transported in the circuit $(I=0)$, which yields

$$
I=G S \Delta T, \quad V=-S \Delta T
$$

for the measured current and voltage, respectively. The coefficients can be rewritten $\operatorname{as}^{22,36}$

$$
G=e^{2} L_{0}, \quad S=-\frac{1}{e T} \frac{L_{1}}{L_{0}}
$$

using the moments

$$
L_{n}=\frac{2}{h} \int T(E)(E-\mu)^{n}\left[-\partial_{E} f(E, \mu, T)\right] \mathrm{d} E
$$

dependent on $f(E, \mu, T)$, the Fermi occupation function at a given energy $E$, electrochemical potential $\mu$ and temperature $T$, and on the energy-dependent transmission probability $T(E)$ that is different for the parallel $(\mathrm{P})$ and antiparallel (AP) orientation of the bottom and top layer's magnetization, which leads to different moments for both states. ${ }^{36,49}$ Thus, the TMS is calculated analogous to the TMR:

$$
\mathrm{TMR}=\frac{R_{\mathrm{AP}}-R_{\mathrm{P}}}{R_{\mathrm{P}}}, \quad \mathrm{TMS}=\frac{S_{\mathrm{P}}-S_{\mathrm{AP}}}{\min \left(\left|S_{\mathrm{P}}\right|,\left|S_{\mathrm{AP}}\right|\right)} .
$$

The MTJs on $\mathrm{Si} / \mathrm{SiO}_{2}$ and $\mathrm{MgO}$ were prepared to investigate the influence of the substrate material on the TMR and TMS measurements. Fig. 3.2 shows field dependent resistance and Seebeck voltage curves of elliptical MTJs with an area of $19 \mu \mathrm{m}^{2}$ prepared on $\mathrm{Si} / \mathrm{SiO}_{2}$ and $\mathrm{MgO}$, respectively.

In case of the $\mathrm{Si} / \mathrm{SiO}_{2}$ substrate, the resistance of the MTJ switches between $1583 \Omega$ in the antiparallel and $864 \Omega$ in the parallel orientation of the ferromagnetic layers, which yields a TMR ratio of $83 \%$. The Seebeck voltage, generated by laser heating with a power of $10 \mathrm{~mW}$, changes from $1.39 \mu \mathrm{V}$ in the antiparallel to $1.34 \mu \mathrm{V}$ in the parallel state resulting in a TMS ratio of $3.7 \%$. The Seebeck voltage detected by the lock-in amplifier is positive as shown in Fig. 3.4(a). This means that the electrons are accumulated at the cold electrode, which results in a negative Seebeck coefficient (Eq. (3.2)). 


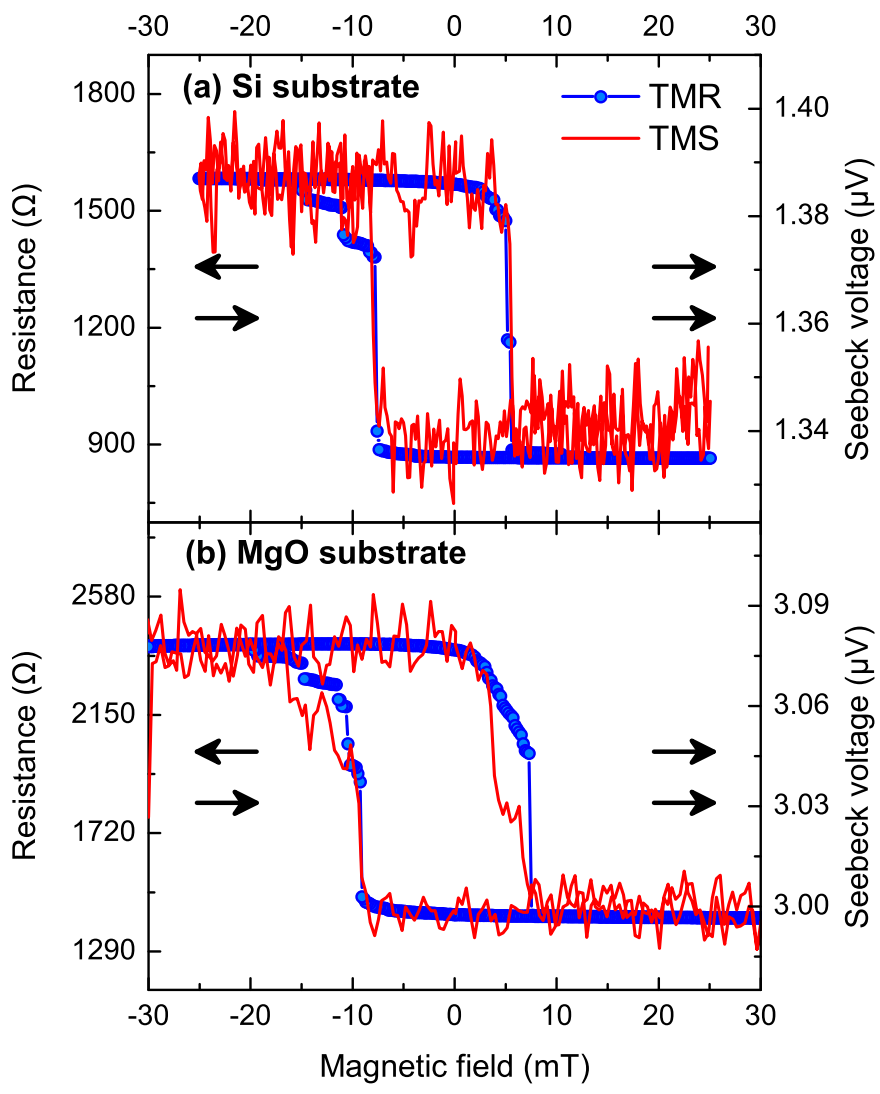

Figure 3.2: Low laser power and different substrates: TMR (blue circles, left scale) and TMS (red line, right scale) of nominally identical MTJs on (a) $\mathrm{Si} / \mathrm{SiO}_{2}$ and (b) $\mathrm{MgO}$ substrates obtained with a laser power of $10 \mathrm{~mW}$ and $15 \mathrm{~mW}$, respectively. The arrows represent the relative orientation of the magnetic layers. The TMR and TMS values are given in Table 3.2.

The MTJ on $\mathrm{MgO}$ exhibits a larger resistance than the MTJ on $\mathrm{Si} / \mathrm{SiO}_{2}$ substrate. The resistance varies between $2400 \Omega$ in the antiparallel and $1411 \Omega$ in the parallel state. A TMR ratio of $70 \%$ is obtained. The corresponding Seebeck voltage, induced by laser heating with $15 \mathrm{~mW}$ laser power, switches at the same magnetic fields between $3.08 \mu \mathrm{V}$ and $3.00 \mu \mathrm{V}$ gaining a TMS ratio of $2.6 \%$. As for the MTJ on $\mathrm{Si} / \mathrm{SiO}_{2}$ substrate, the Seebeck voltage is again positive (Fig. 3.4(b)).

In Table 3.2 the parameters of the TMR and TMS are shown. The small differences are within the normal deviations between different MTJs and can also be due to different growth conditions on the two substrates. As a consequence, no evidence for influence of parasitic Seebeck voltages arising from different substrates on the TMS measurements is found.

The Seebeck coefficients in Table 3.2 are calculated from the above mentioned simulated temperature gradient across the barrier and the thermovoltage generated inside the MTJ. This thermovoltage consists of a spin-dependent component from the ferromagnetic electrodes and a spin-independent background from the other layers in the MTJ. A possible solution to estimate this spin-independent background is given in Ref. [39]: The MTJ is forced to a dielectric breakdown after the TMS measurement is performed and the remaining, spin-independent background ther- 
movoltage is determined. The background thermovoltage is approximately $0.05 \frac{\mu \mathrm{V}}{\mathrm{mW}}$ up to $0.4 \frac{\mu \mathrm{V}}{\mathrm{mW}}$, such that after subtraction, the resulting TMS ratios are around $20 \%$ for the data presented in this paper. However, the morphology of the layers changes due to the voltage stress applied to the MTJ, e.g., the CoFeB can change from an amorphous to a crystalline structure and the interfaces between the thin films can be destroyed. ${ }^{48}$ Therefore, this method allows only an estimation for the background thermovoltages arising from other sources of the layer stack of the tunnel junction that do not contribute to the TMS itself.

Table 3.2: Comparison of TMR and TMS on $\mathrm{MgO}$ and $\mathrm{Si} / \mathrm{SiO}_{2}$ samples.

\begin{tabular}{|c|c|c|c|c|c|}
\hline Substrate & & & $R_{P}(\Omega)$ & $R_{\mathrm{AP}}(\Omega)$ & TMR \\
\hline $\mathrm{Si}$ & & & 864 & 1583 & $83 \%$ \\
\hline $\mathrm{MgO}$ & & & 1411 & 2400 & $70 \%$ \\
\hline Substrate & $V_{\mathrm{P}}(\mu \mathrm{V})$ & $V_{\mathrm{AP}}(\mu \mathrm{V})$ & $S_{\mathrm{P}}\left(\frac{\mu \mathrm{V}}{\mathrm{K}}\right)^{\mathrm{a}}$ & $S_{\mathrm{AP}}\left(\frac{\mu \mathrm{V}}{\mathrm{K}}\right)^{\mathrm{a}}$ & TMS \\
\hline $\mathrm{Si}$ & 1.34 & 1.39 & -223 & -232 & $3.7 \%$ \\
\hline $\mathrm{MgO}$ & 3.00 & 3.08 & -750 & -770 & $2.6 \%$ \\
\hline
\end{tabular}

\subsubsection{Thermocurrent measurements}

In an open circuit, the Seebeck effect creates a voltage in an MTJ experiencing a temperature gradient, whereas in a closed circuit geometry, it can drive a Seebeck current. Fig. 3.3 shows the magnetization dependence of the Seebeck voltage and Seebeck current induced by laser heating with a power of $150 \mathrm{~mW}$ for an MTJ on $\mathrm{MgO}$ with an area of $1.57 \mu \mathrm{m}^{2}$ and a resistance of $28.1 \mathrm{k} \Omega$ in the antiparallel and $16.7 \mathrm{k} \Omega$ in the parallel states. Note that the laser power is considerably larger than in the first example (Fig. 3.2). The voltage varies between $93.30 \mu \mathrm{V}$ in the antiparallel and $90.72 \mu \mathrm{V}$ in the parallel state, whereas the current behaves inversely such that it reaches $4.90 \mathrm{nA}$ and $6.07 \mathrm{nA}$, respectively. This yields a TMS ratio of $2.84 \%$ and a current effect-ratio of $23.9 \%$.

Since the moments in Eq. (3.4), which depend on the magnetization dependent transmission $T(E)$, occur in the conductance $G$ as well as in the Seebeck coefficient $S$ (Eq. (3.3)), both, the voltage and the current should exhibit a magnetic field dependent variation, as suggested by Eq. (3.2). This prediction is confirmed by our experimental results. The difference in the effect amplitudes is explained by the fact that, as it can be seen in Eq. (3.2), the voltage only depends on the Seebeck coefficient $S$, whereas the current is additionally dependent on the electrical 


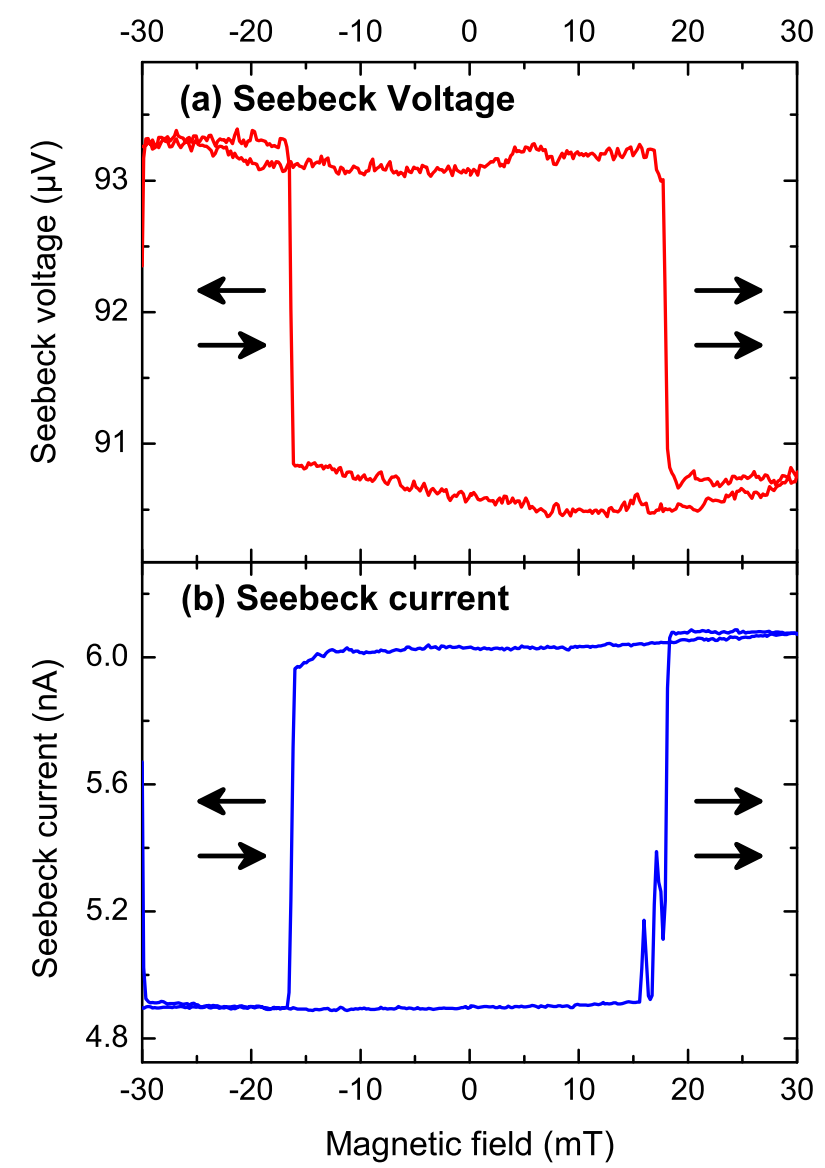

Figure 3.3: High laser power: Seebeck voltage (a) and Seebeck current (b) measured at a laser power of $150 \mathrm{~mW}$.

conductance $G$, which is strongly dependent on the magnetization alignment due to the high TMR ratio.

Seebeck currents were also investigated by Lin et al. ${ }^{37}$ but in contrast to our measurements they do not detect a dependency of the current on the magnetic field which they explain by the different mechanisms causing TMS in alumina-based MTJs. On Co-Fe-B/MgO MTJs, Liebing et al. demonstrated magnetic switching in Seebeck current measurements very recently. ${ }^{41}$

\subsubsection{Time-dependent thermovoltage signals}

To gain a deeper understanding of the processes leading to the TMS signal measured by the lock-in amplifier, a closer investigation of the time-dependent voltage signal is essential. It is assumed that the temperature gradient rapidly increases and decreases upon laser on/off, which is justified by temperature simulations yielding a time of $<2 \mu$ s to reach equilibrium. Thus, a nearly rectangular time-dependent voltage signal is expected corresponding to the laser modulation.

In Fig. 3.4, the time-dependent voltage signals of MTJs on $\mathrm{Si} / \mathrm{SiO}_{2}$ and $\mathrm{MgO}$ are 
Figure 3.4: Time-dependent voltage signals of MTJs on (a) $\mathrm{Si} / \mathrm{SiO}_{2}$ and (b) $\mathrm{MgO}$ substrate with a laser power of $10 \mathrm{~mW}$ and $15 \mathrm{~mW}$, respectively. As blue circles Simulation Program with Integrated Circuit Emphasis (SPICE) simulations are shown, as described in Sec. 3.5.

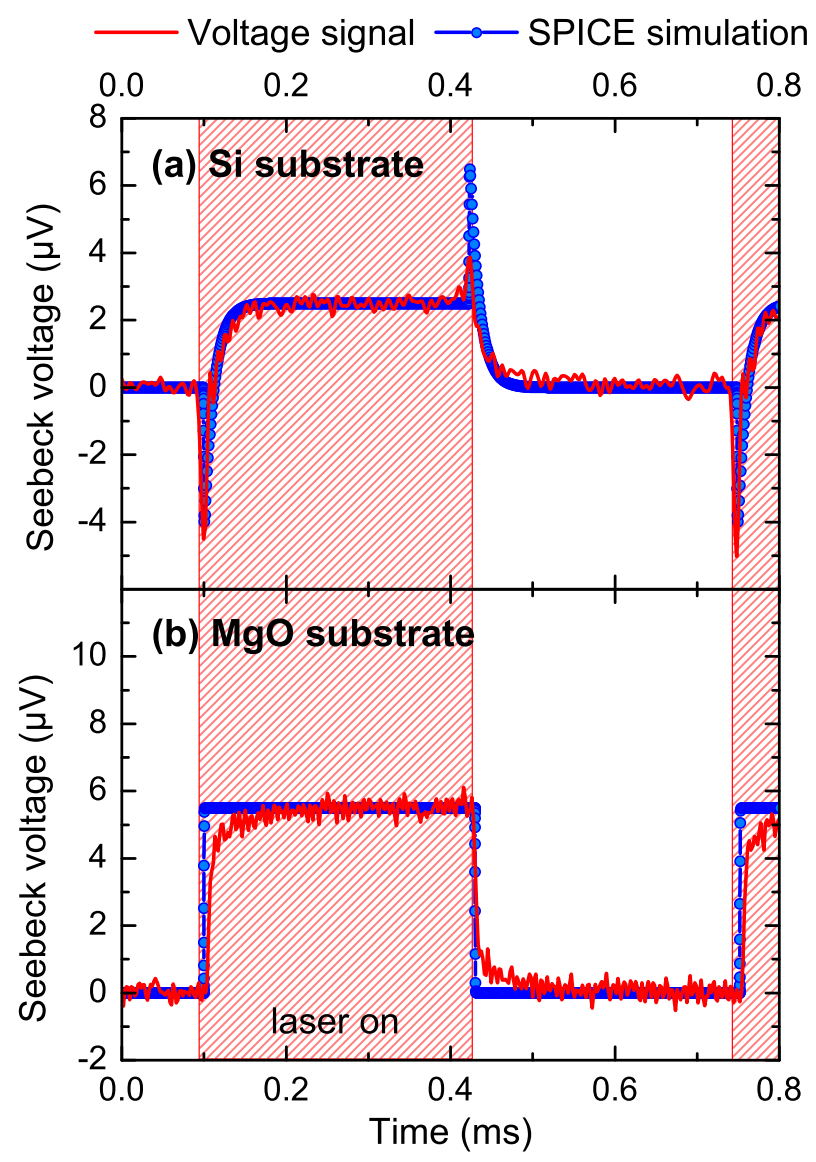

depicted. The traces for both substrate materials clearly reveal voltage plateaus with a small rise and fall-time when the laser is turned on and off. Whereas on $\mathrm{MgO}$ the rectangular shape is clearly visible, unexpected negative and positive voltage peaks can be additionally observed at the start and end of the heating period on $\mathrm{Si} / \mathrm{SiO}_{2}$. The position and shape of these voltage peaks suggest an electrical capacitance as their origin. The source can be further restricted to the substrate as the additional voltage only occurs in samples with p-doped Si substrate which is capacitively coupled to the bottom electrode by the $50 \mathrm{~nm} \mathrm{SiO}_{2}$ dielectric.

\subsection{Development of a model circuit}

Uncovering the processes responsible for the strikingly different temporal voltage traces measured on $\mathrm{MgO}$ and $\mathrm{Si} / \mathrm{SiO}_{2}$ substrates can be achieved by describing the sample structure as a model circuit. Fig. 3.5 sketches how the relevant parts inside the sample can be converted into an equivalent circuit consisting of three major units.

The first part is the MTJ simplified as a voltage source $V_{\text {MTJ }}$ simulating the 

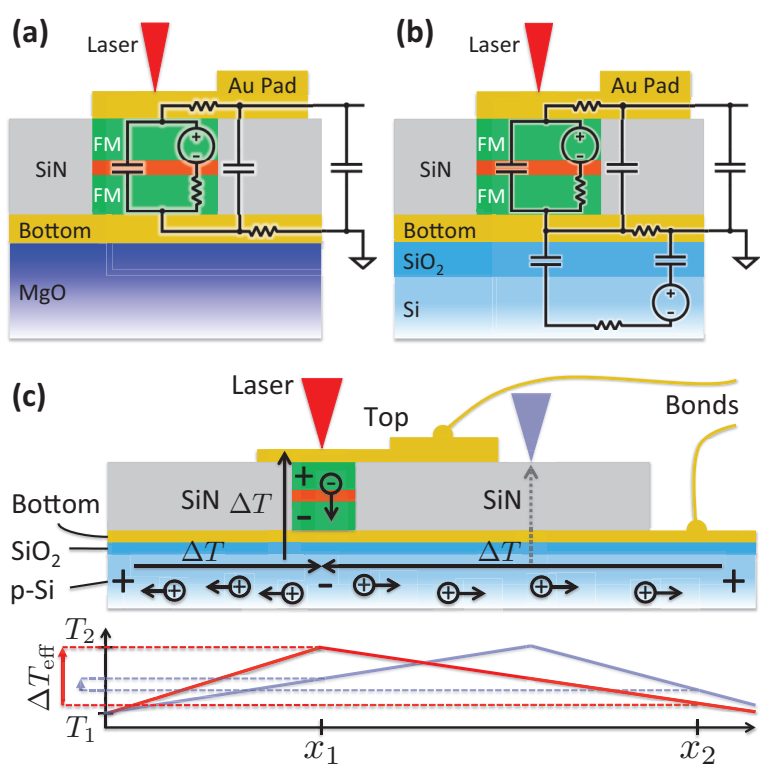

Figure 3.5: Model circuit for MTJs on (a) insulating $\mathrm{MgO}$ and (b) capacitively coupled p-type $\mathrm{Si}$ substrates. (c) Inside the samples on $\mathrm{Si}$ two heat gradients $\Delta T_{\mathrm{MTJ}}$ and $\Delta T_{\mathrm{Si}}$ produce thermovoltages $V_{\mathrm{MTJ}}$ and $V_{\mathrm{Si}}$, respectively. When the laser is positioned on the MTJ (red) the effective temperature gradient $\Delta T_{\text {eff }}$ between the contact points $x_{1}$ and $x_{2}$ is larger compared to the laser positioned between the MTJ and the edge of the sample (blue).

Seebeck voltage generated by the temperature gradient across the barrier, a resistor $R_{\mathrm{MTJ}}$ representing the dielectric barrier and a capacitor $C_{\mathrm{MTJ}}$ describing the capacitance built up by the two ferromagnetic layers (FM) separated by the $\mathrm{MgO}$.

The second unit contains the electrodes and wiring including the resistance of the bottom electrode $R_{\text {bottom }}$ and of the top contact, mainly the gold bond pad $R_{\text {Au-pad }}$. Furthermore, a capacitance $C_{\text {Au-pad }}$ is build up by the gold pad and the bottom electrode separated by the insulator SiN surrounding the MTJs. It is supplemented by the cable capacitance $C_{\text {cable }}$ of the coaxial cables connecting the sample to the electronic equipment.

The third major component is the substrate. In case of $\mathrm{MgO}$ samples, the substrate is insulating and therefore has not to be taken into account when constructing a model circuit (Fig. 3.5(a)). When Si samples are depicted, the substrate is a ptype semiconductor that creates a Seebeck voltage $V_{\mathrm{Si}}$ when heated (Fig. 3.5(b)) that capacitively couples to the bottom electrode through the $\mathrm{SiO}_{2}$ capping. The temperature gradient inside the substrate arises when the MTJ is heated. Not only the upper side of the MTJ is heated when irradiated by the laser, but also the lower part near the substrate experiences an elevated temperature due to heat conduction through the layer stack. Thus, the temperature inside the substrate underneath the MTJ is higher than at the edges of the sample. The resulting temperature gradient creates a Seebeck voltage in the p-Si substrate as sketched in Fig. 3.5(c). This effect is included in the model circuit by adding a voltage source and two capacitances $C_{\mathrm{SiO}_{2}}$, one underneath the MTJ and another at the point where the bottom electrode is connected to the gold bond wire, as these two points confine the segment where a capacitively coupled voltage can be detected (Fig. 3.5(b)). 
The size of all capacitances can be calculated from the model of a parallel plate capacitor except the cable capacitance that is given by the manufacturer. The sum of the resistors $R_{\mathrm{MTJ}}, R_{\mathrm{bottom}}$ and $R_{\mathrm{Au} \text {-pad }}$ connected in series has to match the measured TMR whilst the value of $R_{\text {bottom }}$ and $R_{\mathrm{Si}}$ can be deduced from the geometric dimensions of the conduction channel constituting between the MTJ and the contact point of the bottom electrode to the gold bond wire.

Fig. 3.4 shows a good agreement of SPICE simulations of the model circuit with the measured data. Our model of a Seebeck voltage created in the silicon substrate that cannot occur inside the $\mathrm{MgO}$ substrate explains the effects observed in the experiment. The absolute values of the voltages $V_{\mathrm{MTJ}}$ and $V_{\mathrm{Si}}$ have to be deduced. All other components are calculated and summarized in Table 3.3. Considering that the relation of the amplitude $A$ detected by the oscilloscope and the voltage output $V$ of the lock-in amplifier is given by $V \approx 0.5 \cdot A$ for a square wave signal, the measurements of Fig. 3.4 agree very well with those of Fig. 3.2. Based on these results, we are able to assign the different measured voltages to a Seebeck effect

Table 3.3: Basic estimations for calculating the resistance and capacitance in the model circuit for simulations.

\begin{tabular}{|c|c|c|}
\hline Comp. & How to calculate & Value \\
\hline$R_{\mathrm{MTJ}}$ & Extracted from TMR data & \\
\hline$V_{\mathrm{MTJ}}$ & Fit to data (value of plateau) & \\
\hline$V_{\mathrm{Si}}$ & Fit to data & \\
\hline$C_{\mathrm{MTJ}}$ & $\begin{array}{l}\text { Parallel plate capacitor: } \mathrm{MgO} \text { thickness, MTJ } \\
\text { area }\end{array}$ & $1.08 \mathrm{pF}$ \\
\hline$R_{\text {Au-pad }}$ & $\begin{array}{l}\text { Geometric dimensions of } \mathrm{Au} \text { pad and resistivity of } \\
\mathrm{Au}\end{array}$ & $10 \Omega$ \\
\hline$C_{\text {Au-pad }}$ & $\begin{array}{l}\text { Parallel plate capacitor: SiN thickness, area of } \\
\text { bond pad }\end{array}$ & $2.8 \mathrm{pF}$ \\
\hline$C_{\text {cables }}$ & Given by manufacturer & $160 \mathrm{pF}$ \\
\hline$C_{\mathrm{SiO}_{2}}$ & $\begin{array}{l}\text { Effective parallel plate capacitor with dielectric } \\
\mathrm{SiO}_{2}\end{array}$ & $70 \mathrm{nF}$ \\
\hline$R_{\mathrm{Si}}$ & $\begin{array}{l}\text { Geometric dimension of Si substrate, conducting } \\
\text { channel is created between MTJ and bond wire } \\
\text { contact to bottom electrode }\end{array}$ & $300 \Omega$ \\
\hline$R_{\text {bottom }}$ & $\begin{array}{l}\text { Geometric dimensions of conducting channel in- } \\
\text { side the bottom electrode between MTJ an bond } \\
\text { wire contact }\end{array}$ & $40 \Omega$ \\
\hline
\end{tabular}


inside the MTJ on the one hand and a Seebeck effect inside the substrate material on the other hand.

\subsection{Position-dependent measurements}

The tunnel magneto-Seebeck effect should arise from Seebeck voltages generated by a temperature gradient across the $\mathrm{MgO}$ layer sandwiched between two ferromagnets. From the underlying geometry of the MTJ sketched in Fig. 3.5(c) we conclude that moving the laser spot away from the junction on the gold bond pad should already decrease this temperature gradient and hence the observed Seebeck voltage. Furthermore, lateral temperature gradients created by the laser spot on the gold bond pad should cancel out because of the lateral radial symmetry of the heating.

We performed several position-dependent measurements in the vicinity of the gold bond pad of the junction, and also with the laser spot moved more than $1 \mathrm{~mm}$ away from the MTJ. The inset of Fig. 3.6(a) shows the geometry of the bond pad with the MTJ located at position P1. The area heated by the laser is sketched as a red circle. Its diameter is according to simulations ${ }^{57}$ only slightly larger than the laser spot $(d=10 \mu \mathrm{m})$. TMS measurements were taken with the laser positioned at P1 - P4. The corresponding time-dependent voltage signals are shown in Fig. 3.6(a). It can be seen that a square-wave-like Seebeck voltage that is attributed to the MTJ, as discussed in Secs. 3.4-3.5, occurs only if the laser spot is centered directly onto the MTJ at P1. Only at this position the TMS effect is observed. At position P2, adjacent to the MTJ, the Seebeck voltage of the MTJ is already strongly reduced. Only the voltage peaks attributed to voltages generated in the Si substrate occur at all four positions. Time-dependent voltage signals were recorded also for large distances, which are shown in Fig. 3.6(b): It is observed that the time constant of the exponential decay of the voltage peaks increases with distance. The plotted simulated curves describe the signals reasonably well. In the model, the MTJ voltage $V_{\mathrm{MTJ}}$ is set to zero for non-zero distances between the laser and the MTJ and the voltage peaks can be reproduced by adjusting the resistance of the substrate and by lowering the generated voltage (reduced effective temperature gradient, Fig. 3.5(c)) according to the increased distance.

These findings support the attribution of the voltage peaks to parasitic voltages of the $\mathrm{Si} / \mathrm{SiO}_{2}$ substrate. Further, they show that the setup enables us to discriminate voltages locally generated in a single MTJ.

\subsection{Conclusion}

In summary, we have presented an experimental setup that allows the reliable measurement of thermomagnetoelectric effects in a single tunnel junction with nanovolt resolution at a temporal resolution of a few microseconds. MTJs grown on oxidized 
(a)

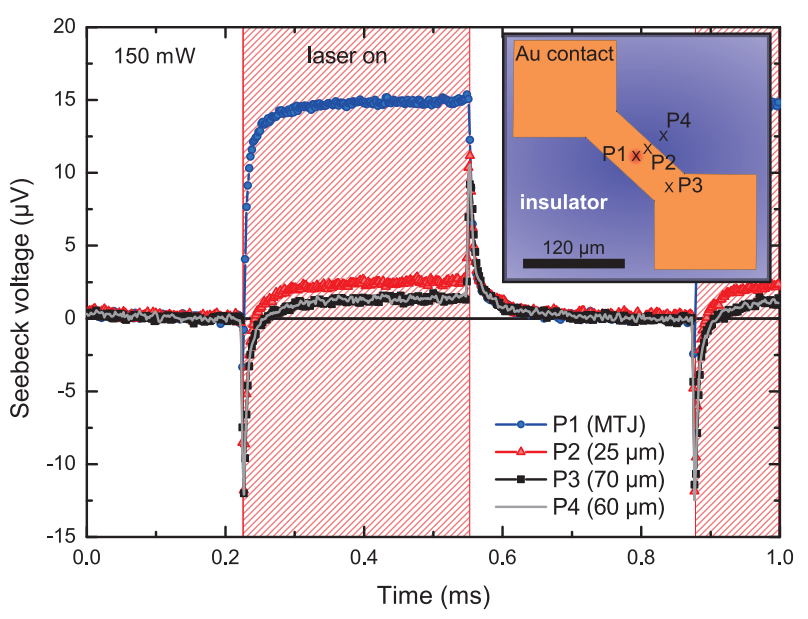

(b)

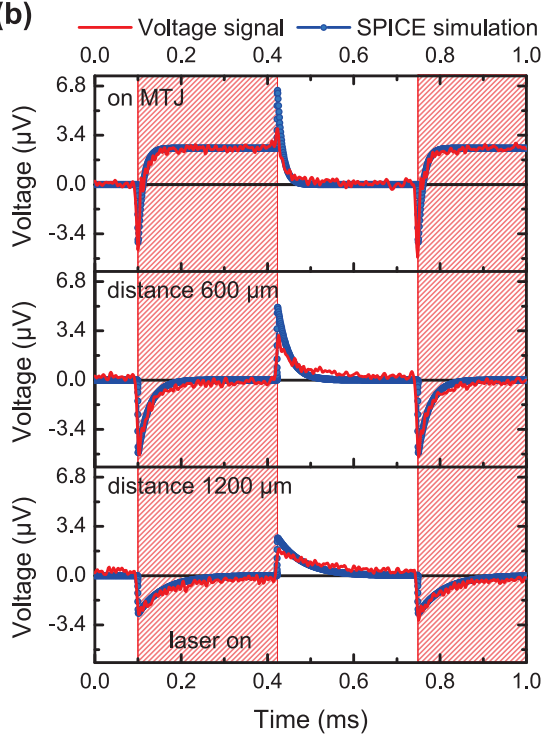

Figure 3.6: (a) Short distances: Measurements in the vicinity of the MTJ; the positions are shown in the inset. Laser spot and MTJ are located at P1, the heated area is sketched in red. (b) Large distances: Measurements of a different MTJ at distances more than factor of 10 larger as in the case of (a).

$\mathrm{Si}$ and $\mathrm{MgO}$ substrates have been tested and show comparable Seebeck voltages and currents. We find a magnetic effect also in the Seebeck current measurements. Further, with the improved temporal resolution, the voltage signals of the MTJs can be interpreted with the help of a model circuit. On oxidized Si substrates an additional voltage generated in the substrate can be identified. However, no evidence is found that this voltage influences the TMS measurements carried out with a lock-in amplifier. Distance dependent measurements reveal that the detected Seebeck voltage originates only from the MTJ layer stack. When the laser is moved away from the MTJ, only the Seebeck voltage signal of the substrate can be found.

\subsection{Acknowledgments}

M.M., A.T., and G.R. are supported by the Deutsche Forschungsgemeinschaft (DFG) through SPP 1538 SpinCaT (MU1780/8-1, RE1052/24-1). V.D. and A.T. are supported by the Ministry of Innovation, Science and Research (MIWF) of North Rhine-Westphalia with an independent researcher grant. 


\title{
Chapter 4
}

\section{Parameter space for thermal spin transfer torque}

\author{
J.C. Leutenantsmeyer, M. Walter, V. Zbarsky, et al. \\ SPIN 3, 1350002 (2013) \\ DOI: $10.1142 / S 2010324713500021$
}

\begin{abstract}
Thermal spin-transfer torque describes the manipulation of the magnetization by the application of a heat flow. The effect has been calculated theoretically by Jia et al. in 2011. It is found to require large temperature gradients in the order of Kelvins across an ultra thin $\mathrm{MgO}$ barrier. In this paper, we present results on the fabrication and the characterization of magnetic tunnel junctions with three monolayer thin $\mathrm{MgO}$ barriers. The quality of the interfaces at different growth conditions is studied quantitatively via high-resolution transmission electron microscopy imaging. We demonstrate tunneling magnetoresistance ratios of up to $55 \%$ to $64 \%$ for 3 to 4 monolayer barrier thickness. Magnetic tunnel junctions with perpendicular magnetization anisotropy show spin-transfer torque switching with a critical current of $0.2 \mathrm{MA} / \mathrm{cm}^{2}$. The thermally generated torque is calculated ab initio using the Korringa-Kohn-Rostoker and nonequilibrium Green's function method. Temperature gradients generated from femtosecond laser pulses were simulated using COMSOL, revealing gradients of $20 \mathrm{~K}$ enabling thermal spin-transfer-torque switching.
\end{abstract}

\subsection{Introduction}

Spintronic devices provide excellent opportunities for data storage applications. Today, magnetic random access memory (MRAM) has shown several advantages to conventional RAM. Besides faster access times also higher storage density, lower power consumption and nonvolatility are obtained. ${ }^{16}$

Spincaloric effects in magnetic tunnel junctions (MTJs) may provide a great way of using excess heat for storage devices. ${ }^{11,35}$ Utilizing effects such as the tunneling magneto-Seebeck (TMS) effect, the energy efficiency of memory will be enhanced and mark a next step towards a greener information technology. This effect was 
recently predicted theoretically ${ }^{36}$ and observed experimentally. ${ }^{37,40,49}$

In addition to the TMS effect, thermal spin-transfer torque (T-STT) has been proposed by Slonczewski ${ }^{43}$ in 2010 and calculated by Jia et al. ${ }^{44}$ in 2011 . Corresponding to the conventional spin-transfer torque effect ${ }^{59,60}$, a spin-polarized tunneling current is used to switch the state of an MTJ. In case of T-STT, this current is generated from the thermally excited electron transport across the tunneling barrier.

The theoretical considerations of T-STT lead to experimental challenges for the sample preparation and the experiments: 3 monolayer (ML, $0.63 \mathrm{~nm}$ ) thin $\mathrm{MgO}$ barriers as well as large temperature gradients in the order of Kelvins across this barrier are required. ${ }^{44}$ Here, we demonstrate that all requirements can be fulfilled.

\subsection{Sample Preparation}

The samples are prepared on thermally oxidized silicon substrates in an ultra high vacuum (UHV) chamber. The MTJ stack consists of Ta $(10 \mathrm{~nm}) / \mathrm{Co}-\mathrm{Fe}-\mathrm{B}(2.5)$ / $\mathrm{MgO}(0.63-2.1)$ / Co-Fe-B (5.4) / Ta (5.0) / Ru (3.0). The thickness of the MgO is varied from $3 \mathrm{ML}(0.63 \mathrm{~nm})$ up to $10 \mathrm{ML}(2.1 \mathrm{~nm})$. For MTJs with perpendicular magnetization anisotropy (PMA) the thickness of the Co-Fe-B electrodes is reduced to 1.0 and $1.2 \mathrm{~nm}$.

Tantalum and $\mathrm{Co}-\mathrm{Fe}-\mathrm{B}$ are deposited in a magnetron sputter chamber, while the $\mathrm{MgO}$ barrier and the ruthenium capping layer are e-beam evaporated in an adjacent chamber without breaking the vacuum. The layer stack is annealed ex situ to crystallize the Co-Fe-B electrodes in a solid-state epitaxy process.

After annealing, the samples are patterned using standard UV- and electronbeam lithography and structured by argon ion milling down to diameters of $150 \mathrm{~nm}$.

\subsection{Growth of Ultra Thin Barriers}

Growing ultra thin $\mathrm{MgO}$ barriers introduces experimental challenges, because crystalline $\mathrm{MgO}$ barriers give rise to large TMR ratios due to their spin-filter effect. ${ }^{61,62}$ This effect is decreased for thin barriers. ${ }^{38}$ While thick barriers of $10 \mathrm{ML}$ thickness can yield TMR ratios of over $600 \%$ at room temperature, this value is strongly decreased for thin tunnel barriers. ${ }^{15}$ A decrease of the spin polarization from additional d-like contributions was reported for alumina junctions as well. ${ }^{63}$

$\mathrm{MgO}$ deposited on amorphous Co-Fe-B usually crystallizes in the (001) direction if five or more ML are deposited. The Co-Fe-B crystallizes during annealing, while $\mathrm{MgO}$ acts as a template for the solid-state epitaxy process ${ }^{64}$ and long range order on the atomic scale is induced at $\mathrm{Co}-\mathrm{Fe}-\mathrm{B} / \mathrm{MgO}$ interfaces. ${ }^{65}$ For ultra thin barriers, this effect is not present and thus the interface quality is reduced. A successful 

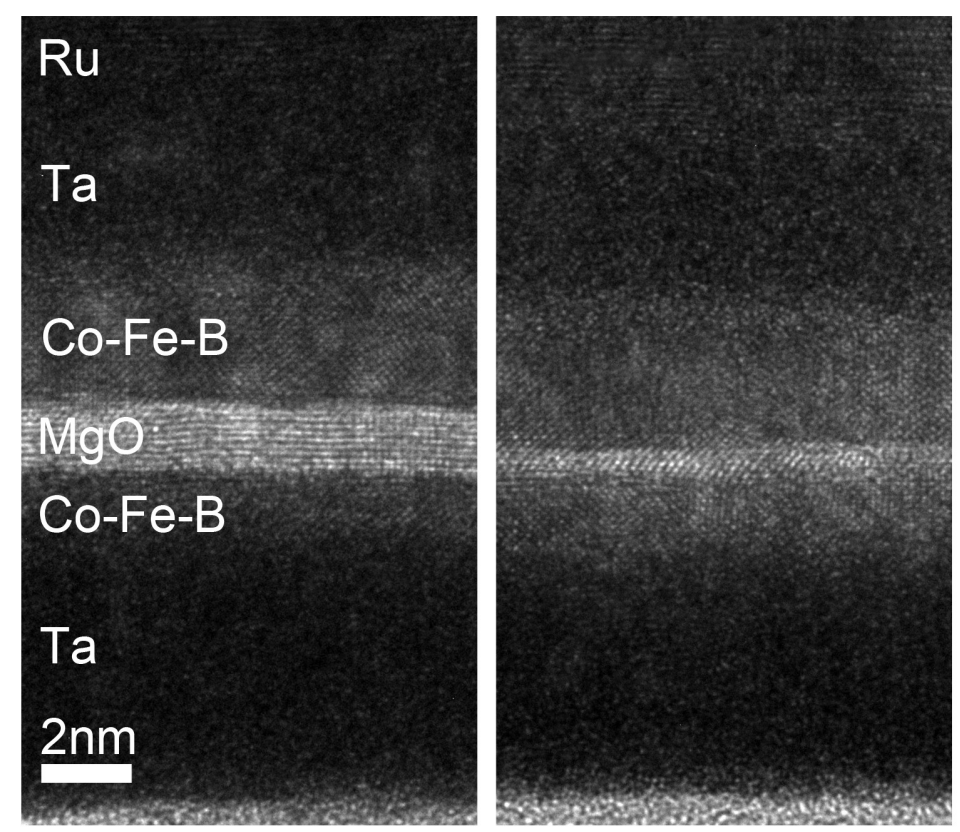

Figure 4.1: HRTEM images of a thick $10 \mathrm{ML}$ (left) and a heated $3 \mathrm{ML} \mathrm{MgO}$ barrier (right). The IQR values are $(5.6 \pm 1.5)^{\circ}(10 \mathrm{ML})$ and $(6.7 \pm 0.8)^{\circ}(3 \mathrm{ML})$.

preparation of a TMR device below $5 \mathrm{ML}$ of $\mathrm{MgO}$ has to focus on a crystalline growth below that critical thickness.

The effect of the deposition rate and influence of substrate temperature on the shift of the critical $\mathrm{MgO}$ crystallization thickness has been discussed by Kurt et al. and Isogami et al. A threshold value of $5 \mathrm{pm} / \mathrm{s} \mathrm{MgO}$ deposition rate is reported to obtain high quality tunnel barriers. Also, infrared heating to $300^{\circ} \mathrm{C}$ is reported to enhance the TMR ratio of $4 \mathrm{ML}$ thick barriers reaching values of more than $200 \% .{ }^{66,67}$ The interfaces of our junctions exhibit their best properties at deposition rates of $1 \mathrm{pm} / \mathrm{s}$. Furthermore, we investigated the effect of sample annealing between $100^{\circ} \mathrm{C}$ and $350^{\circ} \mathrm{C}$ during e-beam evaporation of the $\mathrm{MgO}$ barrier.

\subsection{Quantitative HRTEM Analysis of MgO Barriers}

Transmission electron microscopy (TEM) was conducted at a Philips CM200 FEG UT under $200 \mathrm{kV}$, with cross-sectional specimens prepared by a FEI Nova NanoLab 600 Focused Ion Beam under $30 \mathrm{kV}$ with a final polishing step at $5 \mathrm{kV}$. High resolution TEM images of the $\mathrm{MgO}$ barrier were Fourier-filtered around the $\mathrm{MgO}$ growth direction and $\mathrm{MgO}$ reciprocal lattice constant, which results in the $\mathrm{MgO}$ layer being represented by distorted lines arising from its crystal texture. This effect is used 
to estimate the degree of texturing of the layer by transforming the image into a two-dimensional line parameter space using the Hough transform. ${ }^{68}$ The texture leads to intensity peaks with finite distributions in the transformed image. The width of these distributions is estimated by their inter-quartile range (IQR) for the parameter pairs of several lines, whose average value is used as the approximation for one image.

For example, the high degree of texturing of the $10 \mathrm{ML}$ layer in Fig. 4.1 (left) is estimated as $(5.6 \pm 1.5)^{\circ}$, whereas the lower degree of texturing of the $3 \mathrm{ML}$ layer in Fig. 4.1 (right) gives a value of $(6.7 \pm 0.8)^{\circ}$.

This allows us to quantify the degree of order and to optimize the barrier growth conditions. We applied the method to find the optimal $\mathrm{MgO}$ growth temperature. The IQR value is strongly decreased with an $\mathrm{MgO}$ growth temperature of $100^{\circ} \mathrm{C}$. However, we found no further enhancement for growth temperatures higher than $100^{\circ} \mathrm{C}$ (Fig. 4.2).

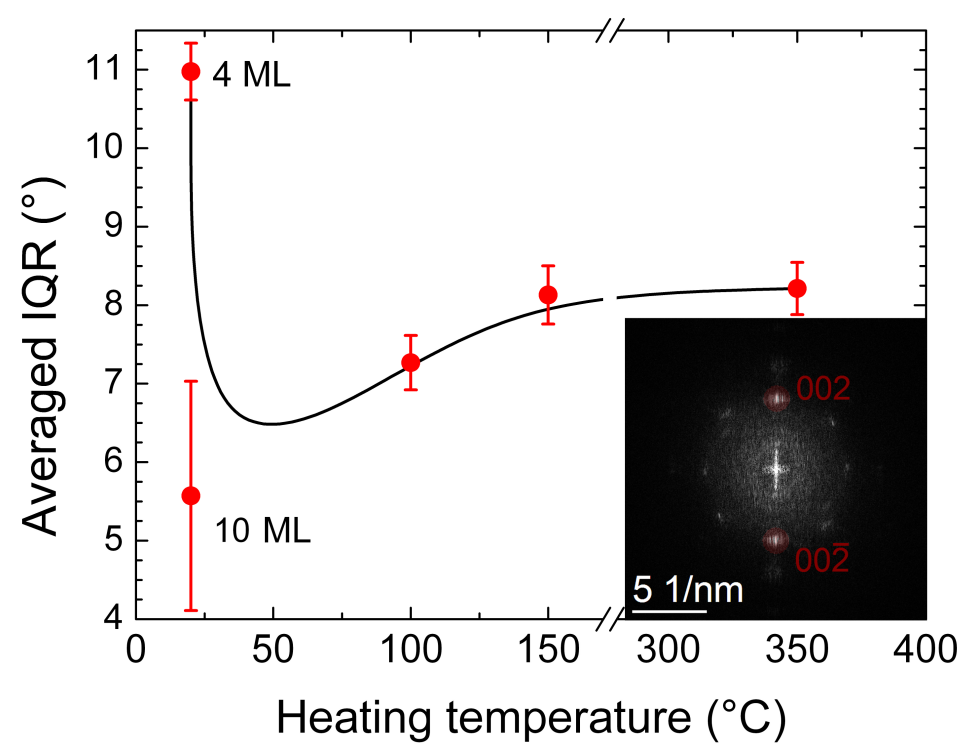

Figure 4.2: Quantitative analysis of the HR-TEM images of ultra thin MgO barriers at different growth temperatures. The black line is a guide to the eye. The IQR values for room temperature and $100^{\circ} \mathrm{C}$ were obtained in $4 \mathrm{ML}$ MTJs, $150^{\circ} \mathrm{C}$ and $350^{\circ} \mathrm{C}$ in $3 \mathrm{ML}$ MTJs. The inset shows the space frequencies (red) used for Fourier filtering of the $3 \mathrm{ML} \mathrm{MgO}$ barrier from Fig. 4.1. 


\subsection{Spin-transfer Torque in PMA Junctions}

First, $\mathrm{R}(\mathrm{H})$ and $\mathrm{I}(\mathrm{U})$ measurements are carried out to characterize the samples. The 10 ML MgO barriers show a TMR ratio of up to $270 \%$ and a large TMS effect of up to $50 \%$. For $3 \mathrm{ML}$ of $\mathrm{MgO}$, a TMR ratio of up to $55 \%$ is found.

New materials with high perpendicular magnetization anisotropy are important for high density storage applications. For example, MTJs with Mn-Ga electrodes are very promising due to their high PMA. ${ }^{69}$ Fortunately, PMA has been demonstrated for Co-Fe-B films below a critical thickness. Due to the small thickness, which is approximately equal to the absorption length of the transferred torque, this results in a reduction of the critical switching current $J_{c} \cdot{ }^{70,71}$ Secondly, $J_{c}$ is reduced, because the increase in PMA in these junctions lowers the influence of the out-of-plane demagnetizing field which is one of the factors responsible for a high $J_{c} \cdot{ }^{72,73}$ As a consequence, we expect that this reduction of $J_{c}$ also applies to the thermally driven electron transport through the device and thus enhances the

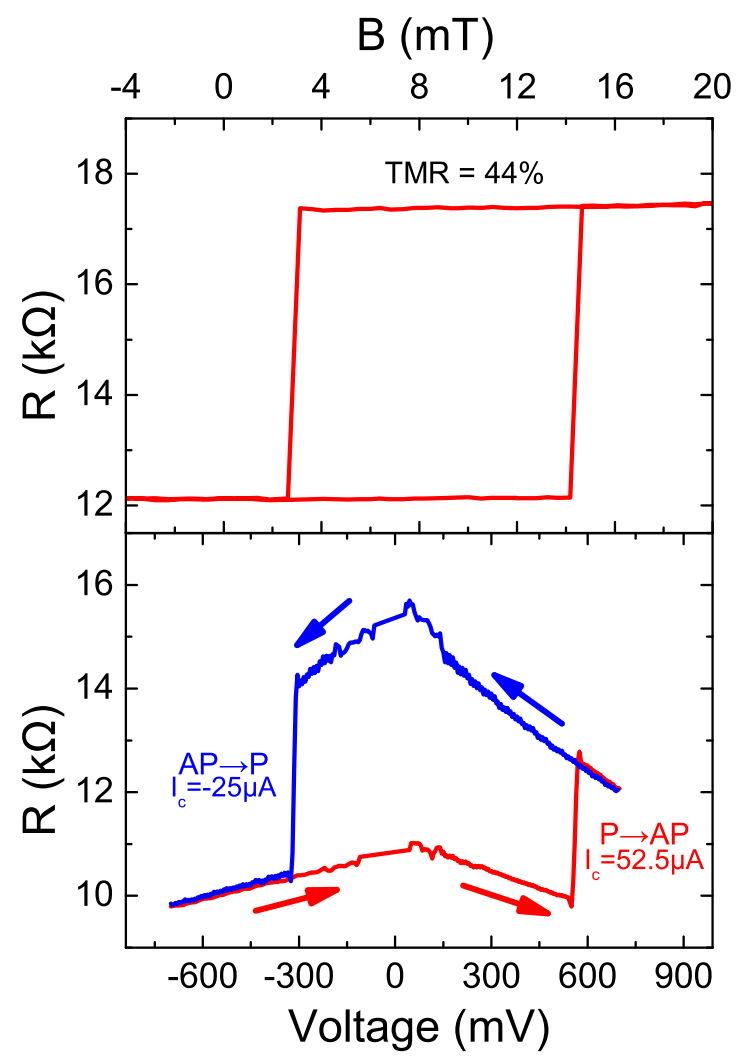

Figure 4.3: Electrical characterization of an MTJ with 4ML MgO barrier and PMA. top: minor loop, bottom: RV-characteristic with an applied field of $8.6 \mathrm{mT}$. 
possibility of observing T-STT.

Thus, the thickness of both Co-Fe-B layers was reduced to $1.0 \mathrm{~nm}$ and $1.2 \mathrm{~nm}$. The resistance is plotted as function of the magnetic field perpendicular to the plane in Fig. 4.3. The upper viewgraph shows PMA for this thickness range of both magnetic layers. The $\mathrm{MgO}$ thickness is $4 \mathrm{ML}(0.84 \mathrm{~nm})$ in this case with a maximum TMR ratio of $64 \%$. For the TMS measurements the MTJ was heated from the top with a diode laser (wavelength $640 \mathrm{~nm}$, power up to $150 \mathrm{~mW}$ ), modulated by a frequency generator at $1.5 \mathrm{kHz}$. The voltage is then recorded using a lock-in amplifier (see Ref. [49] for more details). A TMS effect of $6 \%$ was observed.

The lower graph of Fig. 4.3 shows the junction resistance as a function of the applied bias voltage with a magnetic offset field of $8.6 \mathrm{mT}$. Spin-transfer torque switching from the antiparallel (AP) to the parallel (P) state as well as from the P to the AP state can be seen. The corresponding critical currents are $-25 \mu \mathrm{A}$ and $52.5 \mu \mathrm{A}$. Given a diameter of $155 \mathrm{~nm}$ of the circular junction and using an average critical current of $38.5 \mu \mathrm{A}$, the average critical current density equals $0.2 \mathrm{MA} / \mathrm{cm}^{2}$. This value is much lower than the critical current densities of more than $0.8 \mathrm{MA} / \mathrm{cm}^{2}$ reported by other groups so far. ${ }^{71,74,75}$

\subsection{Torque Simulations}

We also perform ab initio calculations based on density functional theory. In particular, we use the Korringa-Kohn-Rostoker and the nonequilibrium Green's function method $^{46,70}$ to obtain the thermal spin-transfer torque of the tunnel junction. In particular, the torque acting on the atomic layer $i$ is given by

$$
\vec{\tau}_{i}(E)=\frac{1}{\hbar} \Delta_{i} \hat{M}_{i} \times \delta \vec{m}_{i}(E)
$$

where $\Delta_{i}$ is the exchange energy, $\vec{M}_{i}$ is the magnetic moment, and $\hat{M}_{i}=\vec{M}_{i} / M_{i}$. The change in the magnetic moment in each layer $\delta \vec{m}_{i}$ due to the current is calculated using the nonequilibrium Green's function formalism where the details of our implementation are given in Ref. [70]. To get the energy-dependent torque acting on the free layer $\tau^{\text {free }}(E)$, we sum up over all atomic layers within the free layer. By integrating over energy we get for the total torque in the free layer

$$
\tau^{\text {free }}=\int\left(\tau_{L \rightarrow R}^{\text {free }} f_{L}\left(E, T_{L}, \mu\right)+\tau_{R \rightarrow L}^{\text {free }} f_{R}\left(E, T_{R}, \mu\right)\right) d E,
$$

where $f_{L(R)}$ and $T_{L(R)}$ are the occupation function and temperature of the left (right) lead. $\mu$ is the electro-chemical potential and $\tau_{L \rightarrow R(R \rightarrow L)}^{\text {free }}$ is the torque acting on the free layer originating from electrons going from left to right (right to left). The system consists of a tunnel barrier with $3 \mathrm{ML} \mathrm{MgO}$ between two ferromagnetic Fe leads with $20 \mathrm{ML}$ and $\mathrm{Cu}$ as a reservoir. 

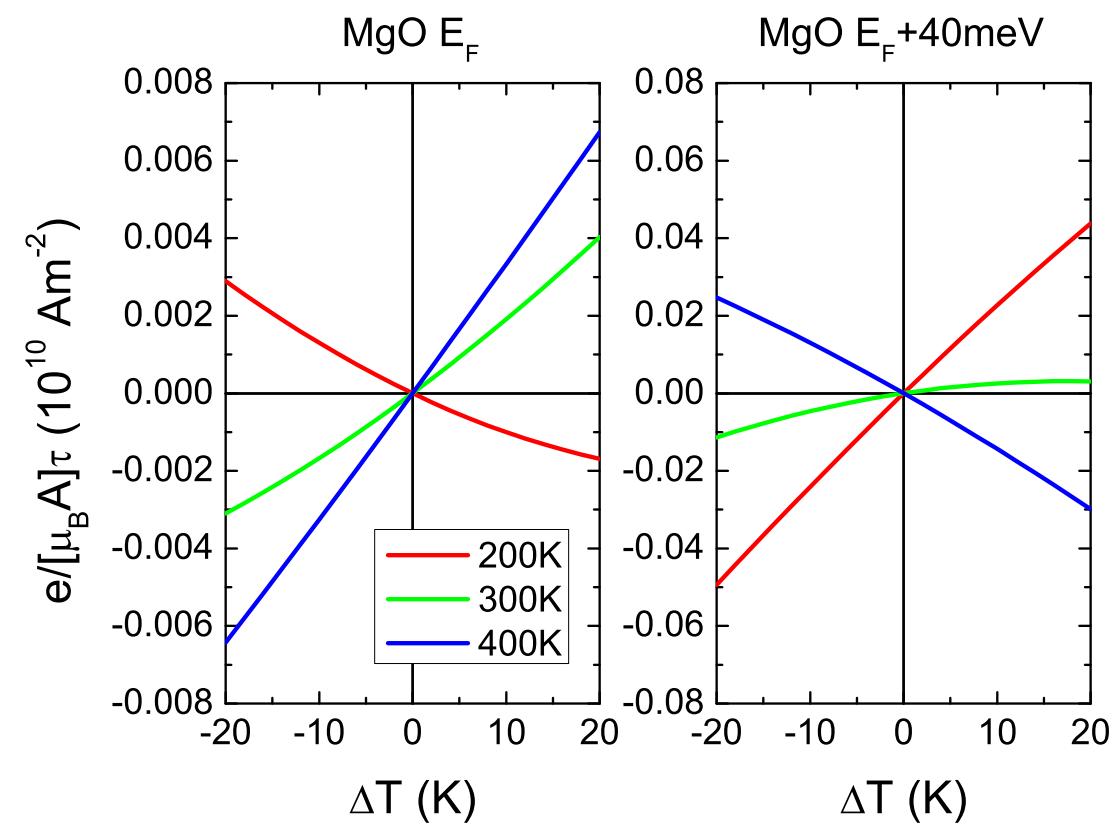

Figure 4.4: Calculated thermal torque at different lead temperatures for a $3 \mathrm{ML}$ $\mathrm{MgO}$ barrier (left). With shifting the CoFe Fermi level by $40 \mathrm{meV}$ (right) the acting torque can be enhanced significantly.

The resulting thermal spin-transfer torque for different temperatures of the left lead as a function of the temperature difference is shown in Fig. 4.4 (left). The slope of the function changes with different temperatures. However, the size of the torque is small in comparison to the bias driven spin-transfer torque. ${ }^{70}$ To simulate the influence of the Fe-Co alloy used as the electrodes, we adjust the Fermi level by a shift of $40 \mathrm{meV}$.

The resulting thermal spin-transfer torque is shown in Fig. 4.4 (right). Now, the size of the torque changes by one order of magnitude and is similar to the values obtained by Jia et al. ${ }^{44}$ Therefore, the position of the Fermi level, which can be adjusted by the composition of the Fe-Co alloy, is a key feature to maximize the thermal spin-transfer torque and has to be further investigated.

\subsection{Temperature Simulations}

It remains to show that a temperature gradient of larger than $10 \mathrm{~K}$ can be realized across our ultra thin barriers. Here, we want to utilize an ultrashort femtosecond laser pulse to generate large temperature gradients. Then, extreme conditions are attained by the very short deposition of the energy within a very thin layer at the top surface of the device. This allows temperature gradients of approximately $100 \mathrm{~K}$ within a few nanometer right after excitation and thermalization of the electrons. 
As the time evolves, this extreme temperature gradient will level out. We applied finite element simulations using the COMSOL Multiphysics numerical solver to estimate the temperature gradients across the $\mathrm{MgO}$ barrier that can be achieved in our experimental geometry.

The magnetic tunnel junction is simulated with a diameter of $200 \mathrm{~nm}$ and is modeled in a two dimensional, rotational symmetric geometry. The simulated layer stack is as follows: $3 \mu \mathrm{m}$ Si substrate/500 $\mathrm{nm} \mathrm{SiO}_{2} / 10 \mathrm{~nm} \mathrm{Ta} / 2.5 \mathrm{~nm}$ Co-Fe$\mathrm{B} / 0.63 \mathrm{~nm} \mathrm{MgO} / 5.4 \mathrm{~nm} \mathrm{Co}-\mathrm{Fe}-\mathrm{B} / 5 \mathrm{~nm} \mathrm{Ta} / 3 \mathrm{~nm} \mathrm{Ru} / 36.6 \mathrm{~nm} \mathrm{Au}$. According to our lithography process, the layers down to the $10 \mathrm{~nm}$ thick Ta layer are patterned as a junction pillar which is isolated by $50.4 \mathrm{~nm}$ of $\mathrm{SiO}_{2}$. The top strip line consists of $6.3 \mathrm{~nm} \mathrm{Cr}$ and $25.2 \mathrm{~nm} \mathrm{Au}$. The complete model has a diameter of $50 \mu \mathrm{m}$ which is large enough to cover the heating effect of the laser pulse with $w_{0} \approx 11 \mu \mathrm{m}$ (see below). COMSOL's heat transfer module solves the heat conduction equation in which the femtosecond laser pulse acts as heat source. In addition, we have to know the specific heat $c_{p}$, the density $\rho$ and the thermal conductivity $\kappa$ for the materials involved and these values can be found in Ref. [49]. The heat distribution provided by the laser pulse is modeled by the Lambert-Beer law and a scaling factor which includes the material's reflectance $R$ as well as the optical penetration depth $\lambda$. The spatial distribution of the pulse energy $E_{\text {pulse }}$ is assumed to be Gaussian with the beam waist $w_{0}$. The temporal shape of the laser pulse is Lorentzian with the
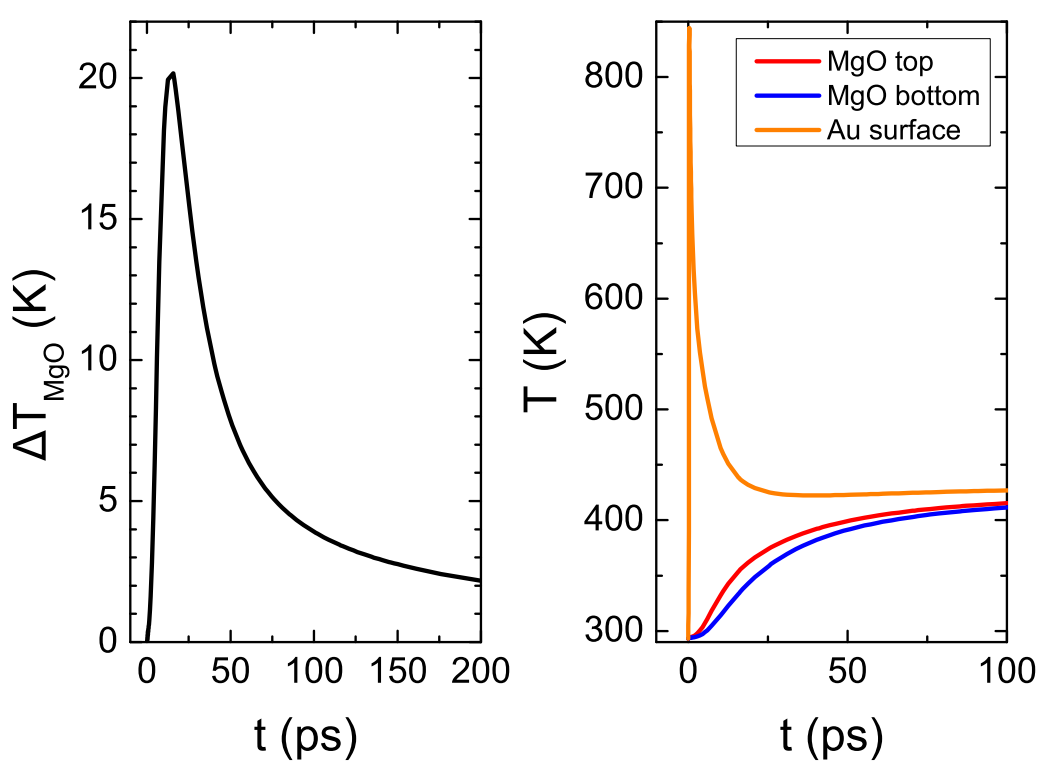

Figure 4.5: Left: Simulated temperature difference across a $0.63 \mathrm{~nm} \mathrm{MgO}$ tunnel barrier as a function of time. Right: Temperature of the heated gold surface and the two $\mathrm{MgO} / \mathrm{Co}-\mathrm{Fe}-\mathrm{B}$ interfaces. 
pulse duration $\Gamma$.

The parameter values are $E_{\text {pulse }}=0.2 \mu \mathrm{J}, w_{0}=11 \mu \mathrm{m}, \Gamma=50 \mathrm{fs}$, as measured in the confocal microscope setup used and described in Ref. [49]. The heat distribution provided by the laser pulse was calculated with the optical constants found in the literature for gold, ${ }^{76,77}$ namely a reflectivity at $800 \mathrm{~nm}$ of $R=0.975$ and an optical penetration depth at $800 \mathrm{~nm}$ of $\lambda=12.7 \mathrm{~nm}$. The thickness of the uppermost gold layer is almost twice its optical penetration depth. Therefore, most of the heat will be absorbed in the gold layer, which justifies taking only the optical constants for gold into account. The layers were discretized in a fine mesh with element sizes in the junction layers of $0.2-2 \mathrm{~nm}$.

In the right viewgraph of figure 4.5 the temperatures for the two $\mathrm{Co}-\mathrm{Fe}-\mathrm{B} / \mathrm{MgO}$ interfaces and the gold surface in the center of the junction are shown. The temperatures as well as their temporal evolution is reasonable for the two Co-Fe-B/MgO interfaces. As depicted in Fig. 4.5, the maximum temperature difference across the $\mathrm{MgO}$ interface is approximately $20 \mathrm{~K}$. Temperatures of more than $\Delta T=6.5 \mathrm{~K}$ are required for the T-STT switching from the antiparallel into the parallel state according to the calculations of Jia et $a l .{ }^{44}$ In total, we achieve a temperature difference of more than a few Kelvin for more than a hundred picoseconds for the pulse power and geometry simulated here.

\subsection{Conclusion}

We demonstrated the fabrication of magnetic tunnel junctions with ultra thin $\mathrm{MgO}$ barriers and small interface roughness. The samples of 3 ML to 4 ML barrier thickness showed high TMR ratios of $55 \%$ to $64 \%$ and spin-transfer torque switching with critical current densities as low as $0.2 \mathrm{MA} / \mathrm{cm}^{2}$. The texture of the junctions has been investigated by high-resolution TEM imaging quantitatively. We suggest the average inter-quartile range value of the $\mathrm{MgO}$ layer Hough transform as an quantitative indicator of the degree of texturing for the junction quality. This allows us to optimize the $\mathrm{MgO}$ barrier growth. The thermal torque has been calculated as a function of the temperature gradient. With adjusting the Fermi level via the Co-Fe-B composition, a maximum T-STT effect can be obtained. Temperature simulations of junctions heated by femtosecond laser pulses revealed large temperature gradients in the order of $10 \mathrm{~K}$ for around $100 \mathrm{ps}$. On the base of these parameters, we expect to observe T-STT in these kind of junctions. Thus, magnetic switching by applying only a temperature gradient will be feasible.

\subsection{Acknowledgments}

M.M. and M.S. are supported by German Research Foundation (DFG) through SFB 602. C.H., M.M. and A.T. are supported by the DFG through SPP 1538 
SpinCaT. A.T. is supported by the Ministry of Innovation, Science and Research (MIWF) of North Rhine-Westphalia with an independent researcher grant. G.R. is supported by the DFG through grant RE1052/22-1. 


\section{Chapter 5}

\section{Outlook}

This chapter deals with first results obtained in two additional series of measurements that were performed in the last months of this thesis. The discussion of chapter 2 is continued, on how the tunnel magneto-Seebeck effect in MTJs can be tuned. One possibility, presented in section 5.1, is to apply a DC bias voltage simultaneously to the laser heating. Another option is the change of material used as the MTJ's ferromagnetic electrode. In this regard, section 5.2 describes first results obtained from MTJs containing the Heusler compound $\mathrm{Co}_{2} \mathrm{FeSi}$ as ferromagnetic electrode. To check the applicability of MTJs as thermoelectric devices, the thermoelectric figure of merit is estimated in section 5.3.

\subsection{Bias voltage dependence of the tunnel magneto-Seebeck effect}

In chapter 2 , the requirements for a high tunnel magneto-Seebeck effect were discussed. The transmission probability of electrons through a magnetic tunnel junction at the Fermi level not only needs to be very different for parallel and antiparallel magnetization configuration of the two ferromagnetic electrodes, which leads to a high tunnel magnetoresistance ratio; it also requires a strong asymmetry of the transmission function with respect to the Fermi level, as sketched in Fig. 2.1 for the antiparallel transmission $T_{\mathrm{AP}}$, to achieve a high tunnel magneto-Seebeck effect.

The $\mathrm{Co}-\mathrm{Fe}-\mathrm{B} / \mathrm{MgO}$ system meets the above mentioned requirements on the spindependent electronic structure: As calculated by Butler et $a l .{ }^{38}$, as well as by Mathon and Umerski ${ }^{78}$, the spin-polarization of bcc-Fe and bcc-Co, which is caused by a fully spin-polarized $\Delta_{1}$ band, is enhanced by the complex band structure of $\mathrm{MgO}$. Electrons in not spin-polarized states that contribute to a spin-independent tunnel current decay much faster in the barrier than electrons in the $\Delta_{1}$ states. Experimentally, TMR ratios of up to $604 \%$ were observed in this system ${ }^{15}$.

The necessary asymmetry of the barrier's transmission function was studied in $a b$ initio calculations by Heiliger et $a l^{52}$. In that publication, it was predicted that the Seebeck coefficients and, hence, the tunnel magneto-Seebeck effect can be tailored by choice of $\mathrm{Co}-\mathrm{Fe}$ composition of the Co-Fe-B alloy. Fig. 5.1 shows the 
Figure 5.1: $A b$ initio calculations of TMS ratio and Seebeck coefficients. The composition of the $\mathrm{Co}-\mathrm{Fe}$ alloy is varied from pure Co to pure $\mathrm{Fe}$ in steps of $10 \%$ Fe content. a, TMS ratio of the alloys at room temperature for a $\mathrm{MgO}$ tunnel barrier of 6 and 10 monolayers thickness, respectively. b, The corresponding Seebeck coefficients for parallel $\left(S_{\mathrm{P}}\right)$ and antiparallel $\left(S_{\mathrm{AP}}\right)$ magnetization alignment. Taken from ref. [52] (ㅇ 2013 American Physical Society) and edited.
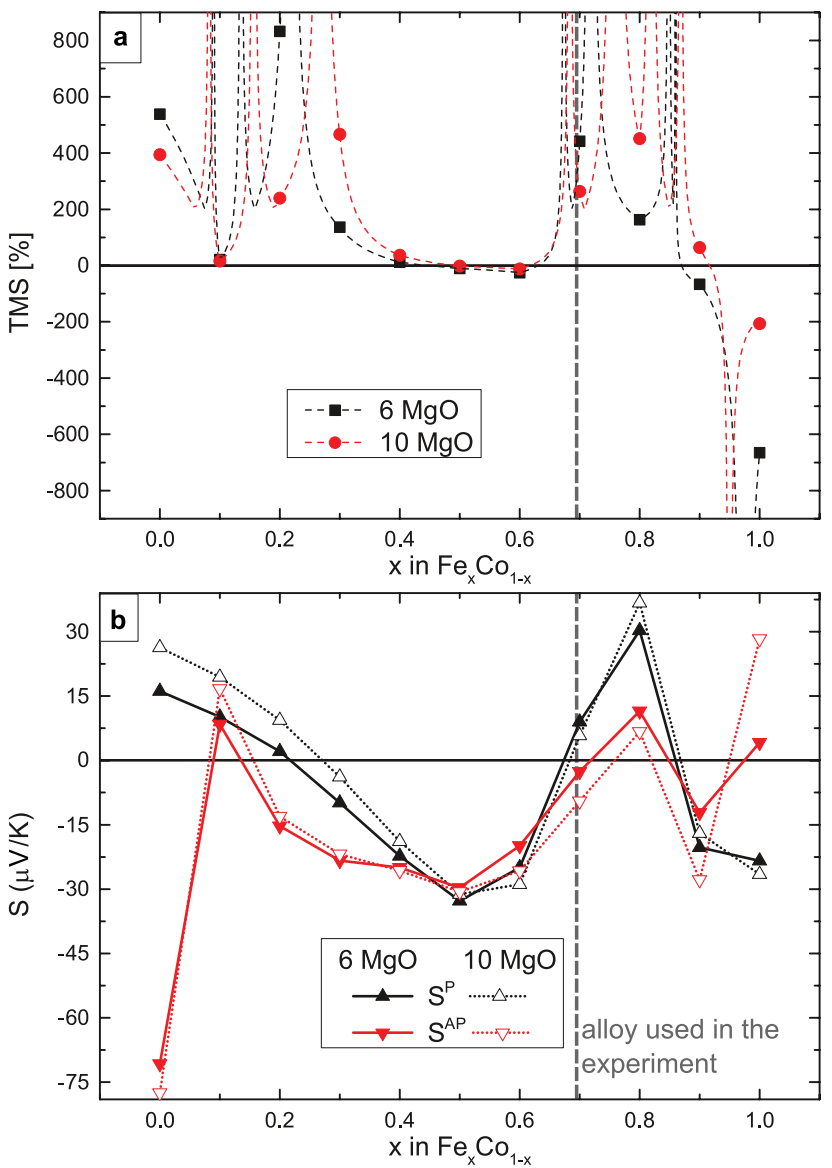

results of the calculations carried out by Heiliger et al. for Co-Fe alloy compositions ranging from pure Co $(x=0)$ to pure Fe $(x=1)$ in steps of $\Delta x=0.1$. The TMS ratios and Seebeck coefficients shown in Fig. 5.1 are the room temperature values. There is no observable trend in the dependence of TMS ratios on Co-Fe composition (Fig. 5.1(a)). The divergences of the TMS ratios for several compositions are due to zero-crossings of either $S_{\mathrm{P}}$ or $S_{\mathrm{AP}}$ in Fig. 5.1(b). For Fe contents less than $30 \%$, as well as between $70 \%$ and $80 \%$, large, positive TMS ratios are obtained. Fe contents between $40 \%$ and $60 \%$, on the other hand, lead to very small TMS ratios that exhibit a sign change at around $50 \%$ Fe content. Finally, the TMS ratio of pure Fe is negative and large. In Fig. 5.1(b) it can be seen that the Seebeck coefficients also do not show a clear dependence on the Co-Fe composition. Both, $S_{\mathrm{P}}$ and $S_{\mathrm{AP}}$ oscillate between positive and negative values, so that even cases exist, in which $S_{\mathrm{P}}$ is positive whereas $S_{\mathrm{AP}}$ is negative, and vice versa. Several zero-crossings occur for both magnetization alignments when the Fe content is varied from $0 \%$ to $100 \%$ in which the TMS ratio diverges due to its definition (eq. (1.25)). The change 

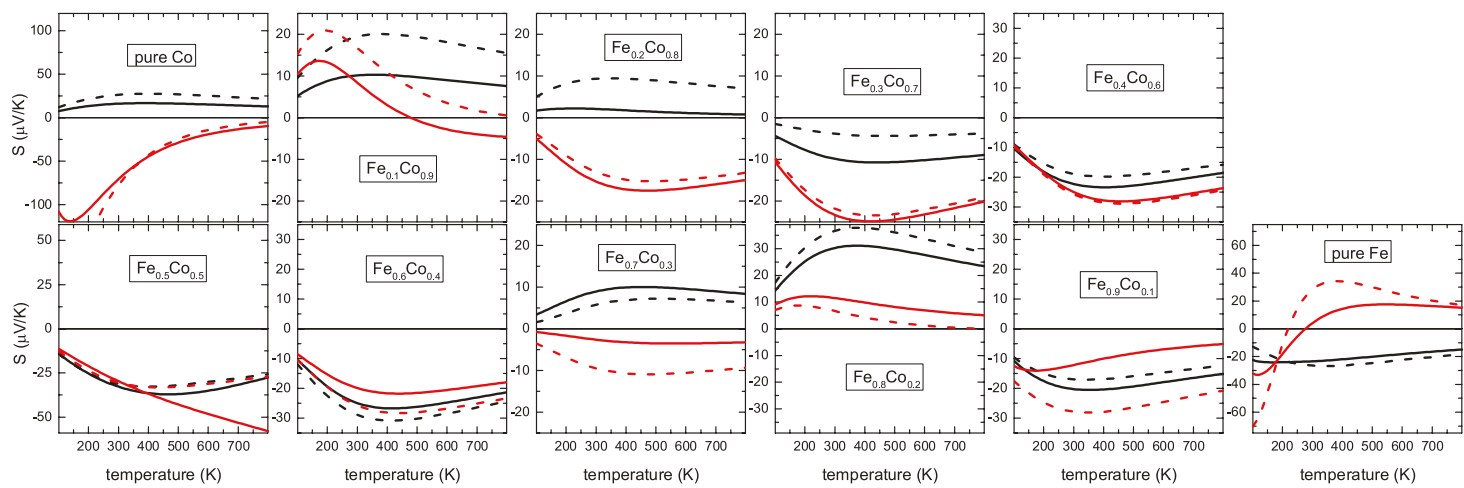

Figure 5.2: Temperature dependence of the Seebeck coefficients for different Co-Fe alloys. Results of ab initio calculations for temperatures between $100 \mathrm{~K}$ and 800 K. Seebeck coefficients are shown for parallel (black) and antiparallel (red) magnetization alignment, respectively. Dashed lines are used for a 10 monolayer (ML) $\mathrm{MgO}$ barrier, whereas solid lines are used for $6 \mathrm{ML} \mathrm{MgO}$. The Co-Fe compositions are the same as in Fig. 5.1. Taken from ref. [52]. ㅇ 2013 American Physical Society.

in barrier thickness leads only to minor changes in the behavior of the Seebeck coefficients.

Heiliger et $a l .^{52}$ also calculated the temperature dependence of the Seebeck coefficients for the considered Co-Fe compositions, as shown in Fig. 5.2. The results suggest that the Seebeck coefficients change only marginally at temperatures above $400 \mathrm{~K}$ for most of the Co-Fe compositions. A sign change of one of the Seebeck coefficients occurs for $10 \%$ and $80 \%$ Fe content and for pure Fe. A crossing of $S_{\mathrm{P}}$ and $S_{\mathrm{AP}}$ is also predicted for several compositions within the considered temperature range of $100 \mathrm{~K}$ to $800 \mathrm{~K}$.

The experimental realization of MTJs with different Co-Fe-B compositions is, however, challenging: Targets with the desired compositions need to be tested, the stoichiometry of the Co-Fe-B film on the sample needs to be analyzed, growth conditions have to be optimized and argon ion milling parameters determined. But, on the experimental side, another approach is conceivable. Since the change of CoFe-B composition leads to a shift of the Fermi level and hence to a change in the asymmetry of the transmission function, this shift can also be realized by applying an external voltage to one of the electrodes.

\subsubsection{Sample preparation}

The sample discussed in this section contains pseudo-spin-valve junctions made of a layer stack, similar to those on the samples discussed in chapters 2 and 4 . The layer stack was fabricated under UHV conditions (base pressures around $5 \times 10^{-10} \mathrm{mbar}$ ) 
in two interconnected chambers. In the first chamber, the Ta and Co-Fe-B layers were deposited by magnetron sputtering. The $\mathrm{MgO}$ tunnel barrier as well as the $\mathrm{Ru}$ capping layer were deposited in the second chamber by e-beam evaporation. The layer stack was deposited on a single crystalline $\mathrm{MgO}$ substrate and is composed as follows: $\mathrm{MgO}$ substrate / $10 \mathrm{~nm} \mathrm{Ta} \mathrm{/} 2.5 \mathrm{~nm} \mathrm{Co}_{20} \mathrm{Fe}_{60} \mathrm{~B}_{20} / 1.7 \mathrm{~nm} \mathrm{MgO} \mathrm{/} 5.4 \mathrm{~nm}$ $\mathrm{Co}_{20} \mathrm{Fe}_{60} \mathrm{~B}_{20} / 5.0 \mathrm{~nm} \mathrm{Ta} / 3.0 \mathrm{~nm} \mathrm{Ru}$. In subsequent e-beam lithography and argon ion milling steps, which were conducted at Bielefeld University, $125 \mathrm{~nm} \mathrm{Si}_{3} \mathrm{~N}_{4}$ were deposited to insulate the junction pillar and $15 \mathrm{~nm}$ Ta plus $92 \mathrm{~nm}$ Au were added afterwards as top contact layers which were patterned as bond pads.

A detailed investigation of the TMR and TMS effect of this sample can be found in Tim Eggebrecht's master's thesis ${ }^{79}$. In the following, the discussion will focus on the bias-dependent measurements of the thermocurrent.

\subsubsection{Experimental setup}

The setup used for the experiments discussed in this section is depicted in Fig. 5.3. The optical setup (Fig. 5.3(a)) is unchanged from the one described already in

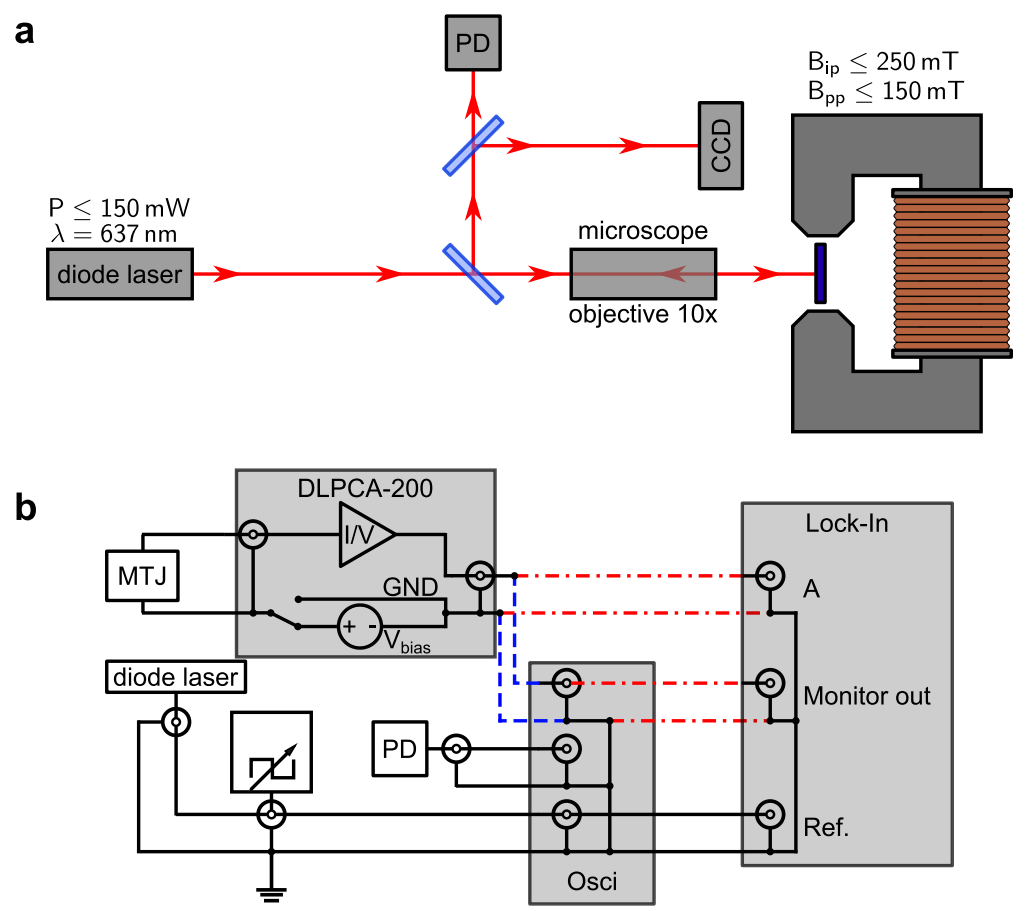

Figure 5.3: Experimental setup for TMS measurements with bias voltage.

a, Optical setup as in chapter 3. b, Electrical setup as in chapter 3 in which the precision voltage amplifier is exchanged for a transimpedance amplifier. This amplifier can be either set to ground, or a bias voltage can be applied to the MTJ. 
chapter 3. The former electrical setup (chapter 3, Fig. 3.1 (b)), however, was modified to facilitate the detection of thermoelectric effects in the MTJ while a $\mathrm{DC}$ voltage is applied to the latter. Since the laser is modulated at a frequency of $f=1.5 \mathrm{kHz}$, the most straightforward way to determine the thermoelectric effects occurring in the MTJ is to observe the current signal at the frequency $f$. This part of the total electric current originates from the laser heating modulated at this frequency. For this reason, the precision voltage amplifier used in Fig. 3.1 was exchanged for a precision transimpedance amplifier (DLPCA-200, femto GmbH) which takes a current as input and outputs an amplified voltage proportional to this current (Fig. 5.3b). This amplifier further allows the application of a DC voltage to the MTJ. Alternatively, it can be set to measure against ground potential. In addition, the amplifier output is $\mathrm{AC}$ coupled so that it does not detect the electric current caused by the DC bias voltage. A transimpedance gain ranging between $1 \times 10^{3} \mathrm{VA}^{-1}$ and $1 \times 10^{11} \mathrm{VA}^{-1}$ can be chosen, depending on the magnitude of the current signal. As indicated in Fig. 5.3b, the output of the transimpedance amplifier is connected to a lock-in amplifier which is used for data acquisition.

\subsubsection{Estimate of Peltier and Thomson effects}

The application of a DC bias voltage during the thermoelectrical characterization leads to a DC current flowing through the MTJ, concurrently to a temperature gradient created by the laser heating. In this scenario, two other thermoelectric effects come into play: the Peltier and the Thomson effect that were introduced in sections 1.1.2 and 1.1.3, respectively. In the following, the coefficients for both effects will be estimated using the theoretical predictions described earlier in section 5.1, as well as experimental results.

To calculate the Peltier coefficient, it is necessary to know the Seebeck coefficient. Seebeck coefficients for $\mathrm{Co}-\mathrm{Fe}-\mathrm{B} / \mathrm{MgO}$ tunnel junctions were presented in chapters 2 and 3 . They range from $100 \mu \mathrm{VK}^{-1}$ to $S_{\max }=770 \mu \mathrm{VK}^{-1}$. Due to an underestimation of the temperature gradients in the MTJs caused by unknown thin film thermal conductivities and interface resistances, these values represent an upper limit for the Seebeck coefficients. Ab initio calculations (Figs. 5.1 and 5.2) revealed Seebeck coefficients in the order of $S_{\min }=30 \mu \mathrm{V} \mathrm{K}^{-1}$. Because of this uncertainty, the Peltier coefficient is calculated according to eq. (1.17) for both extreme cases. The temperature at the $\mathrm{MgO}$ barrier might reach $T=400 \mathrm{~K}$ for higher laser powers. The minimum and maximum Peltier coefficients thus amount to:

$$
\begin{aligned}
\Pi_{\max } & =S_{\max } T \approx 308 \mathrm{mV}, \\
\Pi_{\min } & =S_{\min } T \approx 12 \mathrm{mV} .
\end{aligned}
$$

The measurements, which will be presented in the following, were carried out at a maximum bias voltage of $V_{\max }=300 \mathrm{mV}$. The resistance of the MTJ for parallel 
magnetization alignment can be taken from Fig. 5.4 and amounts to $R_{\mathrm{P}}=6 \mathrm{k} \Omega$. This leads to a maximum current of $I_{\max }=50 \mu \mathrm{A}$. The heat current $I_{Q}$ generated by the Peltier effect can now be calculated using eq. (1.16), the electric current $I$ and the Peltier coefficients:

$$
\begin{aligned}
I_{Q}^{\max } & =\Pi_{\max } I_{\max }=16 \mu \mathrm{W}, \\
I_{Q}^{\min } & =\Pi_{\min } I_{\max }=0.6 \mu \mathrm{W} .
\end{aligned}
$$

This heat can be neglected in the following experiments, in which laser powers of $150 \mathrm{~mW}$ (and focussed to approximately the MTJ diameter) are incident on the MTJ.

The change of the Seebeck coefficient with temperature enters eq. (1.20) for the Thomson coefficient. Since the exact temperature dependence of the Seebeck coefficient cannot be deduced from experiments so far, the results of the ab initio calculations shown in Fig. 5.2 are taken into account. As can be seen in the figure, for most alloy compositions, the Seebeck coefficient at $T=400 \mathrm{~K}$ is nearly constant, thus $\frac{\mathrm{d} S}{\mathrm{~d} T} \approx 0$. As a consequence, the Thomson coefficient $\beta=T \frac{\mathrm{d} S}{\mathrm{~d} T} \approx 0$. Therefore, the Thomson heat will be also neglected in the discussion of the following experiments.

\subsubsection{First results}

Prior to the evaluation of the thermocurrent measurements, the bias dependent TMR measurements have to be analyzed. Fig. 5.4a shows two series of TMR measurements that were performed at a variety of bias voltages on a $6 \mu \mathrm{m} \times 4 \mu \mathrm{m}$
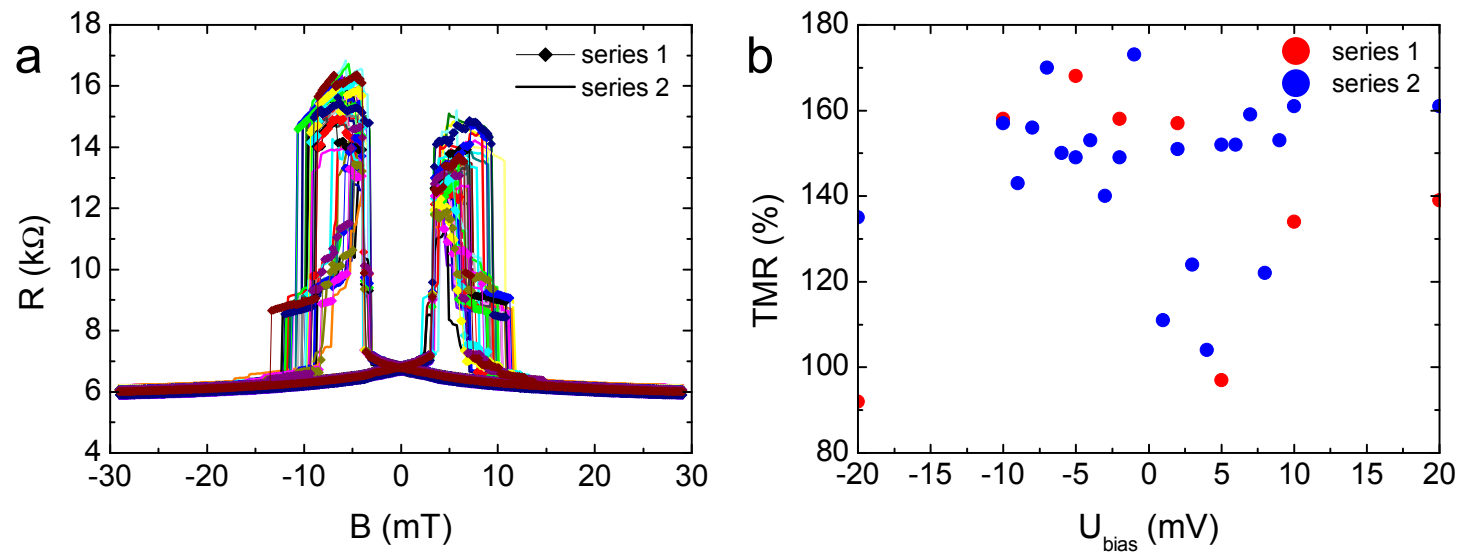

Figure 5.4: Dependence of TMR ratio on bias voltage. a, TMR measurements performed at different bias voltages. The resistance of parallel magnetization alignment is found to be constant, $R_{\mathrm{P}} \approx 6 \mathrm{k} \Omega$. b. TMR ratios calculated from the curves shown in $\mathbf{a}$ as function of bias voltage. No clear dependence on the bias voltage can be identified. 


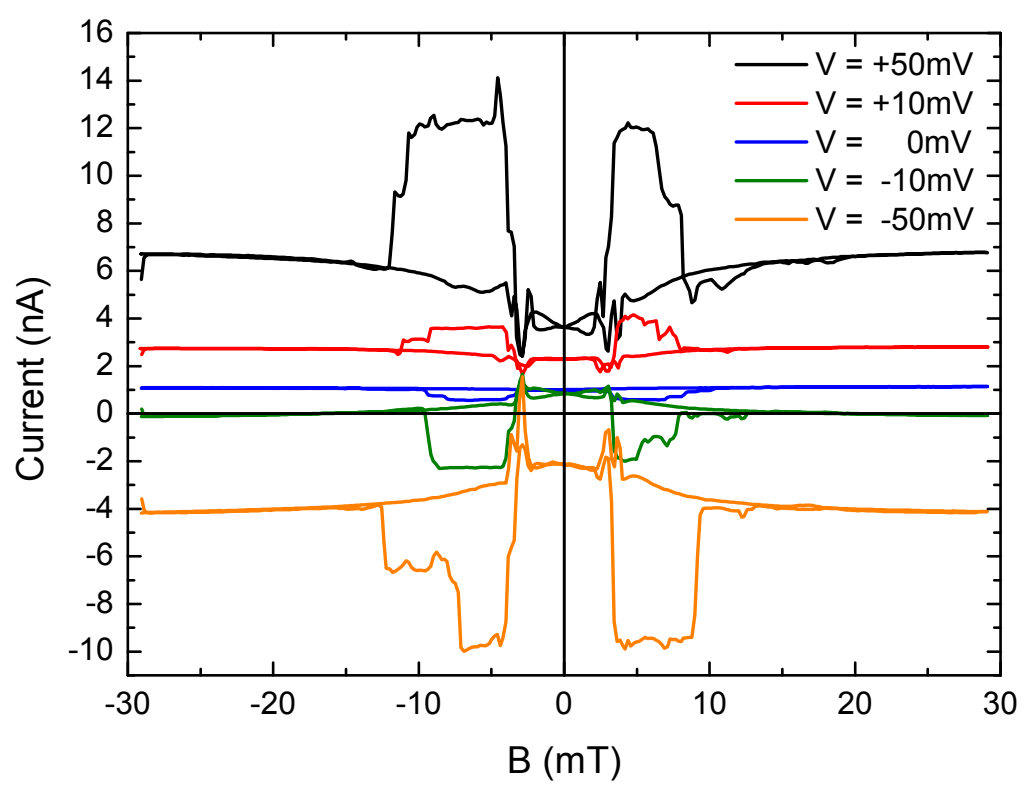

Figure 5.5: Thermocurrent measurements at different bias voltages. Measurements at selected voltages between $-50 \mathrm{mV}$ and $50 \mathrm{mV}$ are depicted. The current signal is detected by the lock-in amplifier at the laser heating modulation frequency of $1.5 \mathrm{kHz}$.

elliptical MTJ. The curves indicate several switching fields at which the magnetization alignment changes from antiparallel back to parallel. A possible reason for this behavior is the existence of several magnetic domains in the two ferromagnetic layers of the MTJ. All curves have a resistance $R_{\mathrm{P}} \approx 6 \mathrm{k} \Omega$ for the parallel magnetization state in common. The TMR ratios calculated from these curves are shown in Fig. 5.4b as a function of bias voltage. It can be seen that the TMR ratios are distributed randomly between $170 \%$ and $90 \%$ and that no clear dependence on the applied bias voltage can be identified. A possible reason for this broad distribution is the above mentioned multi-domain switching behavior of this MTJ. For this reason, an average TMR ratio of $145 \%$ is used for further analysis. Given this TMR ratio and a resistance of $6 \mathrm{k} \Omega$ in the parallel state, the average resistance in antiparallel state becomes $R_{\mathrm{AP}} \approx 14.7 \mathrm{k} \Omega$.

A selection of thermocurrent measurements is shown in Fig. 5.5. The lock-in amplifier was triggered such that the thermocurrent signal appears at the X (inphase) input of the lock-in amplifier. Thus, a positive current leads to a positive $\mathrm{X}$ signal and vice versa. Further, to obtain the curves plotted in Fig. 5.5, the voltages measured by the lock-in amplifier were converted to currents through division by the transimpedance gain of the preamplifier. A test without laser heating and a DC bias voltage of up to $300 \mathrm{mV}$ revealed only a negligible current signal around $0.03 \mathrm{nA}$. This verifies that only currents caused by the laser heating are detected. 
At $50 \mathrm{mV}$ bias voltage, a positive thermocurrent is observed. The current is larger for antiparallel magnetization alignment of the electrodes. The same situationwith a smaller difference between the voltages in parallel and antiparallel state - is observed at $10 \mathrm{mV}$, whereas the thermocurrent is larger in parallel state when the measurement is performed without bias voltage. At $-10 \mathrm{mV}$, the thermocurrent for parallel alignment is almost shifted to zero and a negative current is observed in antiparallel state. Finally, at $-50 \mathrm{mV}$ bias voltage, negative thermocurrents are observed for both magnetization alignments, of which the magnitude is larger for the antiparallel configuration.

The Seebeck voltages generated during laser heating can be derived from the thermocurrent measurements presented in Fig. 5.5 and the TMR measurements depicted in Fig. 5.4. According to eq. (3.1) in section 3.4.1, the current $I$ is given by

$$
I=G V+G S \Delta T \text {. }
$$

The Seebeck voltage $(S \Delta T)$ thus becomes in general:

$$
S \Delta T=\frac{1}{G} I-V .
$$

Due to the measurement technique, the DC bias voltage $V$ is not detected in the thermocurrent measurements. In addition, substituting the conductance $G$ with the resistance $R_{\mathrm{P} / \mathrm{AP}}$ of the two magnetization alignments, yields a relation that connects the thermocurrents and resistances found in parallel and antiparallel state to the respective Seebeck voltages:

$$
(S \Delta T)_{\mathrm{P} / \mathrm{AP}}=R_{\mathrm{P} / \mathrm{AP}} I_{\mathrm{P} / \mathrm{AP}}
$$

The data in Fig. 5.6a are calculated from thermocurrent and TMR measurements using eq. (5.5). On the larger voltage scale up to $\pm 200 \mathrm{mV}$, the Seebeck voltages depend linearly on the applied bias voltage. Both slopes, for parallel and antiparallel magnetization configuration, are positive, whereupon the magnitude of the slope is larger for the latter configuration. For small bias voltages below $10 \mathrm{mV}$, the behavior of the Seebeck voltages exhibits more structure (inset of Fig. 5.6a): The voltages in parallel and antiparallel state are almost equal and nearly constant for bias voltages between $0 \mathrm{mV}$ and $5 \mathrm{mV}$. Similarly, the voltages are also nearly constant between $0 \mathrm{mV}$ and $-5 \mathrm{mV}$, yet the difference between the Seebeck voltages observed in parallel and antiparallel state is large. At bias voltages higher than $\pm 5 \mathrm{mV}$, the linear behavior starts to develop.

The different slopes of the Seebeck voltages lead to negative TMS ratios for positive bias voltages that decrease in magnitude when the bias voltage approaches zero. Between $0 \mathrm{mV}$ and $-10 \mathrm{mV}$ the sign of the TMS ratio changes and due to the zero crossing of the parallel state Seebeck voltage at approximately $-10 \mathrm{mV}$, a TMS ratio of $7000 \%$ is obtained. With further increasing bias voltages, the TMS ratio drops again to a nearly constant value of $250 \%$. 


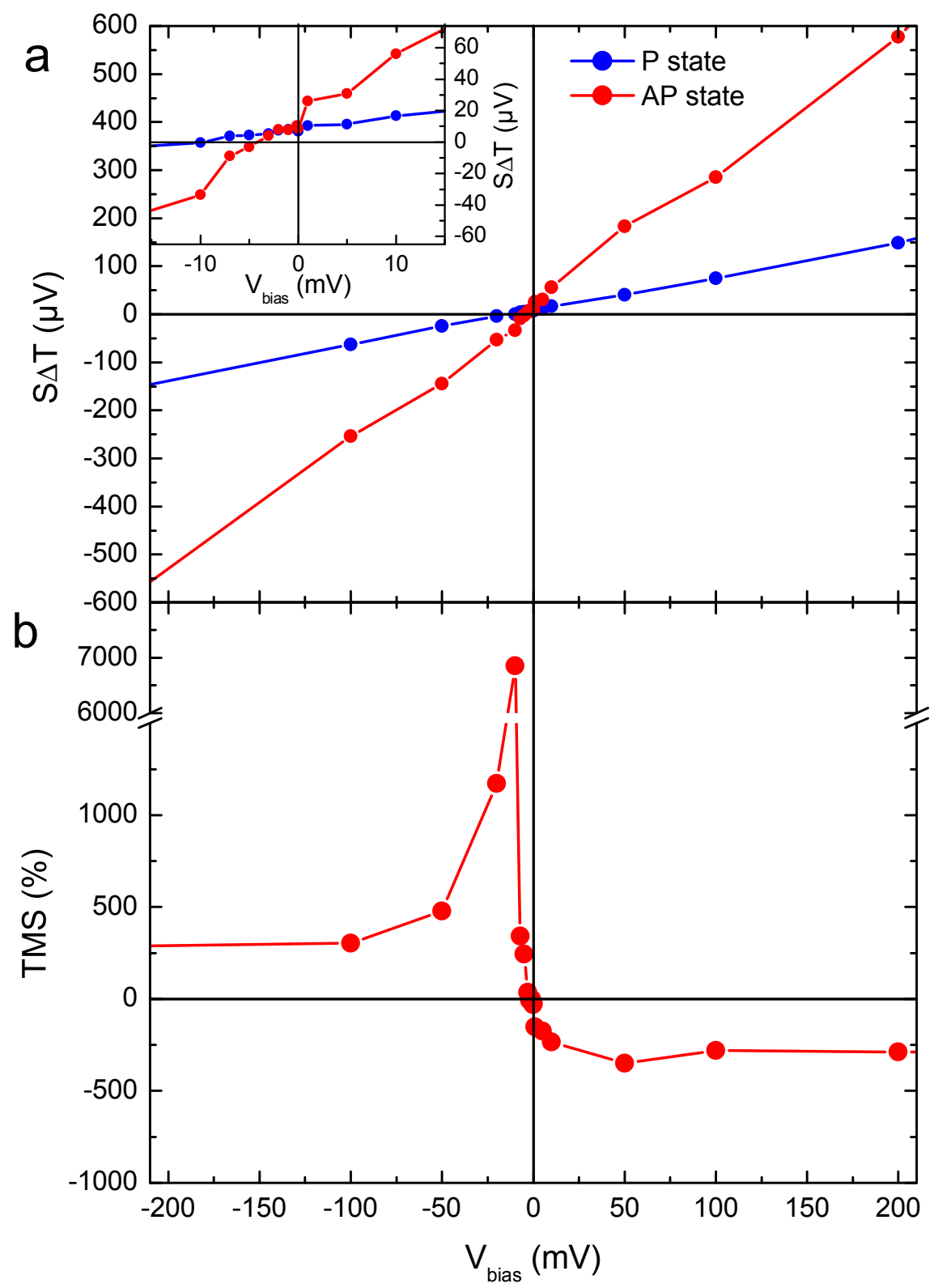

Figure 5.6: Bias voltage dependence of the TMS effect. a, Seebeck voltages extracted from thermocurrent and TMR measurements at different bias voltages. b, TMS ratio calculated from the Seebeck voltages shown above.

\subsubsection{Discussion}

The observations made in this experiment deviate from the theoretical predictions for different Co-Fe alloys introduced at the beginning of section 5.1. The ab initio calculations predict that the change of Fe content from $x=0$ to $x=1$ in a 
$\mathrm{Fe}_{x} \mathrm{Co}_{1-x}$ alloy leads to shifts of several hundred meV of features in the transmission function (Fig. 4 in ref. [52]). This energy range is also covered by the bias voltage measurements presented here. However, whereas the Seebeck voltages were found to be linearly dependent on the applied bias voltage, the calculations predict a rather oscillatory behavior of the Seebeck coefficients as a function of alloy composition.

On the other hand, the Co-Fe composition used in the experiment is close to $x=0.7$ in Fig. 5.1. Close to this composition, the calculations also predict a linear behavior of the Seebeck coefficients. The slopes are positive, in the experiment as well as in the $a b$ initio calculations.

Furthermore, the changes in Seebeck coefficients caused by modifications of the alloy composition cannot be compared directly to the bias voltage dependent measurements. Although both methods lead to shifts of the Fermi level in the ferromagnetic electrodes of the MTJ, the application of a bias voltage changes only the Fermi level of one electrode, whereas a change of alloy composition in both electrodes also leads to a shift in Fermi level in the two.

As a consequence, further $a b$ initio calculations are currently underway that will allow a direct comparison between theory and experiment. On the experimental side, further measurement series have to be carried out to improve the experimental statistics. These experiments should include power dependent measurements performed at each bias voltage to obtain a qualitative insight into the temperature dependence of the Seebeck coefficients (similar to Fig. 5.2).

The most interesting outcome of this measurement series is that a bias voltage can be used in the experiment to tailor the response of the Seebeck voltage, both in voltage sign and magnitude. Especially the measurement at $-10 \mathrm{mV}$, in which the Seebeck voltage is close to zero for parallel alignment, presents the possibility of building a thermoelectric device that can be magnetically turned on and off.

\subsection{Tunnel magneto-Seebeck effect in Heusler compound tunnel junctions}

The previous chapters dealt with investigations of the effect in $\mathrm{Co}-\mathrm{Fe}-\mathrm{B} / \mathrm{MgO}$ magnetic tunnel junctions. However, another interesting class of materials exists which fulfills the demands for a high tunnel magneto-Seebeck effect: the class of half metals. While the ferromagnetic transition metals Fe and Co exhibit spin-polarizations $P$ below $60 \%$-which is consistent with the maximum observed TMR ratios below $100 \%$ in MTJs with alumina barrier, according to the Jullière model ${ }^{16}$ —a spin-polarization of $100 \%$ can be achieved in half metals. This is due to the spindependent electronic structure of these materials, which is metallic for one type of spins, whereas it contains a band gap for the opposite spin direction and thus acts as an insulator for the latter spins. Such a high intrinsic spin-polarization has the advantage that no special choice of tunnel barrier material is needed to build MTJs 
with very high TMR ratios and tunnel magneto-Seebeck effects.

An all-optical determination of the spin polarization of a selection of half metals was done by Müller et $a l .{ }^{45}$ and Mann et al. ${ }^{80}$. The results obtained by Mann et al. ${ }^{80}$ are depicted in Fig. 5.7. The spin-polarization of the half metal $\mathrm{CrO}_{2}$ amounts to $99 \%$ in the experiment and is the highest of the investigated materials. The Heusler compounds $\mathrm{CO}_{2} \mathrm{FeAl}, \mathrm{Co}_{2} \mathrm{MnGe}$ and $\mathrm{Co}_{2} \mathrm{MnSi}$, belonging to the class of half metals, showed spin polarizations between $66 \%$ and $86 \%$. These values are still far from the $99 \%$ of $\mathrm{CrO}_{2}$, but are already significantly higher than the spin-polarization of the Co-Fe-B alloy $(P=65 \%)$ and of the transition metal ferromagnets CoFe $(P=55 \%)$, permalloy $\left(\mathrm{Ni}_{81} \mathrm{Fe}_{19}, P=48 \%\right)$ and $\mathrm{Ni}(P=45 \%)$, which are also shown in Fig. 5.7 for comparison.

Consequently, first experiments with magnetic tunnel junctions employing the Heusler compound $\mathrm{Co}_{2} \mathrm{FeSi}$ as electrode material were carried out in the course of this thesis. The following subsections describe the sample preparation and discuss the dependence of the tunnel magneto-Seebeck effect on modulation frequency and laser power. In addition, thermocurrent measurements were carried out as a function of applied laser power. Further, position-dependent measurements are discussed, in which the laser spot was moved away from the contacted MTJ.

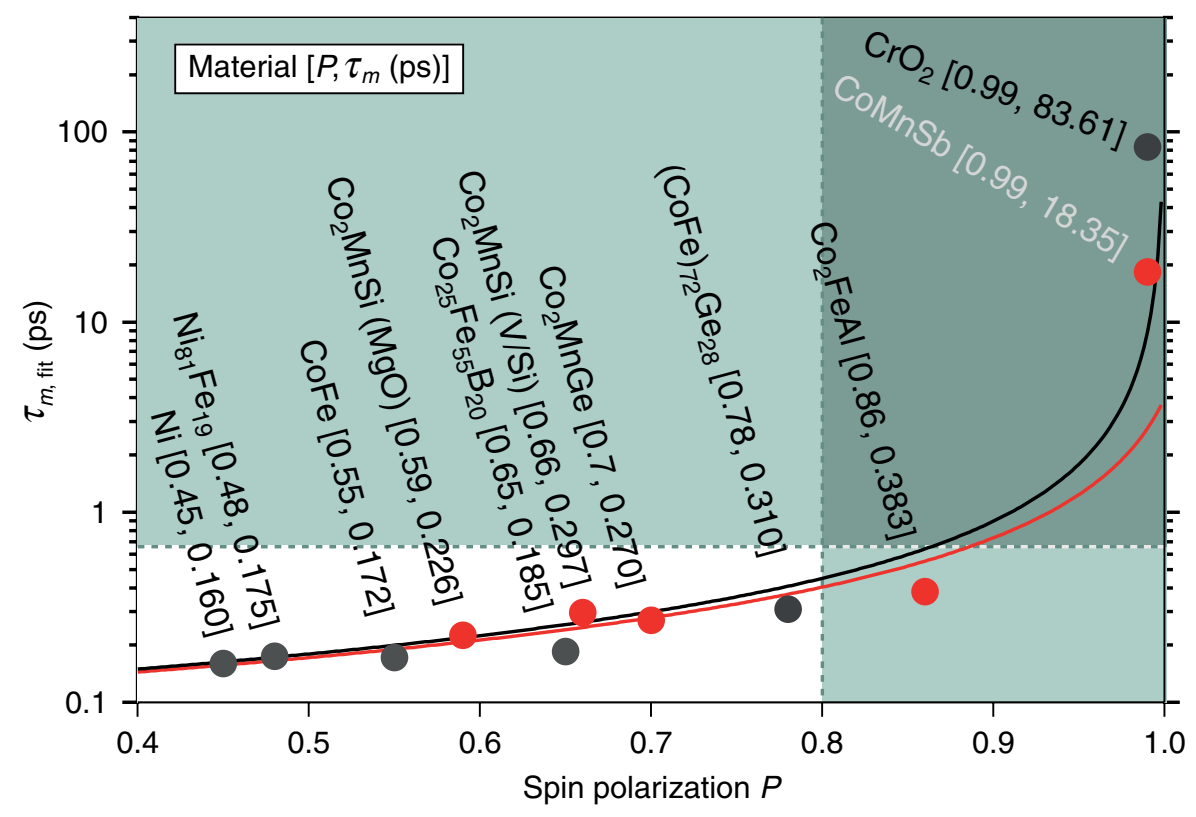

Figure 5.7: Spin polarization of selected half metals. The demagnetization time and spin-polarization are determined from all-optical pump-probe experiments. The half-metallic behavior is modeled theoretically and results in the functional dependence $\tau_{\mathrm{m}}(P)$ given by the black and red lines. Taken from ref. [80]. 


\subsubsection{Sample preparation and TMR}

The sample investigated in the following was prepared under ultra high vacuum conditions on a single-crystalline $\mathrm{MgO}$ substrate. The layers were sputter deposited on the substrate at room temperature. The layer stack is as follows (see also Fig. 5.8a): $\mathrm{MgO}$ substrate / $5 \mathrm{~nm} \mathrm{MgO} \mathrm{/} 5 \mathrm{~nm} \mathrm{Cr} / 20 \mathrm{~nm} \mathrm{Co}_{2} \mathrm{FeSi} / 2 \mathrm{~nm} \mathrm{MgO}$ / $5 \mathrm{~nm} \mathrm{Co}{ }_{70} \mathrm{Fe}_{30} / 10 \mathrm{~nm} \mathrm{Mn}_{83} \mathrm{Ir}_{17} / 25 \mathrm{~nm} \mathrm{Ru}$. After deposition, the sample was post-annealed in a separate vacuum chamber for $1 \mathrm{~h}$ at a temperature of $325^{\circ} \mathrm{C}$. An external magnetic field of $650 \mathrm{mT}$ was applied during annealing. The $\mathrm{Mn}_{83} \mathrm{Ir}_{17}$ layer pins the magnetization of the $\mathrm{Co}_{70} \mathrm{Fe}_{30}$ layer by exchange bias and leads to the minor loop shown in Fig. 5.8b.

Elliptical MTJs of $6 \mu \mathrm{m} \times 4 \mu \mathrm{m}, 4 \mu \mathrm{m} \times 2 \mu \mathrm{m}$ and $2 \mu \mathrm{m} \times 1 \mu \mathrm{m}$ in lateral size were prepared out of the layer stack by electron beam lithography and argon ion milling down to the middle of the $\mathrm{Co}_{2} \mathrm{FeSi}$ layer. The long axis of the ellipses was chosen to be parallel to the direction of magnetic field during annealing to strengthen the magnetic easy axis of the two ferromagnetic electrodes through the shape anisotropy. In subsequent steps, tantalum oxide was deposited around the MTJ pillar as insulation, and additionally added $5 \mathrm{~nm} \mathrm{Ta} / 70 \mathrm{~nm} \mathrm{Au}$ were patterned into bond pads on top of the MTJ, again by e-beam lithography and argon ion milling.

TMR measurements that were taken throughout the electro-optical characteriza-

a

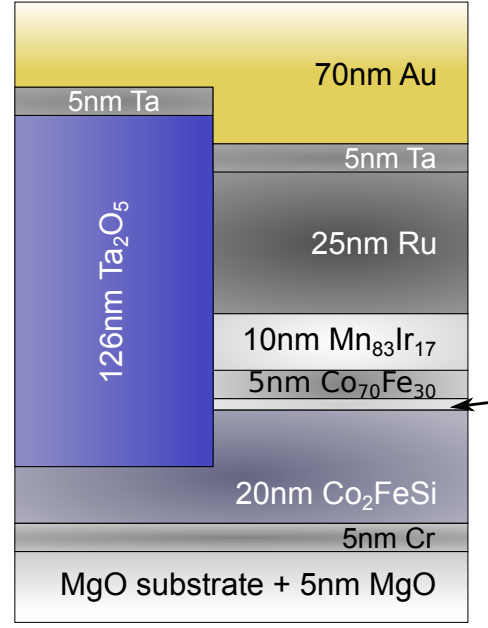

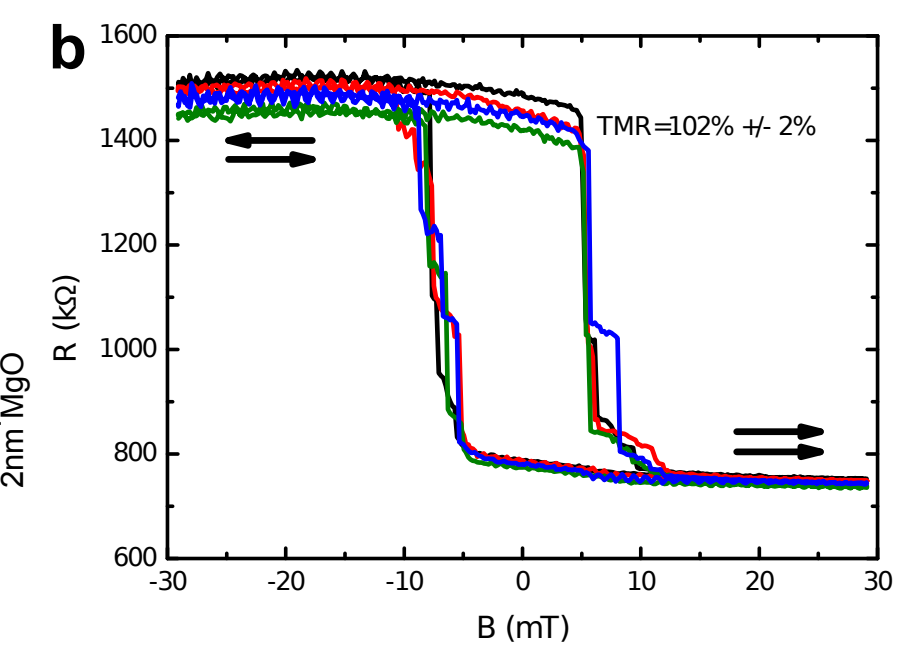

Figure 5.8: Layer stack of the Heusler compound sample and TMR measurements. a, Layer stack of the MTJ containing the Heusler compound $\mathrm{Co}_{2} \mathrm{FeSi}$ as one ferromagnetic electrode and a CoFe alloy as the second ferromagnet, separated by a $2 \mathrm{~nm} \mathrm{MgO}$ barrier. b, TMR measurements taken throughout the electro-optical characterization of the MTJ. The arrows mark the magnetization alignment of the two electrodes. 
tion process are shown in Fig. 5.8b. The TMR ratio is at $102 \%$ already quite high, but still significantly lower than TMR ratios observed in $\mathrm{Co}-\mathrm{Fe}-\mathrm{B} / \mathrm{MgO}$ samples.

\subsubsection{Frequency dependence of the Seebeck voltage}

The first measurements were performed with a diode laser modulation frequency of $1.5 \mathrm{kHz}$, as described in section 3.2 . However, the Seebeck voltages recorded by the oscilloscope, which are shown in Fig. 5.9a, do not show the expected squarewave form. As a consequence, oscilloscope traces were taken at $713 \mathrm{~Hz}$ and $77 \mathrm{~Hz}$ modulation frequency.

It can be seen in Fig. 5.9a that a saturation of the Seebeck voltage is already visible at $713 \mathrm{~Hz}$ for parallel electrode alignment. For the antiparallel alignment, in contrast, the Seebeck voltage has not yet reached its saturation value. At $77 \mathrm{~Hz}$, the Seebeck voltages are saturated for parallel and antiparallel alignment. Fig. 5.9b shows TMS measurements carried out at $1500 \mathrm{~Hz}$ and $77 \mathrm{~Hz}$. Due to the slow

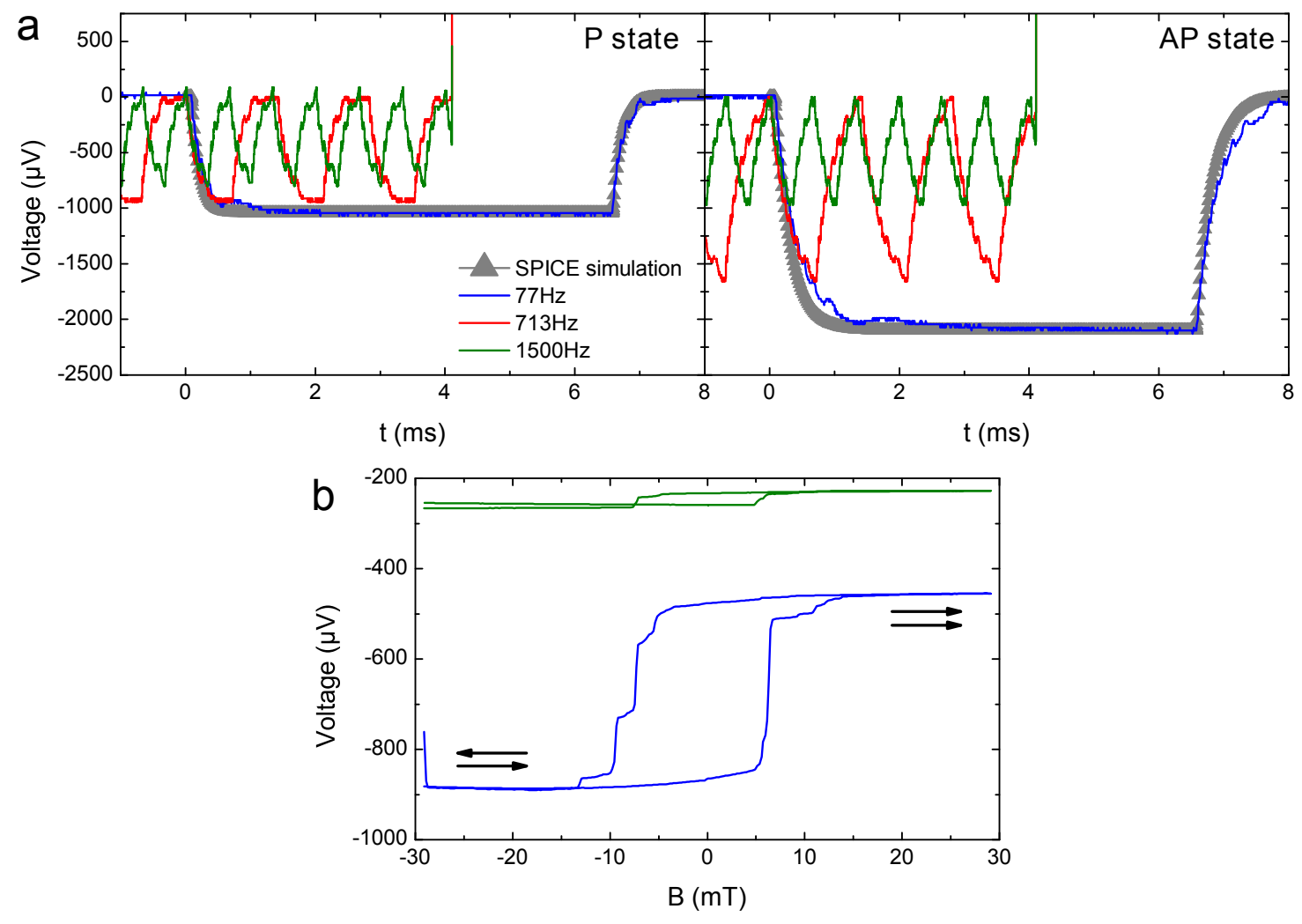

Figure 5.9: Frequency dependence of the Seebeck voltage. a, Time-dependent Seebeck voltages recorded with an oscilloscope for parallel and antiparallel magnetization alignment. The laser was modulated with $77 \mathrm{~Hz}, 713 \mathrm{~Hz}$ and $1500 \mathrm{~Hz}$. b, TMS measurements taken with $77 \mathrm{~Hz}$ and $1500 \mathrm{~Hz}$, respectively. 
response of the Seebeck voltage, not only the voltages recorded by the lock-in amplifier are much smaller at $1500 \mathrm{~Hz}$, but also the TMS.

The rise and fall times of the Seebeck voltage are larger by more than an order of magnitude than those found in the $\mathrm{Co}-\mathrm{Fe}-\mathrm{B} / \mathrm{MgO}$ samples investigated in chapter 3 . However, there are differences between the Heusler compound sample regarded in this chapter and the Co-Fe-B/MgO samples: First, the thickness of the MgO barrier amounts to $2 \mathrm{~nm}$, whereas the barrier thickness of the samples used in chapter 3 was $1.5 \mathrm{~nm}$. Second, $6 \mu \mathrm{m} \times 4 \mu \mathrm{m}$ ellipses with an area of $19 \mu \mathrm{m}^{2}$ were studied in the $\mathrm{Co}-\mathrm{Fe}-\mathrm{B} / \mathrm{MgO}$ case, which is larger by a factor of 3 than the area of the $4 \mu \mathrm{m} \times 2 \mu \mathrm{m}$ ellipses $\left(\right.$ area $\approx 6 \mu^{2}$ ) considered here. Both quantities change the resistance and the capacitance of the MTJs. Tim Eggebrecht reported in his master's thesis ${ }^{79}$ that slight changes in the temporal evolution of the Seebeck voltage could be explained by changes in resistance and capacitance of the MTJ and, hence, by a different time constant of the circuit. For this reason, SPICE simulations were carried out to check if the different timescales observed in the oscilloscope traces of the Heusler compound MTJ can completely be attributed to a change in the time constant of the circuit. The simulation parameters were taken from table 3.3 in chapter 3 , except the capacitance and resistance of the MTJ and the Seebeck voltages. According to Fig. $5.8 \mathrm{~b}$, the resistances $R_{\mathrm{P}} \approx 744 \mathrm{k} \Omega$ and $R_{\mathrm{AP}} \approx 1481 \mathrm{k} \Omega$ were taken for parallel and antiparallel magnetization alignment, respectively. Given an MTJ area $A \approx 6 \mathrm{~mm}^{2}$, a $\mathrm{MgO}$ thickness of $d=2 \mathrm{~nm}$ and a permittivity of $\mathrm{MgO}$ of $\varepsilon_{r}^{\mathrm{MgO}}=9.6$ (ref. [81]), the capacitance of the Heusler compound MTJ becomes:

$$
C_{\mathrm{MTJ}}=\varepsilon_{0} \cdot \varepsilon_{r}^{\mathrm{MgO}} \cdot \frac{A}{d} \approx 0.267 \mathrm{pF}
$$

The Seebeck voltages for parallel and antiparallel alignment were determined from Fig. 5.9a and amount to $V_{\mathrm{P}} \approx-1043 \mu \mathrm{V}$ and $V_{\mathrm{AP}} \approx-2100 \mu \mathrm{V}$, respectively. The results of the SPICE simulations with these parameters are depicted in Fig. 5.9a as gray triangles and are in good agreement with the Seebeck voltages observed in the experiment (blue lines). Therefore, no evidence for a change in heat transport is found in MTJs containing Heusler compounds, when compared to Co-Fe-B/MgO MTJs.

Nevertheless, one striking difference between the time-dependent Seebeck voltages of $\mathrm{Co}-\mathrm{Fe}-\mathrm{B} / \mathrm{MgO}$ and Heusler compound MTJs can be accounted for: Whereas the Seebeck voltages of the samples discussed in chapter 3 and of the in-plane anisotropic samples investigated in ref. [79] were all positive, the MTJs containing the Heusler compound $\mathrm{Co}_{2} \mathrm{FeSi}$ as one of the ferromagnetic electrodes show a negative Seebeck voltage. This means that these MTJs possess a positive Seebeck coefficient, which is for example found in semiconductors in which the electric properties are dominated by hole transport. 


\subsubsection{Dependence on applied laser power}

In the following, measurements of the tunnel magneto-Seebeck effect are presented which were carried out at a variety of laser powers. As discussed in detail in the supplementary information of the nature materials article (ref. [49], appendix A), higher laser powers lead to a raised base temperature of the MTJ as well as a higher temperature gradient across the $\mathrm{MgO}$ barrier. Thus, a qualitative study of the temperature dependence of the TMS effect is possible.

Fig. 5.10a shows measurements recorded at laser powers ranging from $1 \mathrm{~mW}$ to $150 \mathrm{~mW}$ and without laser heating. The negative voltage sign is taken from the results presented in section 5.2.2 and added to the absolute values recorded by the lock-in amplifier. As expected, the signal of the measurement without laser heating is around zero and magnetic switching is not visible. That is, no spurious voltages and noise sources couple into the experiment. Already at $1 \mathrm{~mW}$, the typical TMS minor loop can be identified in the measurement.

The Seebeck voltages measured in parallel and antiparallel magnetization state are depicted in Fig. 5.10b as a function of laser power. For both states, the absolute value of the Seebeck voltage rises linearly with increasing laser power, apart from the values observed at $150 \mathrm{~mW}$ only. The Seebeck voltages reach values of up to $-3500 \mu \mathrm{V}$ in antiparallel and $-1800 \mu \mathrm{V}$ in parallel state at $150 \mathrm{~mW}$, respectively. These values are very high when compared to the Seebeck voltages of Co-Fe-B/MgO based MTJs: Fig. 3.3a in section 3.4.2 presents a TMS measurement at $150 \mathrm{~mW}$. The Seebeck voltage amounts to roughly $90 \mu \mathrm{V}$. In Tim Eggebrecht's master's thesis $^{79}$, Seebeck voltages in the order of $10 \mu \mathrm{V}$ were observed in $\mathrm{Co}-\mathrm{Fe}-\mathrm{B} / \mathrm{MgO}$ pseudo-spin-valves. The top contact of the sample discussed in section 3.4.2 was significantly thinner, whereas the sample investigated by Eggebrecht had a slightly thicker top contact (115 nm on top of the upper ferromagnet) compared to the sample discussed here (100 nm on top of $\mathrm{Mn}_{83} \mathrm{Ir}_{17}$ layer). Hence, the voltages observed in Fig. 5.10 are compared to the results of Eggebrecht. The latter measurements were performed using a beam waist of $w_{0}=11 \mu \mathrm{m}$. In contrast, the measurements shown in Fig. 5.10 were carried out with a beam waist of $w_{0}=4 \mu \mathrm{m}$, leading to a 8 times higher laser intensity incident on the MTJ. Assuming that the linear power dependence observed by Eggebrecht is valid for higher intensities, the maximum voltages in the order of $10 \mu \mathrm{V}$ would correspond to Seebeck voltages of $\lesssim 80 \mu \mathrm{V}$ for the case of $150 \mathrm{~mW}$ laser power and a beam waist of $4 \mu \mathrm{m}$. Taking further into account that the top contact of the Heusler MTJs presented here is $15 \mathrm{~nm}$ thinner, it can still be asserted from the results of Fig. 5.10 that the Seebeck voltages of the Heusler junctions are by an order of magnitude larger than observed in Co-Fe-B/MgO based MTJs.

In addition, the extracted TMS ratios are plotted in Fig. 5.10b as a function of laser power together with the corresponding Seebeck voltages. The TMS ratios range between $90 \%$ and $100 \%$ and decrease linearly with increasing laser power. 

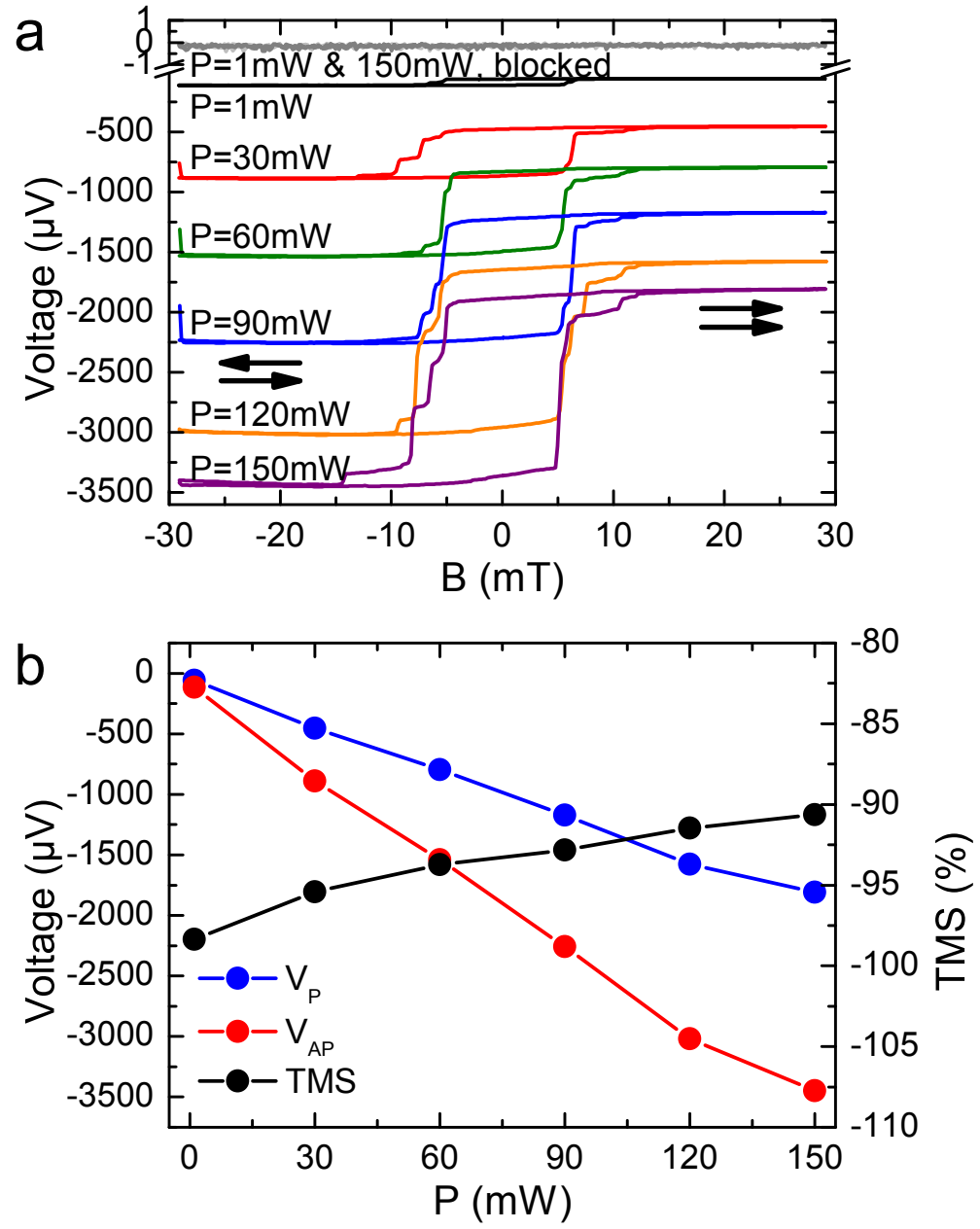

Figure 5.10: Laser power depedence of the TMS effect. a, TMS curves of a Heusler compound MTJ measured at different laser powers. The magnetization alignment is marked by the arrows. b, Thermovoltages (left scale) for parallel (blue circles) and antiparallel (red circles) magnetization alignment as a function of applied laser power. Additionally, the TMS values (right scale, black circles) are given for each laser power. 
The change in TMS ratio is small compared to Eggebrecht's results ${ }^{79}$. In that work, the TMS ratios are highest for low intensity and decrease with increasing intensity, in some cases the TMS ratio drops from $60 \%$ to $40 \%$. Moreover, this decrease is not always linear.

Furthermore, the tunnel magneto-Seebeck ratios observed in the $\mathrm{Co}-\mathrm{Fe}-\mathrm{B} / \mathrm{MgO}$ junctions were never of comparable size to the tunnel magnetoresistance ratio. But as can be seen in Fig. 5.10b and Fig. 5.8b, the TMR (102\%) and TMS (90\%-100\%) ratios are almost equal. Such an analogy has so far only been observed by Lin et $a l .{ }^{37}$ in alumina tunnel junctions.

\subsubsection{Thermocurrent measurements}

Thermocurrent measurements on $\mathrm{Co}-\mathrm{Fe}-\mathrm{B} / \mathrm{MgO}$ MTJs were already presented in section 3.4.2. It was observed that the thermocurrent in a closed circuit measurement also depends on the magnetization alignment of the electrodes. A MTJ that shows $3 \%$ TMS effect and a TMR ratio of $70 \%$ yielded a change of approximately $24 \%$ in the thermocurrent. The same behavior was reported by Liebing et al. ${ }^{41}$ for the same material system, but using resistive instead of optical heating. On the other hand, no magnetic thermocurrent effects were found in alumina based tunnel junctions, which were studied by Lin et $a l .{ }^{37}$, contrary to the findings in the $\mathrm{Co}-\mathrm{Fe}-\mathrm{B} / \mathrm{MgO}$ system.

For this reason, several thermocurrent measurements were carried out at different laser powers. The results are shown in Fig. 5.11. In Fig. 5.11a, a measurement at $90 \mathrm{~mW}$ laser power with magnified current axis is shown as an example. The typical minor loop of the MTJ is distinguishable. However, the effect ratio is less than $1 \%$. This is in sharp contrast to the observations made on Co-Fe-B/MgO MTJs in section 3.4.2, in which the thermocurrent effect ratio is even higher than the TMS ratio. Moreover, the measurements at different laser powers reveal that, on the scale of the power-dependent change in thermocurrent, no magnetic effects can be identified - in opposition to the TMS measurements shown in Fig. 5.10. These results are similar to the thermocurrent behavior of alumina based MTJs published by Lin et $a l .{ }^{37}$, who explain the absence of a magnetic effect in the thermocurrent with the properties of the electronic density of states in the $\mathrm{CoFe} / \mathrm{Al}_{2} \mathrm{O}_{3}$ system and a model based on the Jullière model.

\subsubsection{Position-dependent measurements}

To investigate the local generation of the Seebeck voltage, position-dependent measurements were performed, in which the laser spot was moved away from the contacted tunnel junction. Fig. 5.12a shows a sketch of the bond pad microstructure including the positions at which the measurements shown in Fig. 5.12b were made. The positions 1 to 4 are in the close vicinity of the MTJ, less than $30 \mu \mathrm{m}$ away 


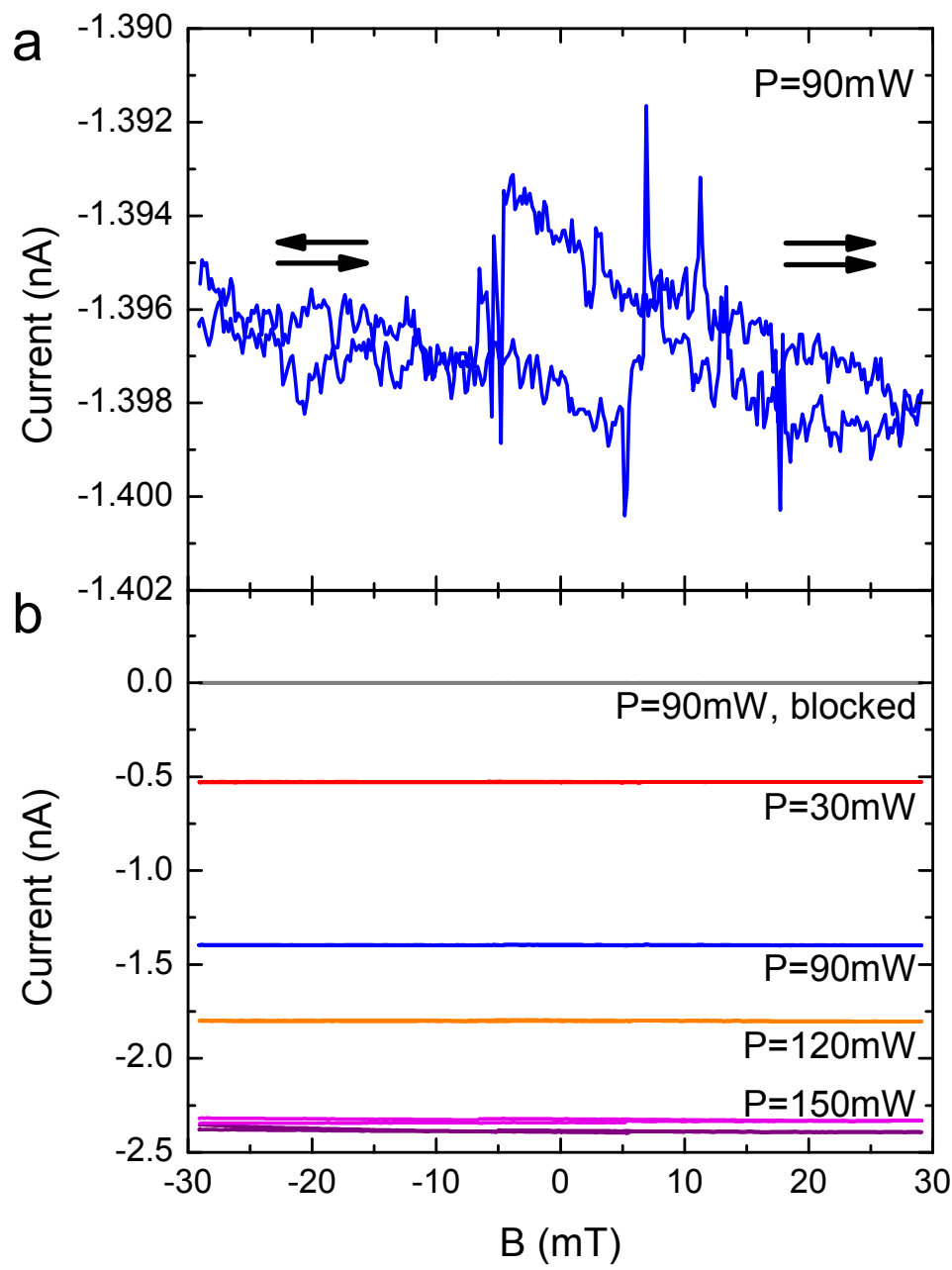

Figure 5.11: Thermocurrent measurements of Heusler compound MTJs. a, Thermocurrent curve measured at $90 \mathrm{~mW}$ laser power. A small magnetic effect of less than $1 \%$ is visible. $\mathbf{b}$, Thermocurrents measured at different laser powers. On this scale, no magnetic switching can be identified for any of the laser powers. 
a

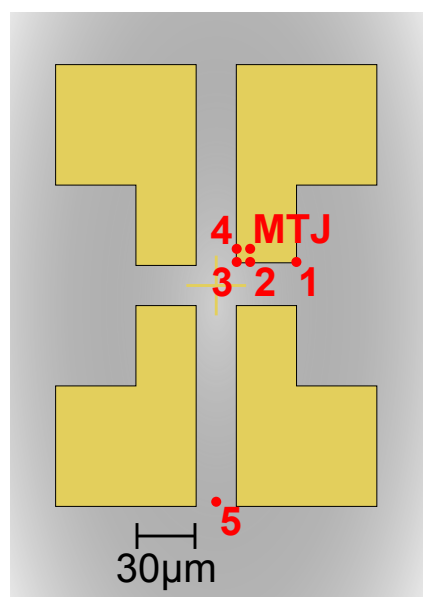

b

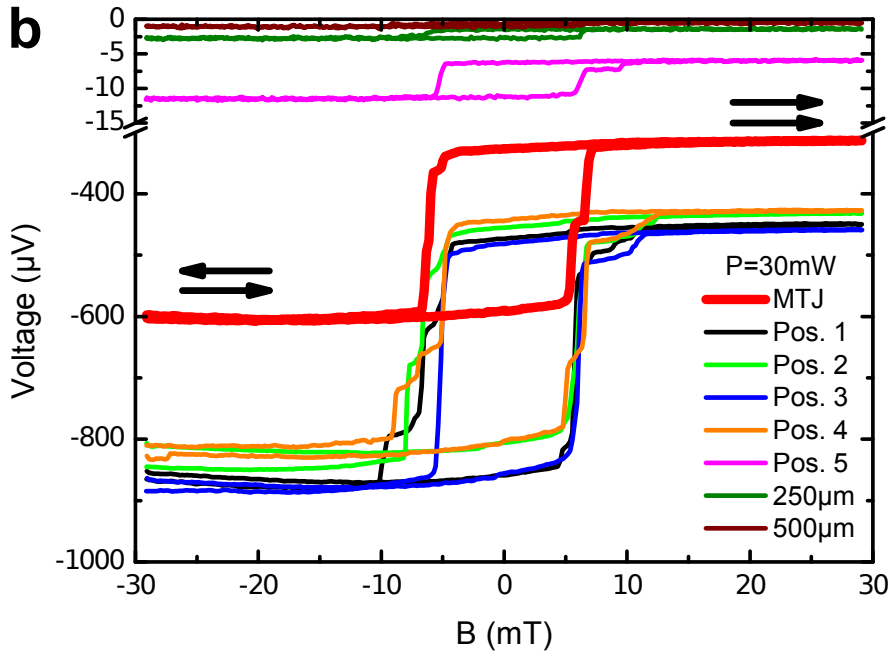

Figure 5.12: Position-dependent measurements of Heusler compound MTJs.

a, Sketch of the microstructured bond pads on the sample in which the positions of the laser spot are marked. b, TMS measurements of the upper right MTJ with the laser focussed on the different positions.

from the contacted MTJ, on the edges of the bond pad microstructure. Position 5 is located at the opposite end of the group of four bond pads, at a distance of approximately $130 \mu \mathrm{m}$ to the MTJ. In Fig. 5.12b two additional measurements, not displayed in Fig. 5.12a, are shown, in which heating was performed at distances of $250 \mu \mathrm{m}$ and $500 \mu \mathrm{m}$ away from the MTJ.

The Seebeck voltage that is generated when heating at positions 1 to 4 is even larger than when heating the MTJ directly, possesses the same sign and exhibits magnetic switching, as shown in Fig. 5.12b. However, this voltage only occurs when the laser spot is centered on the edge of the bond pad. It has to be noted that the positioning of the laser spot on the highly thermally conductive gold bond pad does not create a Seebeck voltage. In principle, an explanation for the voltage generation at the bond pad edges would be that one half of the laser spot creates a not radially symmetric, planar temperature gradient in the lower ferromagnetic layer extending to the MTJ, which causes a Seebeck voltage. In this case, a change of voltage sign would be expected, as observed by Eggebrecht, who investigated the positiondependence on comparable length scales, but found a sharp drop of the voltage and an inversed voltage sign when heating next to the bond pad and close to the $\mathrm{MTJ}^{79}$. This is opposed to the results shown in Fig. 5.12b. The position-dependent measurements on $\mathrm{Co}-\mathrm{Fe}-\mathrm{B} / \mathrm{MgO}$ tunnel junctions discussed in section 3.6 showed a small residual voltage of the same sign at comparable length scales (P2 in Fig. 3.6). However, due to different bond pad dimensions in that case, the edge of the bond pad was not reached, leading to a different scenario. 
At position 5, $130 \mu \mathrm{m}$ away from the MTJ, the voltage signal has already dropped by a factor of 100 and decreases further at $250 \mu \mathrm{m}$ and $500 \mu \mathrm{m}$. This indicates that the observed voltages are generated locally in the MTJ and compares to the position-dependence of $\mathrm{Co}-\mathrm{Fe}-\mathrm{B} / \mathrm{MgO}$ MTJs discussed in section 3.6. The curves in Fig. 5.12b show magnetic switching, even at a $250 \mu \mathrm{m}$ distance from the MTJ, whereas Eggebrechts' measurements on $\mathrm{Co}-\mathrm{Fe}-\mathrm{B} / \mathrm{MgO}$ tunnel junctions ${ }^{79}$ and those discussed in section 3.6 did not, apart from when the junction was directly heated by the laser. This magnetic effect might be caused by the difference in the patterning of the Heusler compound sample: To get a good crystalline quality of the $\mathrm{Co}_{2} \mathrm{FeSi}$ layer, it has to be grown on a crystalline $\mathrm{MgO}$ substrate with a thin chromium seed layer. The argon ion milling step for defining the MTJ pillar then removes the material down to the middle of the $\mathrm{Co}_{2} \mathrm{FeSi}$ layer. Thus, a ferromagnetic $\mathrm{Co}_{2} \mathrm{FeSi}$ layer remains that covers the whole sample and acts as a common bottom contact for all MTJs on the sample. A temperature gradient, created in this ferromagnetic layer by focussing the laser next to the bond pads, might lead to a magnetic effect in the Seebeck voltage. On the contrary, $\mathrm{Co}-\mathrm{Fe}-\mathrm{B} / \mathrm{MgO}$ tunnel junctions are grown on rather thick paramagnetic tantalum and/or ruthenium layers and the junction stack is etched down into the $\mathrm{Ta} / \mathrm{Ru}$ beneath the lower $\mathrm{Co}-\mathrm{Fe}-\mathrm{B}$ layer. Thus, no ferromagnet remains next to the bond pads that could create any magnetic effects in the Seebeck voltage.

\subsubsection{Discussion}

Although the first studies of MTJs employing Heusler compounds as electrodes yield interesting and promising results, further experiments are necessary to investigate some of the striking differences that these junctions show in comparison to the $\mathrm{Co}-\mathrm{Fe}-\mathrm{B} / \mathrm{MgO}$ system.

The Seebeck coefficients observed in $\mathrm{Co}-\mathrm{Fe}-\mathrm{B} / \mathrm{MgO}$ tunnel junctions are negative and agree well with theoretical predictions in their order of magnitude and their voltage sign. For $\mathrm{Co}_{2} \mathrm{FeSi} / \mathrm{MgO} / \mathrm{CoFe}$ tunnel junctions, no theoretical predictions exist so far. Ab initio calculations should be carried out in the future to allow a comparison of the huge thermovoltages observed and the positive Seebeck coefficients found in the experiment to theoretical expectations.

In section 5.2.4 it is found that only a very small magnetic effect can be observed in thermocurrent measurements. As discussed in that section, the results are similar to the observations by Lin et al. on $\mathrm{CoFe} / \mathrm{Al}_{2} \mathrm{O}_{3}$ tunnel junctions ${ }^{37}$. Moreover, the observation of almost equal TMR and TMS ratios are in accordance with that publication. These results are in contrast to observations made on Co$\mathrm{Fe}-\mathrm{B} / \mathrm{MgO}$ MTJs and contradict the expectation that in general the thermocurrent should show magnetic switching conforming to the thermodynamic kinetic equation (3.1), if TMR and TMS are existent. As a consequence, $\mathrm{CoFe} / \mathrm{Al}_{2} \mathrm{O}_{3}$ tunnel junctions and further Heusler compound MTJs (e.g. containing $\mathrm{Co}_{2} \mathrm{FeAl}$ ) should 
be investigated in prospective studies to extend the understanding how the specific (spin-dependent) electronic structure of these material systems leads to the discussed properties of the thermomagnetoelectric effects.

The position-dependent measurements presented in section 5.2.5 show a decrease of the Seebeck voltage with increasing distance of the laser spot from the MTJ. This indicates a local generation of the Seebeck voltage in the MTJ. However, a magnetic switching of the Seebeck voltage is observed even at larger distances. Further, voltage curves similar to those measured with direct heating of the MTJ are observed when the laser spot is placed on the bond pad edges whereas no voltage is created when the laser heats an area in the middle of the bond pad.

In further experiments these observations should be studied more closely. To verify that no parasitic voltages are created in the experiment, additional samples with different bond pad sizes and geometries should be fabricated to understand the voltage generation at the bond pad edges. The magnetic switching of the Seebeck voltage at larger distances between laser spot and MTJ could be due to other spin-dependent thermoelectric effects, such as e.g. the spin-Seebeck effect or the anomalous Nernst effect. The existence of these effects in Heusler compounds have recently been reported in the literature ${ }^{82}$. To determine if the magnetic effect observed in position-dependent measurements is caused by the $\mathrm{Co}_{2} \mathrm{FeSi}$ layer covering the whole sample as a common bottom contact for the MTJs, a set of samples should be prepared in which either the $\mathrm{Co}_{2} \mathrm{FeSi}$ layer is also patterned as a contact line, or the layer stack is etched down to the thin Cr seed layer. The high lead resistance caused by the thin chromium layer should be of no concern, at least for the measurements of the Seebeck voltage.

\subsection{The thermoelectric figure of merit}

In the previous chapters and sections, it has been shown that Seebeck voltages generated in magnetic tunnel junction can be switched magnetically into two voltage states. Further, the first results of section 5.1 suggest that the Seebeck voltages can be tuned in such a way that a thermoelectric device can be realized which can be switched on and off depending on the magnetization alignment of the two ferromagnets in the MTJ. Taking into account the feasibility of thermal spin-transfer torque (chapter 4), it might even be possible to switch the magnetization state thermally. But how efficient are magnetic tunnel junctions as thermoelectric devices? To answer this question, the thermoelectric figure of merit, which is connected to the efficiency of thermoelectric devices, is estimated in the following for the tunnel junctions presented in this thesis.

The thermoelectrical figure of merit can be derived by analyzing the heat flow in a Peltier element which is used either as cooling device or as a thermoelectric power generator. It can be shown ${ }^{20}$ that the maximum temperature achieved in a 
cooling device is

$$
\left(T_{\mathrm{h}}-T_{\mathrm{c}}\right)_{\max }=\frac{1}{2} Z T_{\mathrm{c}}^{2}
$$

and that the Carnot efficiency of the thermoelectric power generator can be expressed as

$$
\eta_{\max }=\frac{\left(T_{\mathrm{h}}-T_{\mathrm{c}}\right)\left(\sqrt{1+Z T_{\mathrm{av}}}+1\right)}{T_{\mathrm{h}}\left(\sqrt{1+Z T_{\mathrm{av}}}+\frac{T_{\mathrm{c}}}{T_{\mathrm{h}}}\right)},
$$

in which $T_{\mathrm{h}}, T_{\mathrm{c}}$ and $T_{\mathrm{av}}=\frac{T_{\mathrm{h}}+T_{\mathrm{c}}}{2}$ denote the hot, cold and average temperatures of the device, respectively. In these equations, the parameter $Z$ presents the socalled thermoelectric figure of merit which is dependent on the material's Seebeck coefficient $S$ and its electrical $(\sigma)$ as well as thermal conductivity $(\kappa)$ :

$$
Z=\frac{S^{2} \sigma}{\kappa}
$$

Since the unit of $Z$ is $\mathrm{K}^{-1}$, the dimensionless value $Z T$ is typically given in literature, where $T$ is the operating temperature of the device. Extensive research has been done in the last decades to create materials with a high $Z T$. At room temperature, the best alloy materials exhibit figures of merit of $Z T \approx 1$, whereas values as high as $Z T=2.5$ have been found in superlattices ${ }^{20}$.

For the following estimation of the figure of merit $Z T$, Seebeck coefficients reported for $\mathrm{Co}-\mathrm{Fe}-\mathrm{B} / \mathrm{MgO}$ junctions in the previous chapters will be taken into account, although $Z T$ can in principle be optimized by tuning the Seebeck coefficients, e.g. by choice of material. The thermal and electrical conductivities present, however, a possibility to alter the $Z T$ factor by several orders of magnitude just by changing layer thicknesses. The most important layers in the MTJ layer stack are the two ferromagnetic electrodes and the tunneling barrier, because these layers are responsible for the spin-dependent thermoelectric effect that is of interest.

The electrical conductivity in MTJs is dominated by the tunneling resistance of the barrier. For low voltages Simmons derived a theoretical model that describes the current density $I / A$ as function of the barrier thickness $t_{\mathrm{MgO}}$ (ref. [83]):

$$
\frac{I}{A}=\frac{V}{t_{\mathrm{MgO}}} \frac{3 \sqrt{2 m_{e} \varphi}}{2}\left(\frac{e}{h}\right)^{2} \exp \left(\frac{4 \pi t_{\mathrm{MgO}}}{h} \sqrt{2 m_{e} \varphi}\right)
$$

in which $m_{e}$ is the electron mass, $h$ Planck's constant and $\varphi$ the height of the potential barrier. The electrical conductivity is obtained by multiplying this equation by $t_{\mathrm{MgO}} / V$ and inserting numerical values for the constants in suitable units:

$$
\sigma=3.16 \cdot 10^{4} \sqrt{\varphi} \exp \left(-1.025 \sqrt{\varphi} t_{\mathrm{MgO}}\right)
$$

in which $t_{\mathrm{MgO}}$ is in $\AA$ and $\varphi$ in volt. The unit of the electrical conductivity is $\Omega^{-1} \mathrm{~m}^{-1}$. 

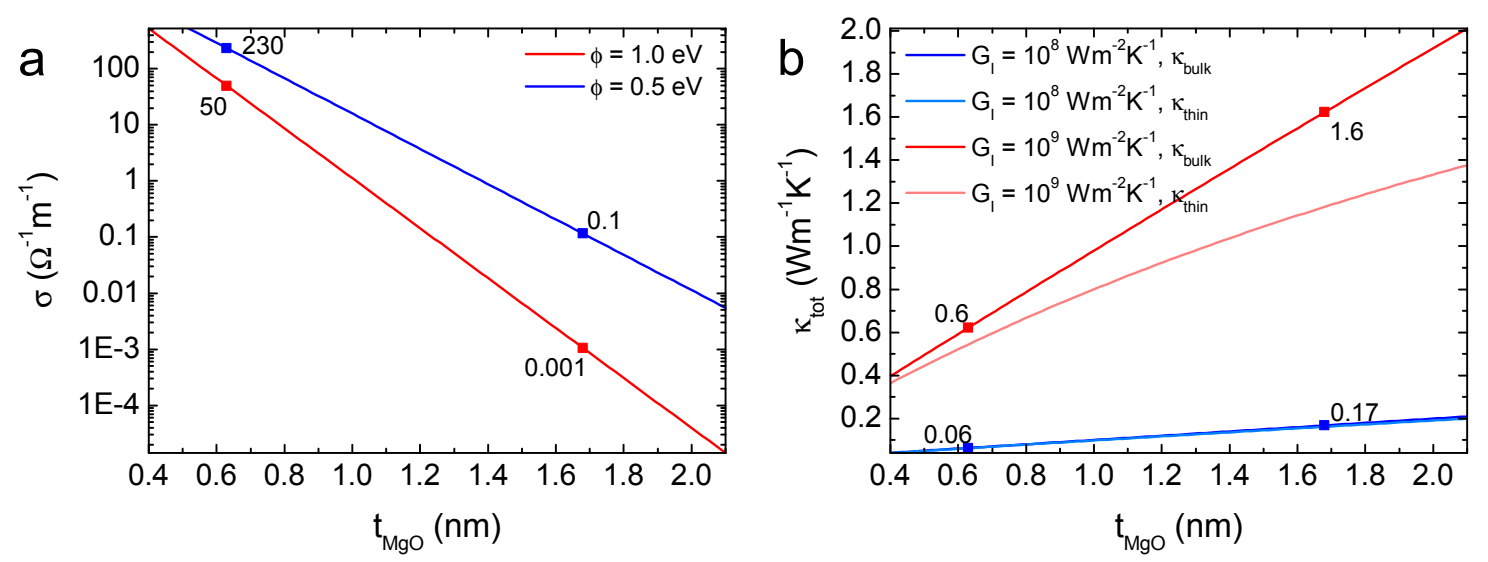

Figure 5.13: Electrical and thermal conductivities of MTJs as function of barrier thickness. a, Electrical conductivity of the MTJ (eq. (5.8)) for barrier heights of $0.5 \mathrm{eV}$ and $1.0 \mathrm{eV}$. Values are given for 3 and 8 monolayers of $\mathrm{MgO}$. b, Thermal conductivity of the $\mathrm{MgO}$ barrier plus its interfaces. Assumed are interface conductances of $1 \times 10^{8} \mathrm{~W} \mathrm{~m}^{-2} \mathrm{~K}^{-1}$ and $1 \times 10^{9} \mathrm{~W} \mathrm{~m}^{-2} \mathrm{~K}^{-1}$. Further, the thermal conductivity of the $\mathrm{MgO}$ layer is either taken as $k_{\text {thin }}=4 \mathrm{~W} \mathrm{~m}^{-1} \mathrm{~K}^{-1}$ or as $k_{\text {bulk }}=$ $48 \mathrm{~W} \mathrm{~m}^{-1} \mathrm{~K}^{-1}$. Again, values for 3 and 8 monolayers of $\mathrm{MgO}$ are given.

Fig. 5.13a shows the thickness dependence according to eq. (5.8) for two barrier heights that are typically found for $\mathrm{Co}-\mathrm{Fe}-\mathrm{B} / \mathrm{MgO} \mathrm{MTJ}^{84}$. With increasing barrier thickness, the electrical conductivity decreases exponentially and the slope is determined by the barrier height. The thinnest barrier that could be fabricated experimentally is 3 monolayers of $\mathrm{MgO}(0.63 \mathrm{~nm})$, whereas in most cases $\mathrm{MgO}$ thicknesses of 8 monolayers are used for the thermoelectric measurements. Fig. 5.13a shows that, depending on the assumed barrier height, the electrical conductivity can be enhanced by a factor of $10^{3}$ to $10^{4}$ through reduction of the $\mathrm{MgO}$ barrier thickness from 8 monolayers to 3 monolayers.

The thermal conductivity is estimated by taking into account the thermal conductivity of the $\mathrm{MgO}$ layer and its two interfaces to the adjacent Co-Fe-B layers. This configuration presents a series of thermal resistances such that the total resistance (written in terms of conductances) yields

$$
\frac{1}{G_{\mathrm{tot}}}=\frac{2}{G_{I}}+\frac{t_{\mathrm{MgO}}}{\kappa_{\mathrm{MgO}}} \Leftrightarrow G_{\mathrm{tot}}=\frac{G_{I} \kappa_{\mathrm{MgO}}}{2 \kappa_{\mathrm{MgO}}+G_{I} t_{\mathrm{MgO}}},
$$

with the interface conductance $G_{I}$ and the thermal conductivity of $\mathrm{MgO} \kappa_{\mathrm{MgO}}$. The thermal conductivity of the $\mathrm{MgO}$ layer plus interfaces, $\kappa_{\mathrm{tot}}$, is obtained by multiplying the conductance with the $\mathrm{MgO}$ thickness:

$$
\kappa_{\mathrm{tot}}=G_{\mathrm{tot}} t_{\mathrm{MgO}}=\frac{G_{I} \kappa_{\mathrm{MgO}} t_{\mathrm{MgO}}}{2 \kappa_{\mathrm{MgO}}+G_{I} t_{\mathrm{MgO}}} .
$$


The total thermal conductivity of the $\mathrm{MgO}$ barrier including its two interfaces according to eq. (5.9) is plotted in Fig. 5.13b for different assumptions of $G_{I}$ and $\kappa_{\mathrm{MgO}}$. It is reported in literature that the interface conductance $G_{I}$ depends only weakly on the type of interface ${ }^{85}$ and is of the order of $1 \times 10^{8} \mathrm{~W} \mathrm{~m}^{-2} \mathrm{~K}^{-1}$ to $1 \times 10^{9} \mathrm{~W} \mathrm{~m}^{-2} \mathrm{~K}^{-1}$ (ref. [20]). The total thermal conductivity of the $\mathrm{MgO}$ layer is depicted in Fig. 5.13b for both interface conductances. In both cases, a reduction by more than $50 \%$ of the thermal conductivity is found when the $\mathrm{MgO}$ barrier thickness is reduced from 8 monolayers to 3 monolayers. Further, a thin film value $\left(\kappa_{\text {thin }}=4 \mathrm{~W} \mathrm{~m}^{-1} \mathrm{~K}^{-1}\right)$ and a bulk value $\left(\kappa_{\text {bulk }}=48 \mathrm{~W} \mathrm{~m}^{-1} \mathrm{~K}^{-1}\right)$ are assumed for the thermal conductivity of the $\mathrm{MgO}$ layer itself. But since the interface conductances dominate the total thermal conductivity, especially for $G_{I}=1 \times 10^{8} \mathrm{~W} \mathrm{~m}^{-2} \mathrm{~K}^{-1}$, the choice of the layer thermal conductivity has only a small effect.

As shown in Fig. 5.13, the thermoelectric figure of merit can be strongly enhanced by reducing the $\mathrm{MgO}$ barrier thickness because of the resulting increase of electrical and decrease of thermal conductivity. The thermoelectric figure of merit $Z T$ as function of barrier thickness is presented in Fig. 5.14. It is calculated according to eq. (5.7), multiplied by $T=300 \mathrm{~K}$ and using eqs. (5.8) and (5.9), as well as a $\mathrm{MgO}$ layer thermal conductivity of $\kappa_{\text {bulk }}=48 \mathrm{~W} \mathrm{~m}^{-1} \mathrm{~K}^{-1}$. Several magnitudes

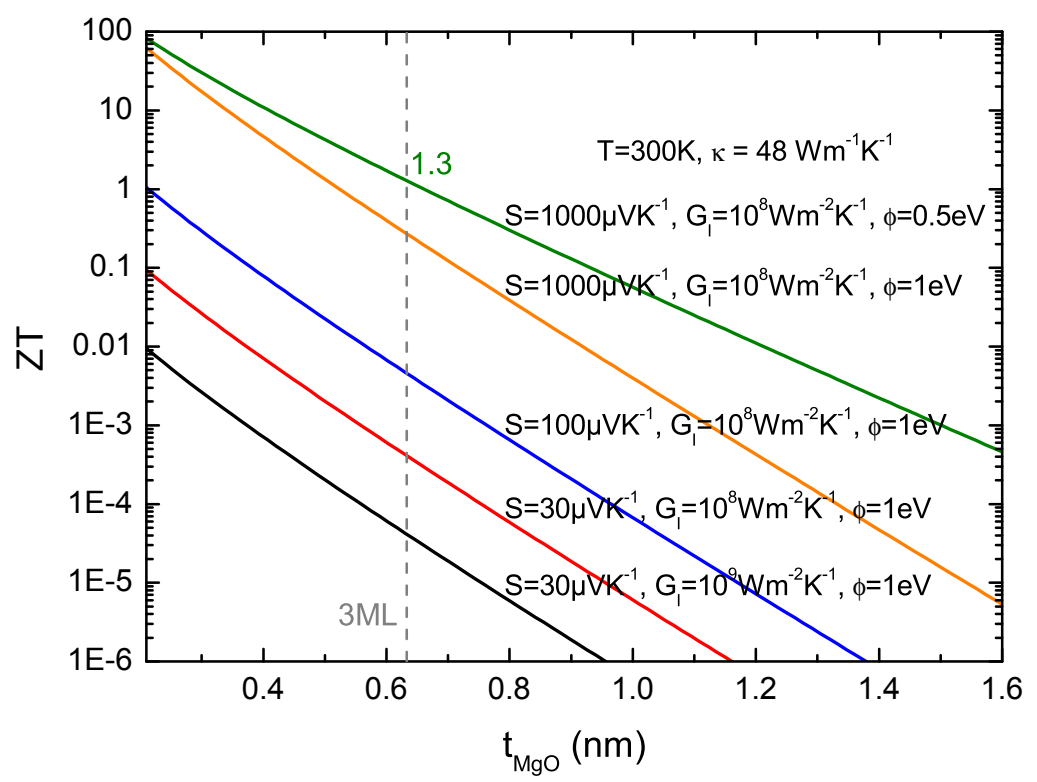

Figure 5.14: Thermoelectric figure of merit of Co-Fe-B/MgO MTJs. The thermoelectric figure of merit $Z T$ is calculated according to eq. (5.7) (multiplied by $T=300 \mathrm{~K}$ ) for different magnitudes of the parameters entering this equation. A thermal conductivity of $48 \mathrm{~W} \mathrm{~m}^{-1} \mathrm{~K}^{-1}$ is assumed for the $\mathrm{MgO}$ barrier. In addition, the experimental thin film limit of 3 monolayers $\mathrm{MgO}$ is marked by the gray dashed line. 
are assumed for the physical quantities contributing to $Z T$ in eq. (5.7). First, a Seebeck coefficient of $30 \mathrm{\mu VK}^{-1}$ is taken (see also section 5.1.3), combined with an interface conductance of $1 \times 10^{9} \mathrm{~W} \mathrm{~m}^{-2} \mathrm{~K}^{-1}$ and a barrier height of $\varphi=1.0 \mathrm{eV}$ (black line in Fig. 5.14). A first order of magnitude enhancement of $Z T$ is achieved by changing the interface conductance to $1 \times 10^{8} \mathrm{~W} \mathrm{~m}^{-2} \mathrm{~K}^{-1}$ (red line in Fig. 5.14). According to literature ${ }^{20,85}$, interface conductances of $1 \times 10^{9} \mathrm{~W} \mathrm{~m}^{-2} \mathrm{~K}^{-1}$ are realistic only for perfect interfaces. As discussed in chapter 4 , the crystalline quality and thus also the quality of the interfaces is lower for 3 monolayer $\mathrm{MgO}$ barriers. Consequently, the assumption of $G_{I}=1 \times 10^{8} \mathrm{~W} \mathrm{~m}^{-2} \mathrm{~K}^{-1}$ can be justified, especially in the 3 monolayer limit. An improvement in $Z T$ of another magnitude is obtained by assuming a Seebeck coefficient of $100 \mu \mathrm{VK}^{-1}$ (blue line in Fig. 5.14). This value is still realistic, both from an experimental (chapter 2) and theoretical (chapter 2 and section 5.1) point of view. Even Seebeck coefficients in the range of $1000 \mu \mathrm{V} \mathrm{K}^{-1}$ might be realizable (chapter 3 ). However, these values were obtained by using temperature simulations that indeed considered the reduced value of the thermal conductivity in the thin $\mathrm{MgO}$ barrier, but did not take thermal resistances of interfaces into account. Hence, the results can be regarded as an upper limit of the Seebeck coefficient. On the other hand, a Seebeck coefficient of $1000 \mu \mathrm{V} \mathrm{K}{ }^{-1}$ already leads to a $Z T$ close to 1, comparable with today's best thermoelectric alloys $^{20}$. The barrier height of $\varphi=1 \mathrm{eV}$ was found ${ }^{84}$ for MTJs showing a TMR ratio of approximately $100 \%$. In high quality MTJs with TMR ratios larger than $200 \%$, barrier heights of $\varphi=0.5 \mathrm{eV}$ are also possible. A reduced barrier height leads to a less steep decrease of $Z T$ with increasing $\mathrm{MgO}$ thickness and further improves the thermoelectric figure of merit (green line in Fig. 5.14). At the 3 monolayer limit, even a $Z T$ of 1.3 is achieved.

It can be concluded from these estimations that magnetic tunnel junctions have the potential to be efficient thermoelectric devices. Assumptions that are feasible experimentally already lead to $Z T=1.3$. The Seebeck coefficient of the MTJ that contributes to $Z T$ quadratically might be even further improved by materials engineering of the ferromagnetic leads. One might also think of using double barrier junctions with very thin $\mathrm{MgO}$ barriers to introduce additional interfaces which decrease the thermal conductivity and at the same time lead to higher temperature gradients in those layers that are important for the tunnel magneto-Seebeck effect. Another option to decrease the thermal conductivity of the MTJ as a whole would be to use nanolaminates ${ }^{86}$ as electrical lead layers. This, however, will improve the thermoelectric figure of merit, but then reduce the tunnel magneto-Seebeck effect, because of the increased spin-independent Seebeck voltage created in these layers. 



\section{Chapter 6}

\section{Summary}

The demand for higher computational powers and higher storage capacities has led to an on-going miniaturization process of logic and storage devices, while maintaining and even optimizing their computational speed. As a consequence, the power consumption of these devices is steadily increasing and a physical limit of this process is conceivable, in which the power dissipation is no longer tolerable. Thus, new concepts and ideas are sought-after to overcome this limit.

Solid-state devices that utilize the spin of an electron as well as its charge, i.e. spintronic devices, offer interesting possibilities in this regard. For this reason, the research field of spin caloritronics has emerged in the last decade, which is committed to the investigation of the spin degree of freedom in the thermoelectric properties of spintronic devices. This field has attracted considerable attention in recent years, and the experimental as well as theoretical work presented in the introductory chapter 1 is only a small, albeit important, part of the progress that has been made by the scientific community.

The present thesis contributes to this exciting field with investigations of spindependent thermoelectric effects in magnetic tunnel junctions. The publication presented in chapter 2 provides the first experimental observation of the tunnel magneto-Seebeck effect in tunnel junctions, utilizing laser heating to generate the required temperature gradients. Finite-element simulations are employed to estimate the temperature distribution in the micro-structured device containing the MTJ. The observed Seebeck voltages and estimated Seebeck coefficients are in good agreement with $a b$ initio calculations, when a mixed Co-Fe interface is assumed to be the experimentally most realistic scenario. The calculations presented in this chapter shed first light on the strong influence of material parameters on the tunnel magneto-Seebeck effect.

Chapter 3 is devoted to the detailed investigation of the time-dependent Seebeck voltages down to the microsecond regime. The improvement of the experimental setup allows for the measurement of changes in the Seebeck voltage in the nanovolt regime and on the microsecond timescale. MTJs grown on $\mathrm{MgO}$ and thermally oxidized Si substrates show very similar Seebeck voltages and tunnel magneto-Seebeck ratios. Sharp peaks in the time-dependent voltage signals that were observed in 
chapter 2 do not appear in MTJs grown on $\mathrm{MgO}$ substrate. A model circuit of the MTJ is developed that is able to explain the voltage signals observed experimentally: The sharp peaks in the Seebeck voltage originate from Seebeck voltages induced in the Si substrate that couple capacitively through the thermally grown oxide of the substrate. Since no Seebeck voltages can be induced in an MgO substrate, these peaks do not appear in the time-dependent Seebeck voltage of MTJs grown on this substrate. Thermocurrent measurements are performed that also show a magnetic effect. In further experiments, the heating laser spot is moved away from the connected MTJ and a strongly decreasing Seebeck voltage is detected. These experiments verify that the Seebeck voltage is generated locally in the MTJ. Thus, a deeper insight into the tunnel magneto-Seebeck effect is gained in this chapter. The results of chapter 2 and 3 are furthermore in agreement with the resistive heating experiments on $\mathrm{Co}-\mathrm{Fe}-\mathrm{B} / \mathrm{MgO}$ tunnel junctions carried out by Liebing et al. ${ }^{39-41}$.

Chapter 4 deals with the experimental feasibility of the theoretically predicted thermal spin-transfer torque switching by investigating the parameter space, determined by Jia et $a l .{ }^{44}$. A quantitative HR-TEM analysis allows the optimization of the $\mathrm{MgO}$ growth conditions in terms of deposition temperature. It is shown that $\mathrm{Co}-\mathrm{Fe}-\mathrm{B} / \mathrm{MgO}$ tunnel junctions with a barrier thickness of only 3 monolayers $\mathrm{MgO}$ can be fabricated. Nanometer-sized junctions with these ultra-thin barriers, incorporating Co-Fe-B layers with perpendicular magnetic anisotropy, show remarkably low critical switching currents of less than $0.2 \mathrm{MA} \mathrm{cm}^{-2}$ in DC I-V-characteristics. The required temperature gradients in the order of $10 \mathrm{~K}$, as proposed by Jia et al., can be achieved by heating with a femtosecond laser, according to finite-element simulations. However, additional ab initio calculations predict that the thermal spin-transfer torque strongly depends on the Fermi level position and, hence, on the Co-Fe composition. The thermal torque can be orders of magnitude lower than predicted by Jia et al.

The importance of material parameters on the tunnel magneto-Seebeck effect has already been pointed out. The outlook in chapter 5 presents first results of the most recent experiments in which this topic is treated. The tunnel magnetoSeebeck effect is either tuned by applying an external voltage and thus shifting the electronic density of states of one ferromagnetic layer, or by utilizing a Heusler compound as a ferromagnetic electrode. The first results are already promising, but challenges remain for further theses, as discussed in the respective sections. In the last section of chapter 5, the thermoelectric figure of merit is estimated for magnetic tunnel junctions and it is suggested that MTJs have the potential to be efficient thermoelectric devices.

Although much progress has been made, several theoretically predicted effects, such as thermal spin-transfer torque switching, have not yet been observed in experiments. In addition to appropriate material systems (e.g. systems having a large Seebeck coefficient and showing a huge tunnel magneto-Seebeck effect), a deeper 
understanding of heat transport in nano-structured multilayers is necessary to optimize the thermal design of future spin caloritronic devices, in which considerable temperature gradients are generated only in the functional layers. Therefore, spin caloritronics and the tunnel magneto-Seebeck effect in tunnel junctions remain an exciting topic. Whether spintronic devices, which utilize waste heat for logic operations and consume small amounts of power, are the future devices of a greener information technology is a question that only further fundamental research can answer. 



\section{Appendix A}

\section{Supplementary information of Nature Materials 10, 742 (2011)}

\section{A.1 Details of the laser heating and Seebeck voltage transients}

The slowest time constant in the heating process arises from the low conducting electric isolation $\mathrm{SiO}_{2}$ layer on the $\mathrm{Si}$ wafer, which is the thermal bottleneck. The temperature gradient at the barrier itself, which is responsible for the voltage generation, is $4.4 \mathrm{mK}$, in accordance with the simple estimation using the ideal bulk value for $\mathrm{MgO}$ for the thermal conductivity $\kappa$. A closer fit of theoretical and experimental results will be possible with a more precise experimental determination of the temperature gradient at the $\mathrm{MgO}$ barrier, since it depends sensitively on the thermal conductivity of the thin films and interfaces and thus has the largest error bar. While, in principle, this task is possible for such thin films ${ }^{87}$, it will be difficult to accomplish, especially in a single device. However, the thin film value for the $2.1 \mathrm{~nm} \mathrm{MgO}$ layer is expected to be less than its bulk value of $\kappa=48 \mathrm{~W} /(\mathrm{m} \cdot \mathrm{K})$. For polycrystalline $\mathrm{MgO}$ films with a grain size of $3-7 \mathrm{~nm}$, a value of $\kappa=4 \mathrm{~W} /(\mathrm{m} \cdot \mathrm{K})$ was found at room temperature, which differs by a factor of ten ${ }^{47}$. This difference arises from the grain boundaries. In addition, the thermal resistance at the Co$\mathrm{Fe} / \mathrm{MgO}$ interface can be considerable, i.e., $1 \times 10^{9} \mathrm{~W} /\left(\mathrm{m}^{2} \cdot \mathrm{K}\right)$ [87]. This interface term is approximately of the same order as the thermal resistance of a film that is $1 \mathrm{~nm}$ thick and may further increase the thermal gradient and thus decrease the Seebeck coefficients calculated. Taking into account the crystalline quality of the tunnel junctions grown by solid-state epitaxy as derived from the high resolution TEM, the most realistic scenario is a model assuming bulk-like thermal conductivity for the $\mathrm{MgO}$ because of its crystalline quality and a thermal interface resistance at both $\mathrm{Co}-\mathrm{Fe} / \mathrm{MgO}$ interfaces. Thus, the value for the $\mathrm{MgO}$ tunnel barrier is expected to be between the thin film value for the polycrystalline material and the bulk value. Here we give the polycrystalline and the bulk value as a lower (conservative estimation) and upper limit (optimistic estimation).

In the following, we discuss the dynamic time scales that contribute to the voltage response to the laser heating experiments shown for different modulation frequencies 
Table A.1: For $\mathrm{MgO}$ the thin film value (bulk value) of the thermal conductivity given respectively.

\begin{tabular}{cccc}
\hline \hline Material & $\kappa(\mathrm{W} /(\mathbf{m} \cdot \mathbf{K}))$ & $\boldsymbol{\rho}\left(\mathbf{1 0}^{\mathbf{3}} \mathrm{kg} / \mathbf{m}^{\mathbf{3}}\right)$ & $\boldsymbol{c}_{\boldsymbol{V}} / \boldsymbol{c}_{\boldsymbol{p}}(\mathbf{J} / \mathbf{\mathrm { kg } \cdot \mathrm { K } ) )}$ \\
\hline $\mathrm{Ta}$ & 57 & 16.7 & 140 \\
$\mathrm{Ru}$ & 117 & 12.4 & 238 \\
$\mathrm{Au}$ & 320 & 19.3 & 128 \\
$\mathrm{Cr}$ & 94 & 7.2 & 449 \\
$\mathrm{MgO}$ & $4(48)$ & 3.6 & 935 \\
$\mathrm{SiO}_{2}$ & 1.4 & 2.2 & 1052 \\
$\mathrm{Co}_{20} \mathrm{Fe}_{60} \mathrm{~B}_{20}$ & 87 & 8.2 & 440 \\
\hline \hline
\end{tabular}

in Fig. 2.4a (main manuscript). Time traces have been measured after amplification (100-MHz PM 3382 Phillips oscilloscope). A negative voltage was found for the opening of the shutter upon heating. When the shutter is closed, the cooling reverses the sign of the voltage. The heat is effectively conducted away from the $17.5 \mu \mathrm{m}$ heat spot into the large $100 \mathrm{~nm}$ thick $\mathrm{Au}$ bond pads. The time constant of the voltage increases within a few $\mu$ s, i.e., the response of the voltages are approximately 10-100 us, generally asymmetric for the heating and cooling process. The dynamic response depends on three factors, all of which were in the microsecond regime, i.e., 1) the time constant of heating, 2) the width of the Gaussian laser intensity profile that moves across the chopper blade, and 3) the response time of the LT1113 precision operational amplifier to realize a high input impedance of $100 \mathrm{G} \Omega$. While the thermal equilibration time in the COMSOL model is $2 \mu \mathrm{s}$, and the response time of the amplifier $5 \mathrm{MHz}$, we identified the width of the Gaussian laser intensity profile that moves across the chopper blade as slowest contribution limiting the response time. The laser diameter and chopper blade dimensions (different for each modulation frequency with varied chopper blades) range from 20-150 $\mathrm{us}$ and can be extracted from the reference diode signal in Fig. 2.4a (main manuscript).

In addition, we conducted tests to determine whether spurious magneto voltages were generated in the layer stack. A natural failure of the barrier (dielectric breakdown) at low voltages allows shorten the junction without too much destruction of the element, and the magneto-Seebeck effect should vanish. To prove this claim, we conducted tests to determine whether magneto-Seebeck voltage was generated at the tunnel barrier. The tests consisted of gradually shorting the barrier, as evidenced by a decrease in the resistance from the $100 \mathrm{k} \Omega$ of an intact junction to $1 \mathrm{k} \Omega$ and, finally, to $100 \Omega$ for the shortened junction. In the intermediate state, the magneto-Seebeck effect decreased to about $1 \%$, but the magnetic effect in the 
Seebeck voltage vanishes completely if the $\mathrm{MgO}$ tunnel barrier is bridged. This excludes the existence of magneto voltages not generated at the tunnel barrier. However, a background voltage is still contributing to the signal, thereby limiting the experimentally determined value of the magneto-Seebeck effect, $S_{\mathrm{MS}}$. Also, we can exclude the generation of lateral spin currents. This was checked by reversing the direction of rotation of the chopper blade and thereby changing the direction of the possible lateral heat gradients in plane. This sign change should in that case also lead to sign reversal, which is not observed.
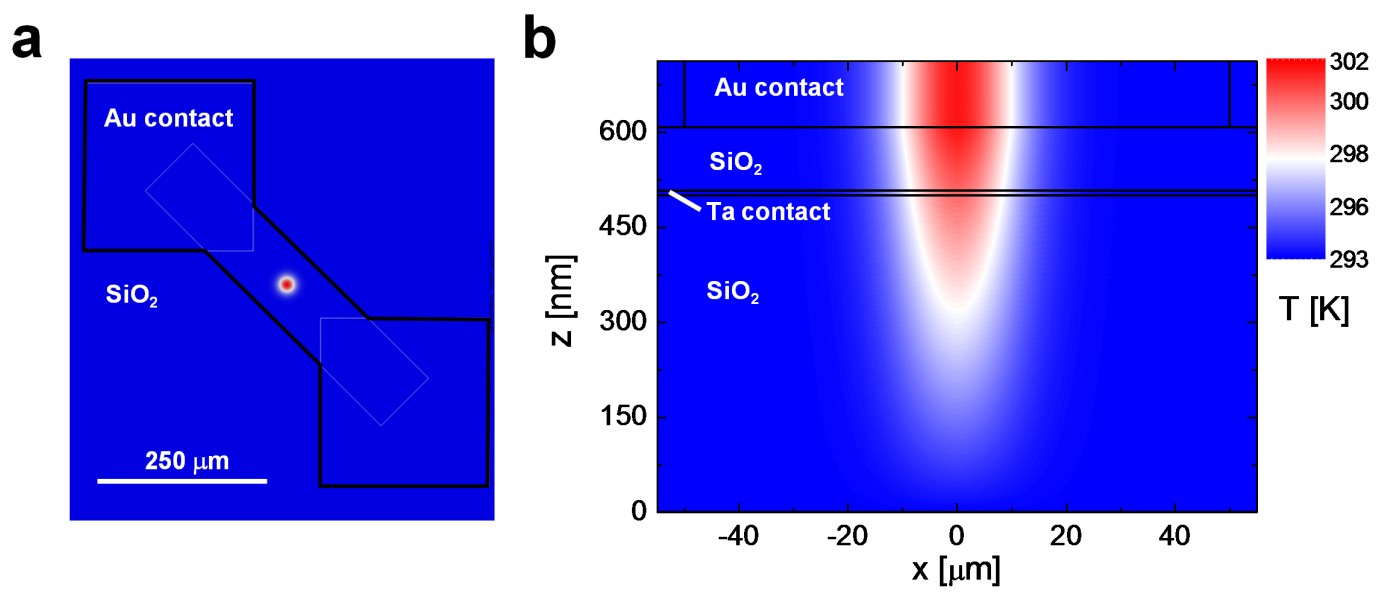

Figure A.1: COMSOL simulation on the larger length scale to illustrate the extension of the heating spot of $15 \mu \mathrm{m}$. The diameter of the spot heated up extends to $17.5 \mu \mathrm{m}$.

\section{A.2 Lateral heating in the Au bond pad}

Shown in Fig. A.1 is the full bond pad that has been simulated to reveal the extension of the heated area. The simulation shows that the Au bond pad is not heated homogeneously. The Gaussian beam diameter of $15 \mu \mathrm{m}$ results in a heated area of $17.5 \mu \mathrm{m}$ in diameter, not much further extended than the area heated by the laser spot. The reason is the large heat conductivity of $320 \mathrm{~W} /(\mathrm{m} \cdot \mathrm{K})$ of the $100 \mathrm{~nm}$ thick Au bond pad layer. During one modulation cycle first, the laser power is turned on the temperature rises. Second, when turned off, the large Au bond pads that have the highest heat conductivity work as a cooler. The temperature gradient is reversed in the junction. This is detected in the sign reversal of the Seebeck voltage for the transient voltage traces. 

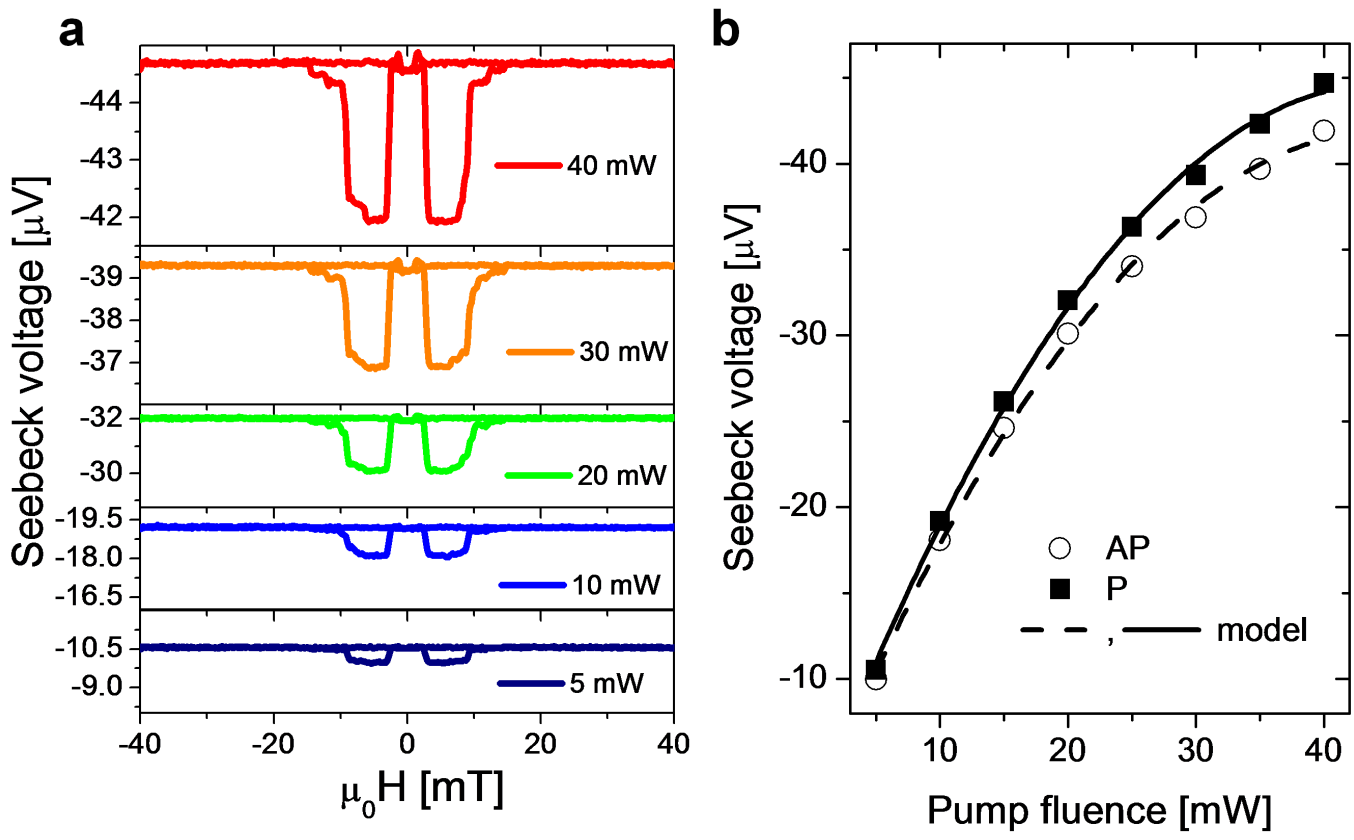

Figure A.2: Seebeck voltage measured for a tunnel junction heated with low laser fluencies (diode laser). a, Seebeck voltage versus applied magnetic field. b, Seebeck voltages for parallel and antiparallel orientation. The model is derived assuming a change of the Seebeck coefficient with temperature.

\section{A.3 Fluence dependence of the Seebeck voltages}

In the following the fluence dependence of the Seebeck voltage is discussed. The magnetoresistance of the junction is $120 \%$ and it shows a magneto-Seebeck effect of $6.2 \%$, which is somewhat smaller than for the element discussed in the main manuscript in particular. The voltage change from parallel to antiparallel configuration is $2.5 \mu \mathrm{V}$ (for $30 \mathrm{~mW}$ laser power). In the experiment the laser fluence is varied from 5 to $40 \mathrm{~mW}$. It can be seen that with increasing the laser fluence the shape of the signal does not change. However the Seebeck voltage increases as expected. For the highest laser fluence one finds $-45 \mu \mathrm{V}$ in the parallel configuration and $-42 \mu \mathrm{V}$ for the antiparallel configuration. To discuss the evolution in detail, the values are given in Fig. A.2. One observes that the voltage increases nonlinearly with the pump fluence. However this behavior is expected: with increasing the pump-fluence not only the temperature difference at the barrier increases, also the base temperature increases. One can calculate the voltage dependence from the following model

$$
V_{\mathrm{P}, \mathrm{AP}}=S_{\mathrm{P}, \mathrm{AP}} \Delta T=s_{\mathrm{P}, \mathrm{AP}} T a(T-293 \mathrm{~K})
$$


Here $s_{\mathrm{P}, \mathrm{AP}}$ is defined as the slope of the $S_{\mathrm{P}, \mathrm{AP}}$ versus $T$ curve. The parameter $a$ is given by the total junction geometry. It relates the temperature gradient at the junction $(\Delta T)$ to the temperature gradient in between the tunnel barrier $(T)$ and room temperature $(293 \mathrm{~K})$ at the substrate bottom. From a series of simulations using laser fluences from 5 to $40 \mathrm{~mW}$, we estimate a value of $1.8 \mathrm{mK} / \mathrm{mW}$ as rise in the temperature gradient $\Delta T$ with laser power. The increase of the temperature at the tunnel barrier is $1.2 \mathrm{~K} / \mathrm{mW}$ with laser power. In addition, the theoretical model predicts that the Seebeck coefficient itself decreases as the base temperature is raised. Assuming a constant slope $s_{\mathrm{P}, \mathrm{AP}}$, we can qualitatively understand the linear plus quadratic behavior of the data in Fig. A.2b.
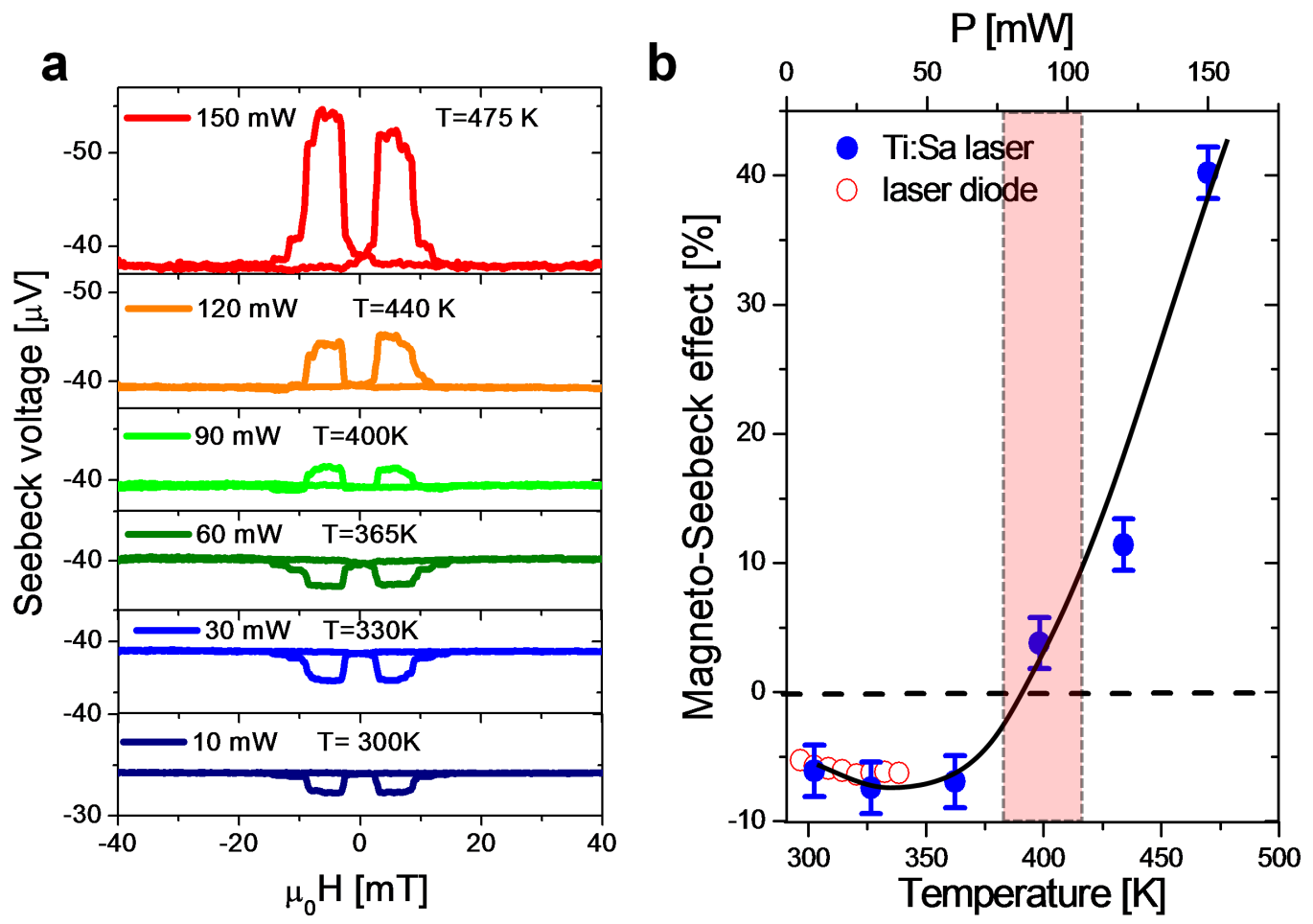

Figure A.3: Seebeck voltage measured for a tunnel junction heated with high laser fluences (Ti:sapphire oscillator, central wave length $\lambda=810 \mathrm{~nm}$ ). a, Seebeck voltage versus applied magnetic field. b, Magneto-Seebeck effect as a function of the laser power and corresponding temperature calculated by the numerical model. The data for the diode laser is shown for direct comparison. A sign change is observed in the region at around $400 \mathrm{~K}$ as expected from the theoretical model.

Even larger fluences are reached using a Ti:sapphire laser, which can be regarded here as a cw laser source at moment. This is justified to a good approximation because of the large distance from the Au top layer to the tunnel barrier $(>130 \mathrm{~nm})$. We find that the magneto-Seebeck values for high laser fluences lie on top of the 
previous data determined using the cw diode laser, both shown in the shown in the same figure for direct comparison (Fig. A.3b). One observes a characteristic sign change of the magneto-Seebeck effect in-between 60 and $90 \mathrm{~mW}$. This sign change is predicted by our theoretical model (Fig. 2.1e, main manuscript). By relating the pump fluence to a temperature using the numerical finite element simulation, one can plot the magneto-Seebeck effect as a function of the temperature derived at the tunnel junction (Fig. A.3b). For the largest laser power the base temperature can be increased up to $475 \mathrm{~K}$. The sign reversal takes place at around the predicted compensation point for the Seebeck voltages for parallel and antiparallel orientation at $400 \mathrm{~K}$. However there are also features observed not expected from the model. The magneto-Seebeck effect shows a decrease in between 300 to $350 \mathrm{~K}$ before it rises and crosses zero. However, the magneto-Seebeck effect reaches $40 \%$ for $150 \mathrm{~mW}$ laser power, close to the theoretically expected value. The maximum voltage change from parallel to antiparallel configuration is $15 \mu \mathrm{V}$. Altogether, the finding of the prominent feature, the sign reversal with increased temperature, supports the conclusion that the transport coefficients calculated according to the model of coherent tunneling can explain characteristic features and thus is expected to be the dominating contribution to the magneto-Seebeck effect.

In the high fluence range one may ask if for such large temperature gradients the theoretical model is still valid. The temperature gradient at the $2.1 \mathrm{~nm}$ thick $\mathrm{MgO}$ barrier is $270 \mathrm{mK}$ for the highest fluence. To answer this question, it is helpful to compare this temperature to an energy scale. Bias dependence is calculated in such a system for up to $1 \mathrm{~V}$ bias voltage ${ }^{88}$. This amounts to $1 \mathrm{eV}$ disturbance of the electrochemical potential or huge voltage gradients of $10000000 \mathrm{~V} / \mathrm{cm}$. Therefore the temperature gradients here can be regarded as a much weaker disturbance. 


\section{Bibliography}

1. Seebeck, T. J. Polarisation der Metalle und Erze durch Temperaturdifferenz. Abhandlungen der Königlich Preußischen Akademie der Wissenschaften zu Berlin, 265-375 (1822) (cited on page 1).

2. Peltier, J. C. A. Nouvelle Expériences sur la Caloricité des courans électriques. Annales de Chimie et de Physique 56, 371-386 (1834) (cited on page 1).

3. Thomson, W. On the Dynamical Theory of Heat, Part VI. Thermo-electric Currents. Transactions of the Royal Society of Edinburgh XXI (1854) (cited on page 1).

4. Johnson, M. \& Silsbee, R. H. Thermodynamic analysis of interfacial transport and of the thermomagnetoelectric system. Phys. Rev. B 35, 4959-4972 (1987) (cited on pages 1, 24).

5. Bauer, G. E. W., MacDonald, A. H. \& Maekawa, S. Spin Caloritronics. Solid State Commun. 150, 459-460 (2010) (cited on pages 1, 24, 35).

6. Shi, J., Yu, R. C., Parkin, S. S. P. \& Salamon, M. B. Magnetothermopower of Co/Cu multilayers. J. Appl. Phys. 73, 5524 (1993) (cited on pages 1, 12, $35)$.

7. Gravier, L., Fábián, A., Rudolf, A., Cachin, A., Hjort, K. \& Ansermet, J.-P. Thermopower measurement of single isolated metallic nanostructures. Meas. Sci. Technol. 15, 420-424 (2004) (cited on pages 1, 12, 36).

8. Gravier, L., Serrano-Guisan, S., Reuse, F. \& Ansermet, J.-P. Thermodynamic description of heat and spin transport in magnetic nanostructures. Phys. Rev. $B$ 73, 024419 (2006) (cited on pages 1, 12, 23, 35).

9. Gravier, L., Serrano-Guisan, S., Reuse, F. \& Ansermet, J.-P. Spin-dependent Peltier effect of perpendicular currents in multilayered nanowires. Phys. Rev. $B$ 73, 052410 (2006) (cited on pages 1, 12).

10. Uchida, K., Takahashi, S., Harii, K., Ieda, J., Koshibae, W., Ando, K., Maekawa, S. \& Saitoh, E. Observation of the spin Seebeck effect. Nature 455, 778-781 (2008) (cited on pages 1, 10, 23, 35).

11. Bauer, G. E. W., Saitoh, E. \& van Wees, B. J. Spin caloritronics. Nature Mater. 11, 391-399 (2012) (cited on pages 2, 9-11, 35, 49).

12. Münzenberg, M. \& Moodera, J. S. Taking advantage of nature for a greener nonvolatile memory. Physics 3, 19 (2010) (cited on page 2). 
13. Brataas, A., Kent, A. D. \& Ohno, H. Current-induced torques in magnetic materials. Nature Mater. 11, 372-381 (2012) (cited on pages 2, 20).

14. Jansen, R. Silicon spintronics. Nature Mater. 11, 400-408 (2012) (cited on pages 2,15$)$.

15. Ikeda, S., Hayakawa, J., Ashizawa, Y., Lee, Y. M., Miura, K., Hasegawa, H., Tsunoda, M., Matsukura, F. \& Ohno, H. Tunnel magnetoresistance of 604\% at $300 \mathrm{~K}$ by suppression of Ta diffusion in $\mathrm{CoFeB} / \mathrm{MgO} / \mathrm{CoFeB}$ pseudo-spinvalves annealed at high temperature. Appl. Phys. Lett. 93, 082508 (2008) (cited on pages $2,26,50,59$ ).

16. Yuasa, S. \& Djayaprawira, D. D. Giant tunnel magnetoresistance in magnetic tunnel junctions with a crystalline $\mathrm{MgO}\left(\begin{array}{lll}0 & 0 & 1\end{array}\right)$ barrier. J. Phys. D: Appl. Phys. 40, R337-R354 (2007) (cited on pages 2, 49, 68).

17. Georg-August University School of Science. Doctoral Degree Regulations (RerNat-O) 2012 (cited on pages 3, 103).

18. Callen, H. B. Thermodynamics: An introduction to the physical theories of equilibrium thermostatics and irreversible thermodynamics 283-308 (John Wiley \& Sons Inc., New York, 1960) (cited on pages 3, 6-8, 39).

19. Ashcroft, N. W. \& Mermin, N. D. Solid State Physics (Thomson Learning, Inc., 1976) (cited on pages 4, 5, 7).

20. Chen, G. Nanoscale Energy Transport and Conversion (Oxford University Press, 2005) (cited on pages 4, 79, 80, 82, 83).

21. Sivan, U. \& Imry, Y. Multichannel Landauer formula for thermoelectric transport with application to thermopower near the mobility edge. Phys. Rev. B 33, 551-558 (1986) (cited on pages 4, 26, 39).

22. Ouyang, Y. \& Guo, J. A theoretical study on thermoelectric properties of graphene nanoribbons. Appl. Phys. Lett. 94, 263107 (2009) (cited on pages 4, $26,40)$.

23. Slachter, A., Bakker, F. L., Adam, J.-P. \& van Wees, B. J. Thermally driven spin injection from a ferromagnet into a non-magnetic metal. Nature Phys. 6, 879-882 (2010) (cited on pages 10, 12, 13, 23, 36).

24. Flipse, J., Bakker, F. L., Slachter, A., Dejene, F. K. \& van Wees, B. J. Direct observation of the spin-dependent Peltier effect. Nature Nanotech. 7, 166-168 (2012) (cited on pages 10, 12-14).

25. Uchida, K., Xiao, J., Adachi, H., Ohe, J., Takahashi, S., Ieda, J., Ota, T., Kajiwara, Y., Umezawa, H., Kawai, H., Bauer, G. E. W., Maekawa, S. \& Saitoh, E. Spin Seebeck insulator. Nature Mater. 9, 894-897 (2010) (cited on pages $10,23,35)$. 
26. Jaworski, C. M., Yang, J., Mack, S., Awschalom, D. D., Heremans, J. P. \& Myers, R. C. Observation of the spin-Seebeck effect in a ferromagnetic semiconductor. Nature Mater. 9, 898-903 (2010) (cited on pages 10, 23, 35).

27. Uchida, K.-i., Adachi, H., Ota, T., Nakayama, H., Maekawa, S. \& Saitoh, E. Observation of longitudinal spin-Seebeck effect in magnetic insulators. Appl. Phys. Lett. 97, 172505 (2010) (cited on page 10).

28. Avery, A. D., Pufall, M. R. \& Zink, B. L. Observation of the Planar Nernst Effect in Permalloy and Nickel Thin Films with In-Plane Thermal Gradients. Phys. Rev. Lett. 109, 196602 (2012) (cited on pages 10, 36).

29. Huang, S. Y., Fan, X., Qu, D., Chen, Y. P., Wang, W. G., Wu, J., Chen, T. Y., Xiao, J. Q. \& Chien, C. L. Transport Magnetic Proximity Effects in Platinum. Phys. Rev. Lett. 109, 107204 (2012) (cited on pages 11, 36).

30. Lu, Y. M., Choi, Y., Ortega, C. M., Cheng, X. M., Cai, J. W., Huang, S. Y., Sun, L. \& Chien, C. L. Pt Magnetic Polarization on $\mathrm{Y}_{3} \mathrm{Fe}_{5} \mathrm{O}_{12}$ and Magnetotransport Characteristics. Phys. Rev. Lett. 110, 147207 (2013) (cited on pages 11,36$)$.

31. Qu, D., Huang, S. Y., Hu, J., Wu, R. \& Chien, C. L. Intrinsic Spin Seebeck Effect in Au/YIG. Phys. Rev. Lett. 110, 067206 (2013) (cited on pages 11, $36)$.

32. Geprägs, S., Meyer, S., Altmannshofer, S., Opel, M., Wilhelm, F., Rogalev, A., Gross, R. \& Goennenwein, S. T. B. Investigation of induced Pt magnetic polarization in Pt/ $\mathrm{Y}_{3} \mathrm{Fe}_{5} \mathrm{O}_{12}$ bilayers. Appl. Phys. Lett. 101, 262407 (2012) (cited on pages 11,36).

33. Meier, D., Kuschel, T., Shen, L., Gupta, A., Kikkawa, T., Uchida, K., Saitoh, E., Schmalhorst, J.-M. \& Reiss, G. Thermally driven spin and charge currents in thin $\mathrm{NiFe}_{2} \mathrm{O}_{4} / \mathrm{Pt}$ films. Phys. Rev. B 87, 054421 (2013) (cited on pages 11, $12,36)$.

34. Bakker, F., Slachter, A., Adam, J.-P. \& van Wees, B. Interplay of Peltier and Seebeck Effects in Nanoscale Nonlocal Spin Valves. Phys. Rev. Lett. 105, 136601 (2010) (cited on pages 12, 36).

35. Le Breton, J.-C., Sharma, S., Saito, H., Yuasa, S. \& Jansen, R. Thermal spin current from a ferromagnet to silicon by Seebeck spin tunnelling. Nature 475, 82-85 (2011) (cited on pages 14, 15, 31, 36, 49).

36. Czerner, M., Bachmann, M. \& Heiliger, C. Spin caloritronics in magnetic tunnel junctions: Ab initio studies. Phys. Rev. B 83, 132405 (2011) (cited on pages $16,24,36,39,40,50)$. 
37. Lin, W., Hehn, M., Chaput, L., Negulescu, B., Andrieu, S., Montaigne, F. \& Mangin, S. Giant thermoelectric effect in $\mathrm{Al}_{2} \mathrm{O}_{3}$ magnetic tunnel junctions. Nature Commun. 3, 744 (2012) (cited on pages 16-19, 36, 39, 43, 50, 75, 78).

38. Butler, W. H., Zhang, X. G., Schulthess, T. C. \& MacLaren, J. M. Spindependent tunneling conductance of $\mathrm{Fe} / \mathrm{MgO} / \mathrm{Fe}$ sandwiches. Phys. Rev. B 63, 054416 (2001) (cited on pages 18, 50, 59).

39. Liebing, N., Serrano-Guisan, S., Rott, K., Reiss, G., Langer, J., Ocker, B. \& Schumacher, H. W. Determination of spin-dependent Seebeck coefficients of $\mathrm{CoFeB} / \mathrm{MgO} / \mathrm{CoFeB}$ magnetic tunnel junction nanopillars. J. Appl. Phys. 111, 07C520 (2012) (cited on pages 18, 19, 38, 41, 86).

40. Liebing, N., Serrano-Guisan, S., Rott, K., Reiss, G., Langer, J., Ocker, B. \& Schumacher, H. W. Tunneling Magnetothermopower in Magnetic Tunnel Junction Nanopillars. Phys. Rev. Lett. 107, 177201 (2011) (cited on pages 18, $31,36,50,86)$.

41. Liebing, N., Serrano-Guisan, S., Krzysteczko, P., Rott, K., Reiss, G., Langer, J., Ocker, B. \& Schumacher, H. W. Tunneling magneto thermocurrent in $\mathrm{CoFeB} / \mathrm{MgO} / \mathrm{CoFeB}$ based magnetic tunnel junctions. Appl. Phys. Lett. 102, 242413 (2013) (cited on pages 18, 19, 43, 75, 86).

42. Hatami, M., Bauer, G. E. W., Zhang, Q. \& Kelly, P. J. Thermal Spin-Transfer Torque in Magnetoelectronic Devices. Phys. Rev. Lett. 99, 066603 (2007) (cited on page 19).

43. Slonczewski, J. C. Initiation of spin-transfer torque by thermal transport from magnons. Phys. Rev. B 82, 054403 (2010) (cited on pages 20, 50).

44. Jia, X., Xia, K. \& Bauer, G. Thermal Spin Transfer in Fe-MgO-Fe Tunnel Junctions. Phys. Rev. Lett. 107, 176603 (2011) (cited on pages 20, 21, 36, $50,55,57,86)$.

45. Müller, G. M., Walowski, J., Djordjevic, M., Miao, G.-X., Gupta, A., Ramos, A. V., Gehrke, K., Moshnyaga, V., Samwer, K., Schmalhorst, J., Thomas, A., Hütten, A., Reiss, G., Moodera, J. S. \& Münzenberg, M. Spin polarization in half-metals probed by femtosecond spin excitation. Nature Mater. 8, 56-61 (2009) (cited on pages 24, 69).

46. Heiliger, C., Czerner, M., Yavorsky, B. Y., Mertig, I. \& Stiles, M. D. Implementation of a nonequilibrium Green's function method to calculate spin-transfer torque. J. Appl. Phys. 103, 07A709 (2008) (cited on pages 26, 54).

47. Lee, S.-M., Cahill, D. G. \& Allen, T. H. Thermal conductivity of sputtered oxide films. Phys. Rev. B 52, 253-257 (1995) (cited on pages 27, 33, 38, 39, $89)$. 
48. Thomas, A., Drewello, V., Schäfers, M., Weddemann, A., Reiss, G., Eilers, G., Münzenberg, M., Thiel, K. \& Seibt, M. Direct imaging of the structural change generated by dielectric breakdown in $\mathrm{MgO}$ based magnetic tunnel junctions. Appl. Phys. Lett. 93, 152508 (2008) (cited on pages 29, 42).

49. Walter, M., Walowski, J., Zbarsky, V., Münzenberg, M., Schäfers, M., Ebke, D., Reiss, G., Thomas, A., Peretzki, P., Seibt, M., Moodera, J. S., Czerner, M., Bachmann, M. \& Heiliger, C. Seebeck effect in magnetic tunnel junctions. Nature Mater. 10, 742-746 (2011) (cited on pages 36-40, 50, 54, 56, 57, 73, 103).

50. Leutenantsmeyer, J. C., Walter, M., Zbarsky, V., Münzenberg, M., Gareev, R., Rott, K., Thomas, A., Reiss, G., Peretzki, P., Schuhmann, H., Seibt, M., Czerner, M. \& Heiliger, C. Parameter space for thermal spin-transfer torque. SPIN 03, 1350002 (2013) (cited on pages 36, 39, 103).

51. Kikkawa, T., Uchida, K., Shiomi, Y., Qiu, Z., Hou, D., Tian, D., Nakayama, H., Jin, X.-F. \& Saitoh, E. Longitudinal Spin Seebeck Effect Free from the Proximity Nernst Effect. Phys. Rev. Lett. 110, 067207 (2013) (cited on page 36).

52. Heiliger, C., Franz, C. \& Czerner, M. Ab initio studies of the tunneling magneto-Seebeck effect: Influence of magnetic material. Phys. Rev. B 87, 224412 (2013) (cited on pages 36, 59-61, 68).

53. Papusoi, C., Sousa, R., Herault, J., Prejbeanu, I. L. \& Dieny, B. Probing fast heating in magnetic tunnel junction structures with exchange bias. New J. Phys. 10, 103006 (2008) (cited on page 38).

54. Beecher, S., Dinwiddie, R., Abeel, A. \& Lowden, R. The thermal conductivity of silicon nitride with molybdenum disilicide additions (ed Tong, T. W.) (Technomic Publishing Company, 1994) (cited on page 38).

55. Zink, B. L., Revaz, B., Cherry, J. J. \& Hellman, F. Measurement of thermal conductivity of thin films with a Si-N membrane-based microcalorimeter. Rev. Sci. Instrum. 76, 024901 (2005) (cited on page 38).

56. Zhang, Q. G., Cao, B. Y., Zhang, X., Fujii, M. \& Takahashi, K. Influence of grain boundary scattering on the electrical and thermal conductivities of polycrystalline gold nanofilms. Phys. Rev. B 74, 134109 (2006) (cited on page 38).

57. COMSOL Multiphysics with Heat Transfer Module (cited on pages 39, 47).

58. Büttiker, M., Imry, Y., Landauer, R. \& Pinhas, S. Generalized many-channel conductance formula with application to small rings. Phys. Rev. B 31, $6207-$ 6215 (1985) (cited on page 39). 
59. Slonczewski, J. C. Current-driven excitation of magnetic multilayers. J. Magn. Magn. Mater. 159, L1-L7 (1996) (cited on page 50).

60. Berger, L. Emission of spin waves by a magnetic multilayer traversed by a current. Phys. Rev. B 54, 9353-9358 (1996) (cited on page 50).

61. Parkin, S. S. P., Kaiser, C., Panchula, A., Rice, P. M., Hughes, B., Samant, M. \& Yang, S. Giant tunnelling magnetoresistance at room temperature with MgO (100) tunnel barriers. Nature Mater. 3, 862-867 (2004) (cited on page 50).

62. Yuasa, S., Nagahama, T., Fukushima, A., Suzuki, Y. \& Ando, K. Giant roomtemperature magnetoresistance in single-crystal $\mathrm{Fe} / \mathrm{MgO} / \mathrm{Fe}$ magnetic tunnel junctions. Nature Mater. 3, 868-871 (2004) (cited on page 50).

63. Miao, G.-X., Münzenberg, M. \& Moodera, J. S. Tunneling path toward spintronics. Rep. Prog. Phys. 74, 036501 (2011) (cited on page 50).

64. Yuasa, S., Suzuki, Y., Katayama, T. \& Ando, K. Characterization of growth and crystallization processes in $\mathrm{CoFeB} / \mathrm{MgO} / \mathrm{CoFeB}$ magnetic tunnel junction structure by reflective high-energy electron diffraction. Appl. Phys. Lett. 87, 242503 (2005) (cited on page 50).

65. Eilers, G., Ulrichs, H., Münzenberg, M., Thomas, A., Thiel, K. \& Seibt, M. Long-range order on the atomic scale induced at $\mathrm{CoFeB} / \mathrm{MgO}$ interfaces. $J$. Appl. Phys. 105, 073701 (2009) (cited on page 50).

66. Isogami, S., Tsunoda, M., Komagaki, K., Sunaga, K., Uehara, Y., Sato, M., Miyajima, T. \& Takahashi, M. In situ heat treatment of ultrathin MgO layer for giant magnetoresistance ratio with low resistance area product in $\mathrm{CoFeB} / \mathrm{MgO} / \mathrm{CoFeB}$ magnetic tunnel junctions. Appl. Phys. Lett. 93, 192109 (2008) (cited on page 51).

67. Kurt, H., Oguz, K., Niizeki, T. \& Coey, J. M. D. Giant tunneling magnetoresistance with electron beam evaporated $\mathrm{MgO}$ barrier and $\mathrm{CoFeB}$ electrodes. J. Appl. Phys. 107, 083920 (2010) (cited on page 51).

68. Duda, R. O. \& Hart, P. E. Use of the Hough transformation to detect lines and curves in pictures. Commun. ACM 15, 11-15 (1972) (cited on page 52).

69. Ma, Q. L., Kubota, T., Mizukami, S., Zhang, X. M., Naganuma, H., Oogane, M., Ando, Y. \& Miyazaki, T. Magnetoresistance effect in $\mathrm{L}_{0}-\mathrm{MnGa} / \mathrm{MgO} /$ CoFeB perpendicular magnetic tunnel junctions with Co interlayer. Appl. Phys. Lett. 101, 032402 (2012) (cited on page 53).

70. Heiliger, C. \& Stiles, M. D. Ab Initio Studies of the Spin-Transfer Torque in Magnetic Tunnel Junctions. Phys. Rev. Lett. 100, 186805 (2008) (cited on pages 53-55). 
71. Ikeda, S., Miura, K., Yamamoto, H., Mizunuma, K., Gan, H. D., Endo, M., Kanai, S., Hayakawa, J., Matsukura, F. \& Ohno, H. A perpendicular-anisotropy CoFeB-MgO magnetic tunnel junction. Nature Mater. 9, 721-724 (2010) (cited on pages 53, 54).

72. Khalili Amiri, P., Zeng, Z. M., Langer, J., Zhao, H., Rowlands, G., Chen, Y.-J., Krivorotov, I. N., Wang, J.-P., Jiang, H. W., Katine, J. A., Huai, Y., Galatsis, K. \& Wang, K. L. Switching current reduction using perpendicular anisotropy in $\mathrm{CoFeB}-\mathrm{MgO}$ magnetic tunnel junctions. Appl. Phys. Lett. 98, 112507 (2011) (cited on page 53).

73. Sun, J. Z. Spin-current interaction with a monodomain magnetic body: A model study. Phys. Rev. B 62, 570-578 (2000) (cited on page 53).

74. Hayakawa, J., Ikeda, S., Lee, Y. M., Sasaki, R., Meguro, T., Matsukura, F., Takahashi, H. \& Ohno, H. Current-Driven Magnetization Switching in $\mathrm{CoFeB} / \mathrm{MgO} / \mathrm{CoFeB}$ Magnetic Tunnel Junctions. Jap. J. Appl. Phys. 44, L1267-L1270 (2005) (cited on page 54).

75. Matsumoto, R., Fukushima, A., Yakushiji, K., Yakata, S., Nagahama, T., Kubota, H., Katayama, T., Suzuki, Y., Ando, K., Yuasa, S., Georges, B., Cros, V., Grollier, J. \& Fert, A. Spin-torque-induced switching and precession in fully epitaxial $\mathrm{Fe} / \mathrm{MgO} / \mathrm{Fe}$ magnetic tunnel junctions. Phys. Rev. B 80, 174405 (2009) (cited on page 54).

76. Johnson, P. B. \& Christy, R. W. Optical Constants of the Noble Metals. Phys. Rev. B 6, 4370-4379 (1972) (cited on page 57).

77. Handbook of Optical Constants of Solids (ed Palik, E. D.) (Academic Press, Boston, 1985) (cited on page 57).

78. Mathon, J. \& Umerski, A. Theory of tunneling magnetoresistance of an epitaxial Fe/MgO/Fe(001) junction. Phys. Rev. B 63, 220403 (2001) (cited on page 59).

79. Eggebrecht, T. Magneto-Seebeck-Effekt in Tunnelelementen mit senkrechter magnetischer Anisotropie Master's thesis (Georg-August-Universität Göttingen, 2013) (cited on pages 62, 72, 73, 75, 77, 78).

80. Mann, A., Walowski, J., Münzenberg, M., Maat, S., Carey, M. J., Childress, J. R., Mewes, C., Ebke, D., Drewello, V., Reiss, G. \& Thomas, A. Insights into Ultrafast Demagnetization in Pseudogap Half-Metals. Phys. Rev. X 2, 041008 (2012) (cited on page 69).

81. Mazierska, J., Ledenyov, D., Jacob, M. V. \& Krupka, J. Precise microwave characterization of $\mathrm{MgO}$ substrates for HTS circuits with superconducting post dielectric resonator. Supercond. Sci. Technol. 18, 18-23 (2005) (cited on page 72 ). 
82. Bosu, S., Sakuraba, Y., Uchida, K., Saito, K., Ota, T., Saitoh, E. \& Takanashi, K. Spin Seebeck effect in thin films of the Heusler compound $\mathrm{Co}_{2} \mathrm{MnSi}$. Phys. Rev. B 83, 224401 (2011) (cited on page 79).

83. Simmons, J. G. Generalized Formula for the Electric Tunnel Effect between Similar Electrodes Separated by a Thin Insulating Film. J. Appl. Phys. 34, 1793-1803 (1963) (cited on page 80).

84. Walter, M. Untersuchung der Transporteigenschaften von $\mathrm{CoFeB} / \mathrm{MgO} / \mathrm{CoFeB}$ Tunnelmagnetowiderstandselementen diploma thesis (Georg-August-Universität Göttingen, 2009) (cited on pages 81, 83).

85. Costescu, R. M., Wall, M. A. \& Cahill, D. G. Thermal conductance of epitaxial interfaces. Phys. Rev. B 67, 054302 (2003) (cited on pages 82, 83).

86. Costescu, R. M., Cahill, D. G., Fabreguette, F. H., Sechrist, Z. A. \& George, S. M. Ultra-Low Thermal Conductivity in W/ $/ \mathrm{Al}_{2} \mathrm{O}_{3}$ Nanolaminates. Science 303, 989-990 (2004) (cited on page 83).

87. Cahill, D. G. Analysis of heat flow in layered structures for time-domain thermoreflectance. Rev. Sci. Instrum. 75, 5119-5122 (2004) (cited on page 89).

88. Heiliger, C., Zahn, P., Yavorsky, B. Y. \& Mertig, I. Interface structure and bias dependence of $\mathrm{Fe} / \mathrm{MgO} / \mathrm{Fe}$ tunnel junctions: Ab initio calculations. Phys. Rev. B 73, 214441 (2006) (cited on page 94).

89. Boehnke, A., Walter, M., Roschewsky, N., Eggebrecht, T., Drewello, V., Rott, K., Münzenberg, M., Thomas, A. \& Reiss, G. Time-resolved measurement of the tunnel magneto-Seebeck effect in a single magnetic tunnel junction. Rev. Sci. Instrum. 84, 063905 (2013) (cited on page 103). 


\section{Author contributions}

The chapters 2, 3 and 4 present reprints of manuscripts published in peer-reviewed scientific journals. As required by the regulations of the PhD program of the GeorgAugust-University School of Science ${ }^{17}$, this section comprises the contributions of the respective authors to the following manuscripts:

- Seebeck effect in magnetic tunnel junctions. Nature Materials 10, 742-746 (2011) (Ref. [49])

- Time-resolved measurement of the tunnel magneto-Seebeck effect in a single magnetic tunnel junction. Review of Scientific Instruments 84, 063905 (2013) (Ref. [89])

- Parameter space for thermal spin transfer torque. SPIN 3, 1350002 (2013) (Ref. [50])

The author contributions of the first manuscript "Seebeck effect in magnetic tunnel junctions" are already contained in section 2.3, but shall be repeated here - with author names written in full - for the sake of completeness: The author of this thesis (in the following: the author) and Jakob Walowski carried out experiments; the author, Vladyslav Zbarsky, Markus Schäfers and Daniel Ebke characterized and prepared the TMR devices; Patrick Peretzki and Michael Seibt carried out the high-resolution TEM; the author, Jakob Walowski and Markus Münzenberg analyzed the data; the author carried out the COMSOL calculations; Michael Czerner, Michael Bachmann and Christian Heiliger did the ab initio transport calculations; Andy Thomas and Markus Münzenberg designed the research approach; Christian Heiliger, Markus Münzenberg and Andy Thomas wrote the manuscript and developed the model; the author, Jagadeesh S. Moodera, Andy Thomas, Markus Münzenberg and Christian Heiliger contributed to the development of the experiments; Günter Reiss, Jagadeesh S. Moodera, Andy Thomas, Markus Münzenberg, Christian Heiliger and all authors discussed the experiments and the manuscript.

The manuscript "Time-resolved measurement of the tunnel magneto-Seebeck effect in a single magnetic tunnel junction" was written by Alexander Böhnke and the author. The samples were prepared by Karsten Rott and Volker Drewello. The experimental setup was optimized by Niklas Roschewsky, Tim Eggebrecht and the author. The COMSOL simulations presented in the manuscript were carried out by the author. The experiments were carried out by Alexander Böhnke, Tim Eggebrecht and the author. Alexander Böhnke, Markus Münzenberg and the author 
analyzed the data. The model circuit presented in this manuscript was developed by Alexander Böhnke, Markus Münzenberg, Niklas Roschewsky, Tim Eggebrecht and the author. Markus Münzenberg, Andy Thomas and Günter Reiss designed the research approach. All authors discussed the experiments, data and manuscript.

The manuscript "Parameter space for thermal spin transfer torque" was written by Johannes Christian Leutenantsmeyer, Patrick Peretzki, Christian Heiliger and the author. The samples presented in this paper were prepared by Johannes Christian Leutenantsmeyer. The optimization of growth parameters for the $\mathrm{MgO}$ barrier was done by Johannes Christian Leutenantsmeyer and the author. Vladyslav Zbarsky and Rashid Gareev contributed to the development of MTJs with perpendicular magnetic anisotropy. Karsten Rott and Johannes Christian Leutenantsmeyer patterned the samples. Patrick Peretzki, Henning Schuhmann and Michael Seibt did the quantitative HRTEM analysis. Spin-transfer torque measurements were performed and analyzed by Johannes Christian Leutenantsmeyer, Karsten Rott and the author. The ab initio calculations were done by Michael Czerner and Christian Heiliger. The author carried out the COMSOL simulations. Markus Münzenberg, Andy Thomas, Günter Reiss and Christian Heiliger designed the research approach and directed research. All authors discussed the experiments, calculations and simulations, as well as the manuscript. 


\section{Acknowledgements}

The thesis would not have been possible in this form without the support of other people. At this point I would like to thank first of all Prof. Dr. Markus Münzenberg for supervising this thesis and the trust he has shown to me. Numerous, fruitful discussions and advices as well as the support of own ideas and the organization of social activities with the research group have created a great working atmosphere over the last years. His commitment to physics and his research group are exemplary. Further, I would like to thank Prof. Dr. Christian Jooß for accepting to review this thesis.

The work would not have been as pleasant and entertaining over the last years without the other members of the "Spin-group". Especially, I would like to say thank you to Gerrit Eilers, Jakob Walowski, Benjamin Lenk, Kai Ubbo Ubben, Vladyslav Zbarsky, Johannes Christian Leutenantsmeyer, Martin Lüttich, Anissa Zeghuzi, Mirco Marahrens, Niklas Roschewsky, Tim Eggebrecht and Marius Milnikel, but also to those who are not mentioned by name. It was a great time!

I also would like to acknowledge the support by the people from the collaborations with Giessen, Bielefeld and the IV. Physics Institute in Göttingen, in particular Alexander Böhnke, Henning Schuhmann, Patrick Peretzki, Dr. Andy Thomas, Prof. Dr. Günter Reiss and Prof. Dr. Christian Heiliger.

The experiments were enabled by the financial support of Prof. Dr. Konrad Samwer and the technical support of Carsten Mahn, Uta Filippich, Katrin Gehrke, the workshop around Hartmut Eichenberg, and the electrical workshop. Especially Carsten, Uta, and Katrin were always available and offered a helping hand to realize the ideas that occured in the lab.

For careful proof-reading of the thesis, I would like to express my gratitude to Gerrit Eilers, Jakob Walowski, Alexander Böhnke, Amber Smith and Katrin von der Ehe.

The years at university would not be as unforgettable without my friends, in particular, Murat Sivis, Kai Ubbo Ubben, Benjamin Lenk, Georg Herink, Björn Hoffmann, Björn Enders, Steffen Ravekes, Michael Mell, Max-Julian Waack, Jakob Walowski and Gerrit Eilers. Thank you!

Last but not least, I am deeply grateful for the moral support from Katrin von der Ehe, my parents Reinhold 65 Silvia and my sister Marlena Walter. Thank you for always being there for me. 